\title{
Correctional Officer Opinions about Offenders with Mental Illness: The Relationships Among Opinions, Burnout, Emotional Intelligence, and Mental Health Training
}

Courtney Hull

cnh0008@mix.wvu.edu

Follow this and additional works at: https://researchrepository.wvu.edu/etd

Part of the Law Enforcement and Corrections Commons, and the Other Psychology Commons

\section{Recommended Citation}

Hull, Courtney, "Correctional Officer Opinions about Offenders with Mental Illness: The Relationships Among Opinions, Burnout, Emotional Intelligence, and Mental Health Training" (2021). Graduate Theses, Dissertations, and Problem Reports. 7808.

https://researchrepository.wvu.edu/etd/7808

This Dissertation is protected by copyright and/or related rights. It has been brought to you by the The Research Repository @ WVU with permission from the rights-holder(s). You are free to use this Dissertation in any way that is permitted by the copyright and related rights legislation that applies to your use. For other uses you must obtain permission from the rights-holder(s) directly, unless additional rights are indicated by a Creative Commons license in the record and/ or on the work itself. This Dissertation has been accepted for inclusion in WVU Graduate Theses, Dissertations, and Problem Reports collection by an authorized administrator of The Research Repository @ WVU.

For more information, please contact researchrepository@mail.wvu.edu. 
Correctional Officer Opinions about Offenders with Mental Illness: The Relationships Among Opinions, Burnout, Emotional Intelligence, and Mental Health Training

\author{
Courtney Hull, M.S. \\ Dissertation submitted \\ to the College of Education and Human Services \\ at West Virginia University
}

in partial fulfillment of the requirements for the degree of

Doctor of Philosophy in

Counseling Psychology

Department of Counseling \& Learning Sciences

Jeffrey Daniels, Ph.D., Co-Chair
Christine J. Schimmel, Ed.D., Co-Chair

Lisa F. Platt, Ph.D.

Edward Baker, Ph.D.

Morgantown, West Virginia

2020

Keywords: Correctional Officer, Opinions, Attitudes, Mental Illness, Burnout, Emotional Intelligence, Mental Health Training, Offender, Inmate

Copyright 2020 Courtney Hull 


\begin{abstract}
Rates of mental illness among incarcerated individuals in the United States are high and have been well documented. Correctional officers are on the front line of identifying symptoms and alerting mental health professionals to problems as they arise. Correctional officers often lack an understating of mental illness, feel ill-equipped to manage mental health crises, and experience high levels of job-related stress. The objective of this study was to better understand the relationship between correctional officers' emotional intelligence and opinions about mental illness, and to identify if burnout moderated the relationship between the variables. Additionally, the study sought to understand the relationship between mental health training and emotional intelligence, and to identify if mental health training moderated the relationship between emotional intelligence and opinions about mental illness. One hundred and five correctional officers completed a survey containing the Schutte Self-Report Emotional Intelligence Test, Opinions About Mental Illness Scale, Copenhagen Burnout Inventory, and a demographic questionnaire. Results of the study found that OMI does not significantly predict SSEIT. When accounting for OMI, CBI independently predicts SSEIT, indicating that as burnout increases, levels of emotional intelligence also increase. Burnout was not found to moderate the relationship between OMI and SSEIT. Further analyses indicated that mental health training does not significantly predict SSEIT, and that mental health training does not moderate the relationship between OMI and SSEIT. The results of this study have implications for understanding correctional officer experiences and providing them with resources to effectively work with inmates who suffer from mental illness.
\end{abstract}




\section{DEDICATION}

There have been many individuals throughout this journey who have given me the support and encouragement needed to complete this process. First and foremost, I would like to dedicate this dissertation to my loving parents and sister, who have made countless sacrifices, and who have been a constant and steady presence at every milestone I have achieved. None of this would be possible without your examples of hard work, perseverance, and humility.

I would also like to dedicate this work to my partner, who has offered unwavering love and support throughout this process. He has provided me with tremendous guidance during the creation of this work and has also challenged me to grow intellectually and personally as we have navigated the pursuit of doctoral degrees together. He uniquely balances me, and inspires me daily with his inquisitive nature, humor, and dedication to the field. I am truly blessed to walk through this life with him.

Lastly, I would like to dedicate this dissertation to my close friends, mentors, and cohort members, who have always believed in me, challenged me to become the best version of myself, and lifted me up in difficult times. I am eternally grateful for your patience and guidance. 


\section{ACKNOWLEDGEMENTS}

The graduate school and doctoral dissertation processes have been daunting, and I am thankful for the many people who have guided me along this journey. I would like to thank my dissertation chairs, Dr. Jeffrey Daniels and Dr. Christine Schimmel, who have helped me to successfully complete this work. I would have been lost without you, and I am grateful for your knowledge and guidance. Thank you for working together to continually move me forward, and for the wisdom and encouragement you imparted along the way.

I would also like to thank my committee members, Dr. Lisa Platt and Dr. Ed Baker. Dr. Platt was invaluable in developing this document. I am thankful for her support and availability, and for going above and beyond to ensure that I successfully completed this process. Dr. Baker has served as a mentor for many years and has been a valuable source of knowledge about the subject matter. I am thankful for the opportunities that he has provided me in the forensic field, and for the countless pieces of advice he has provided me.

There are many other staff members who have made obtaining this degree seamless and memorable, including Vicki Railing, Dr. James Bartee, and Dr. David Allen. I have felt welcomed since arriving at WVU and from them have gained vital knowledge and skills to ensure that I enter the workforce as a passionate and competent psychologist. 


\section{TABLE OF CONTENTS}

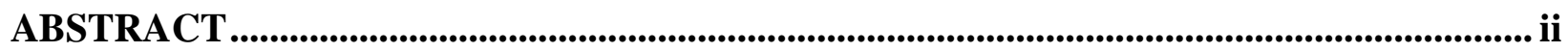

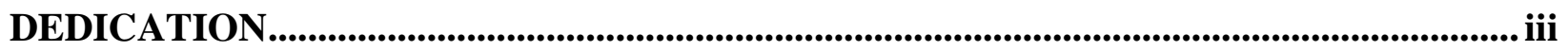

ACKNOWLEDGEMENTS _...............................................................................................

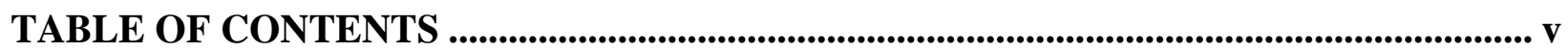

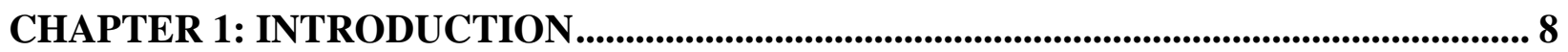

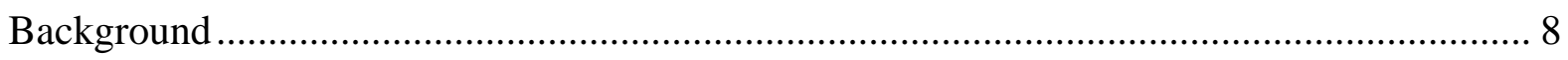

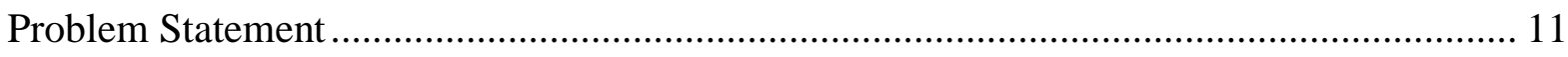

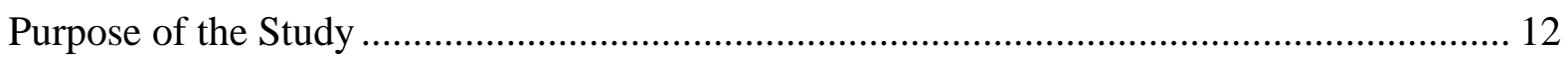

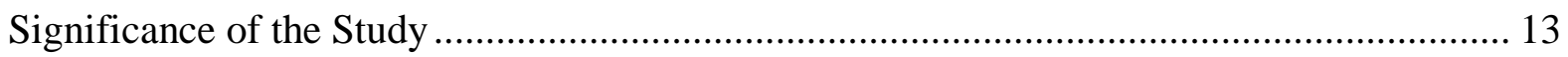

Research Questions and Hypotheses ............................................................................ 14

Definitions of Correctional Concepts ............................................................................. 16

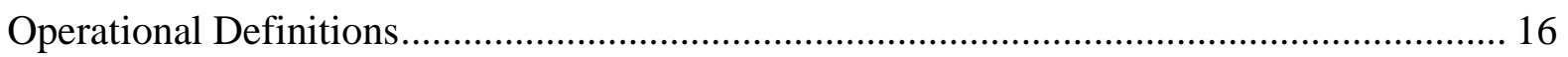

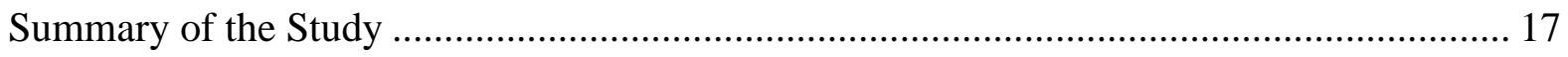

CHAPTER 2: REVIEW OF THE LITERATURE......................................................... 18

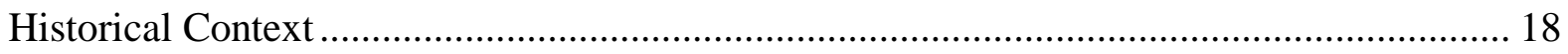

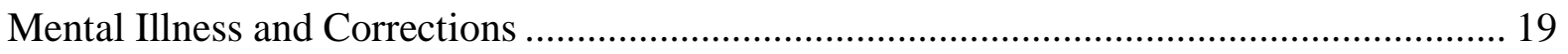

Mental Health Treatment in Corrections .......................................................................... 20

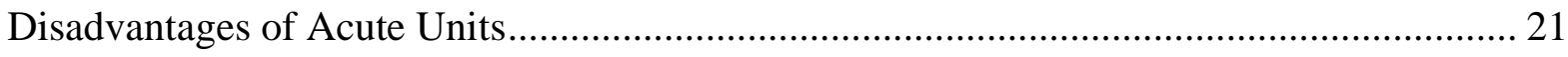

Advantages of Acute Units .......................................................................................... 22

Mental Health Professional and Correctional Officer Differences .......................................... 22

Correctional Officer Perceptions and Attitudes …………….............................................. 23

Correctional Officer Personality Traits......................................................................... 25

Correctional Officer Burnout ..................................................................................... 27

Mental Health Training in Corrections ....................................................................... 29

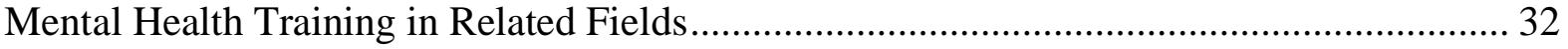

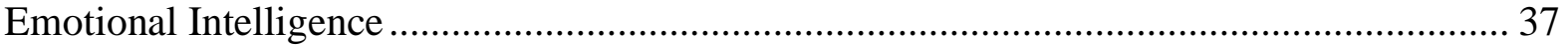

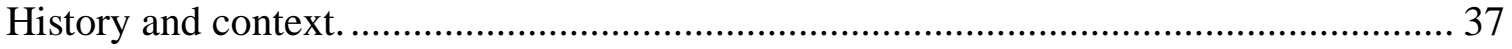

Conceptual framework.................................................................................. 39

Primary models.

Theoretical perspectives........................................................................................... 40

Measures and assessments. ........................................................................................ 41

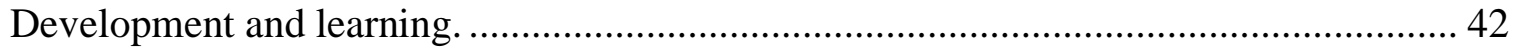




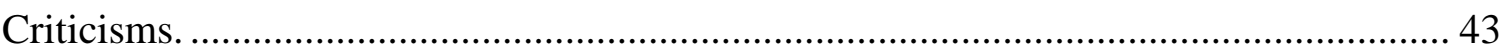

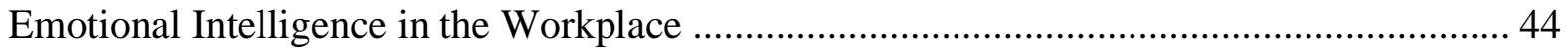

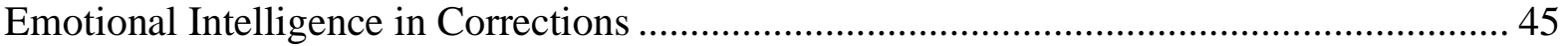

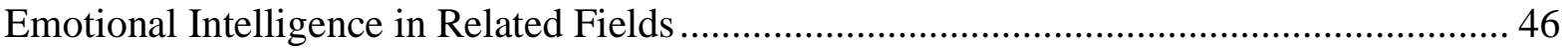

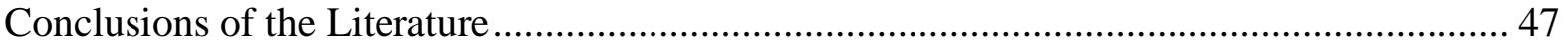

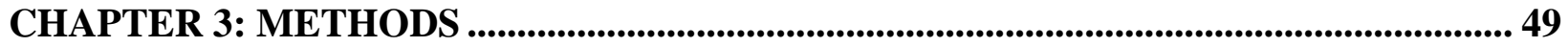

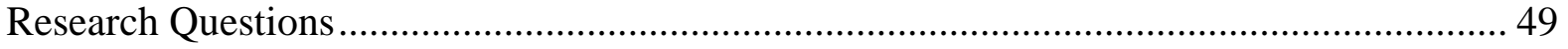

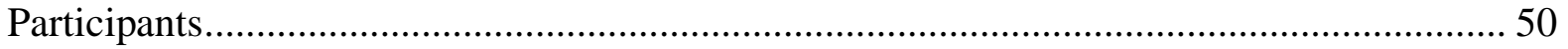

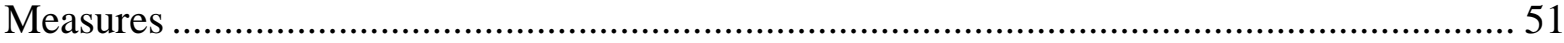

Opinions about Mental Illness Scale (OMI; Cohen, \& Struening, 1962)...................... 51

Schutte Self-Report Emotional Intelligence Test (SSEIT; Schutte, et al., 1998). ............ 53

Copenhagen Burnout Inventory (CBI; Kristensen, Borritz, Villadsen, \& Christensen,

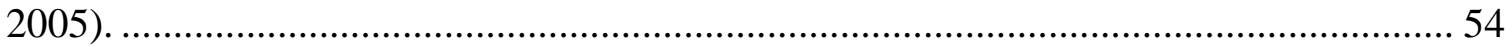

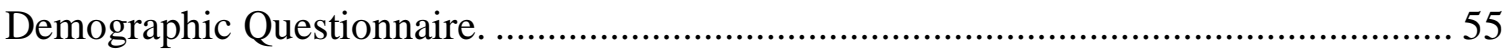

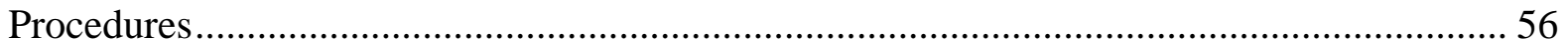

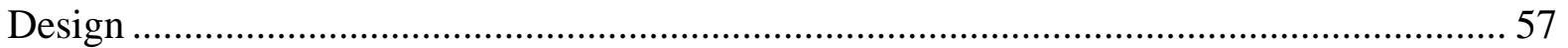

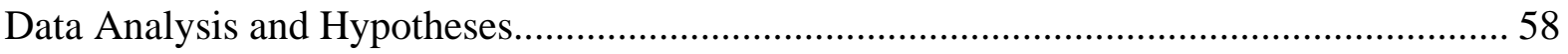

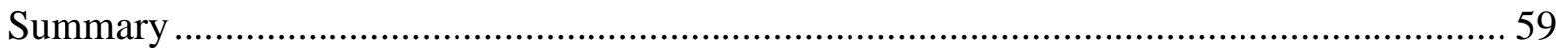

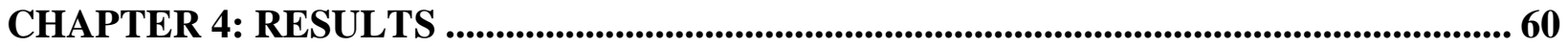

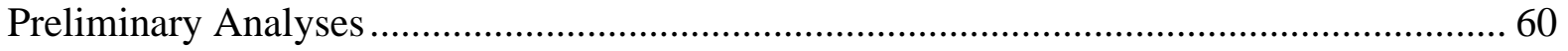

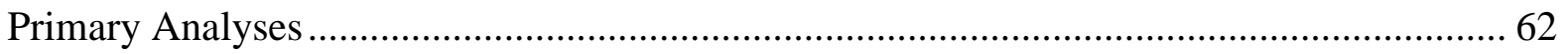

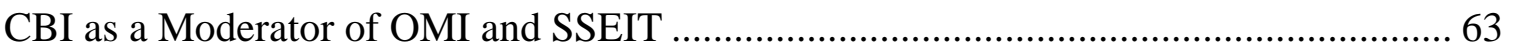

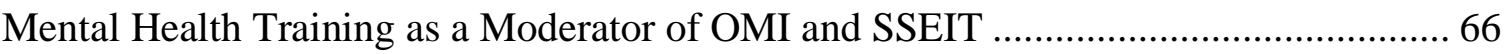

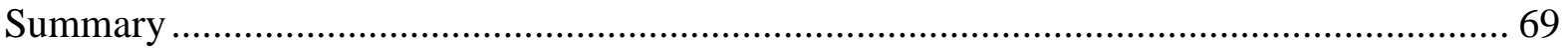

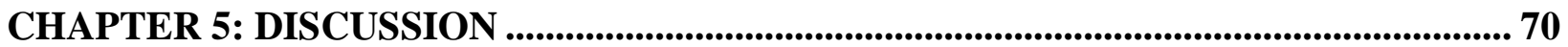

Discussion of Participant Demographics ....................................................................... 70

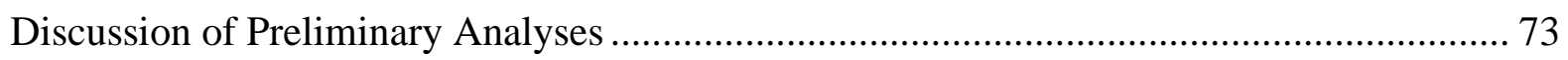

Discussion of CBI as a Moderator of OMI and SSEIT ….............................................. 75

Discussion of Mental Health Training as a Moderator of OMI and SSEIT ........................ 78

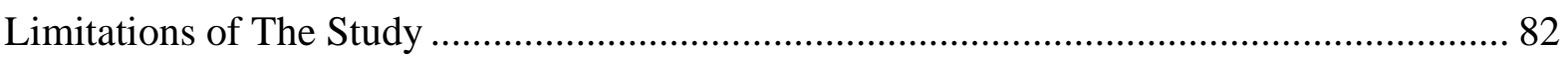

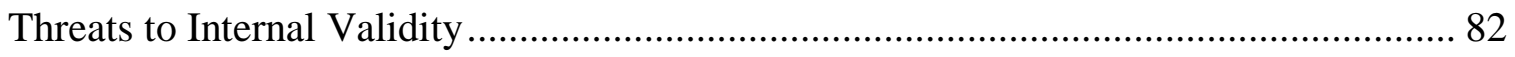

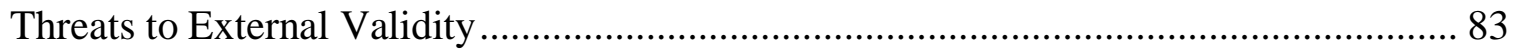

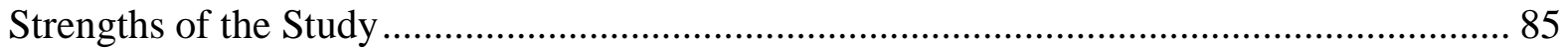

Implications for Counseling Psychology ..................................................................... 86

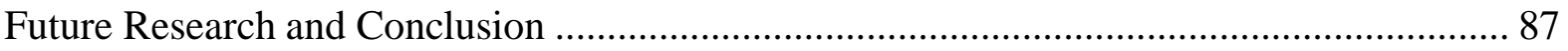




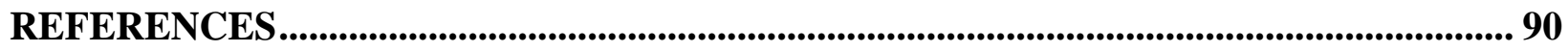

APPENDICES ……................................................................................................................... 114

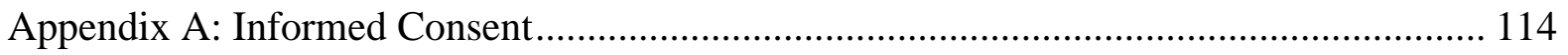

Appendix B: Participant Invitation Request (Social Media) ......................................... 117

Appendix C: Two Week Participation Invitation Follow-Up (Social Media) ..................... 119

Appendix D: Four Week Participation Invitation Follow-Up (Social Media) .................... 120

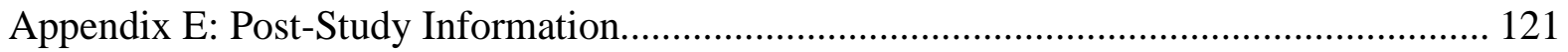

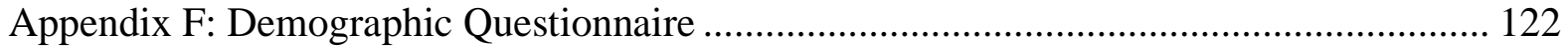

Appendix G: Opinions About Mental Illness Scales .................................................... 123

Appendix H: Schutte Self-Report Emotional Intelligence Test ....................................... 126

Appendix I: Copenhagen Burnout Inventory ............................................................. 129

Appendix J: Acknowledgement of Exemption ........................................................... 134

Appendix K: IRB Approval of Protocol Amendment …............................................... 136

Appendix L: Letter Requesting Access to the SSEIT ..................................................... 139

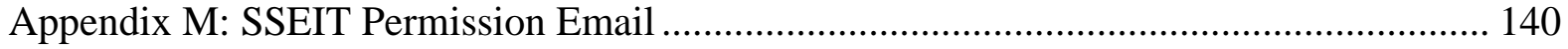

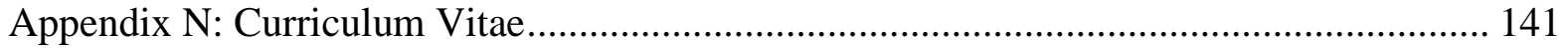




\section{CHAPTER 1: INTRODUCTION}

\section{Introduction and Rationale}

The prevalence of mental illness among incarcerated individuals in the United States has been well documented over the past twenty years, and numbers remain high (Aufderheide, 2014; Center, 2014; Glaze \& Herberman, 2012). Offenders with mental illness inevitably encounter correctional officers, the primary source of safety and security in prisons (Adams \& Ferrandino, 2008; Rhodes, 2004). Correctional officers frequently interact with mentally ill inmates and often play important roles in the treatment of offender mental illness, yet little is known about correctional officer attitudes toward and opinions of this segment of the prison population (Appelbaum, Hickey, \& Packer, 2001; Bureau of Labor Statistics, 2017; Dvoskin, \& Spiers, 2004; Galanek, 2015; Mauro, 2009; Sun, 2013). Further, there is limited research that explains individual correctional officer characteristics associated with positive attitudes (Callahan, 2004; Kropp, Cox, Roesch \& Eaves, 1989; Stys \& Brown, 2004).

The aim of this dissertation was to add to the existing literature regarding correctional officer opinions of offenders with mental illness. Further, it aimed to expand our knowledge concerning the relationship between correctional officer's opinions of mental illness, emotional intelligence, burnout, and mental health training. Such knowledge will aid mental health professionals and correctional facility administrators to work effectively as part of interdisciplinary teams on behalf of correctional officers, as well as mentally ill offenders. Additionally, it will aid in the development of training opportunities for officers.

\section{Background}

In the United States, approximately $20 \%$ of incarcerated individuals suffer from a serious mental illness, making jails and prisons the largest mental health institutions in the country 
(Kaeble, Glade, Tsoutis, \& Minton, 2016; Steadman, Osher, Robbins, Case, \& Samuels, 2009;

Torrey, Kennard, Eslinger, Lamb, \& Pavle, 2010; Torrey, et al., 2014). Because of the prevalence of mental illness in incarcerated populations, it is important to better understand the services with which affected individuals interact. One of the primary services in prisons is safety and security (Bureau of Labor Statistics, 2017; Liebling, Price \& Shefer, 2010). This role is fulfilled by correctional officers, who are tasked with ensuring the safety and security of facilities and managing the offenders that are housed therein (Adams \& Ferrandino, 2008; Galanek, 2015; Rhodes, 2004).

Limited research exists that examines correctional officer experiences within the context of their interactions with mentally ill offenders, attitudes toward mentally ill offenders, or their perceived ability to manage this unique population (Petracek, 2012; Skeem, Manchak, \& Peterson, 2011). Existing literature suggests that correctional officers may have negative perceptions of mentally ill offenders, and do not feel adequately trained or prepared to manage this population (Callahan, 2004; Kropp, Cox, Roesch \& Eaves, 1989; Petracek, 2012; Skeem, Manchak, \& Peterson, 2011). Evidence suggests that mental health training such as crisis intervention training and mental health first aid can educate and empower officers to implement mental health interventions, and improve perceptions of mentally ill offenders (Gibb, 2015; Parker, 2009; Petracek, 2012).

Discussion of the interactions of correctional officers and their understanding of offender mental illness must consider the context of correctional work. Undoubtedly, the nature of a career in corrections can be demanding. Correctional officers often experience high rates of stress and burnout that can lead to problems such as emotional fatigue, depersonalization, and diminished personal accomplishment (Finn, 2000; Lambert, Cluse-Tolar \& Hogan, 2007; 
Morgan, Van Haveren \& Pearson, 2002). It is important to understand the emotional and psychological well-being of officers. Emotional fortitude and effective interpersonal and occupational functioning can be assessed though measuring emotional intelligence (EI) (Pekrun, \& Frese, 1992; Salovey \& Mayer, 1990). Existing mental health training modalities in corrections and related fields often include components of emotional intelligence, and research suggests that EI can be increased through training (Al Ali, Garner, \& Magadley, 2012; Nelis et al., 2011; Schutte, Malouff, \& Thorsteinsson, 2013). EI can serve as a protective factor against job related burnout and perceived stress (Salovey, Bedell, Detweiler, \& Mayer, 1999; Slaski \& Cartwright, 2002). Additionally, higher EI leads to greater empathy, greater abilities to manage emotions, higher job satisfaction, lower turnover, more effective communication, and an increased willingness to take on leadership roles (Downey, Papageorgiou, \& Stough, 2006; George, 2000; Al Ali, Garner, \& Magadley, 2012; Salovey, Bedell, Detweiler, \& Mayer, 1999; Slaski and Cartwright, 2002).

Two out of ten offenders suffer from a mental illness, and correctional officers have more frequent interaction with mentally ill offenders than any other service or set of individuals within correctional systems. It is important to ensure that correctional officers feel adequately trained and have the resources and skills necessary to avoid depersonalization and burnout. The literature regarding the utility and efficacy of mental health training for correctional officers is scarce. A comprehensive understanding of correctional officer attitudes, and what contributes to, influences, and improves such attitudes is not currently available. A better understanding of the dynamic relationships among correctional officer experiences, opinions about mental illness, burnout, and emotional intelligence can assist in the formulation of training and education strategies and can facilitate more effective interdisciplinary cooperation. 


\section{Problem Statement}

Hundreds of thousands of incarcerated individuals in the United States suffer from mental illness. Therefore, it is imperative that correctional facilities adapt to manage the unique needs associated with offenders with serious mental illness (SMI). Correctional officers play an integral role in the daily functioning of jails and prisons and have the most frequent, direct contact with inmates (Adams \& Ferrandino, 2008; Fellner, 2006; Rhodes, 2004). Additionally, correctional officers can assist in providing effective interventions when mental health crises occur (Skeem, Manchak, \& Peterson, 2011).

Despite the importance of the role of officers in managing mental health related behaviors, there is limited literature that examines the attitudes that correctional officers maintain regarding a segment of the population with whom they work directly every day (Petracek, 2012; Skeem, Manchak, \& Peterson, 2011). Not only is our knowledge of correctional officer attitudes limited, there is an inadequate understanding of individual officer characteristics and experiences that may influence attitudes toward mental illness, such as emotional intelligence, levels of burnout, and involvement in mental health training.

Emotional intelligence refers to "the ability to monitor one's own and others' feelings and emotions, to discriminate among them and to use this information to guide one's thinking and actions" (Salovey \& Mayer,1990, p. 189). Literature suggests that emotional intelligence can be improved through training programs (Nelis et al., 2011), psychotherapy (Nelson, Low \& Ellis, 2007; Rieck \& Callahan, 2013), and education (Boyatzis, Stubbs, \& Taylor, 2002).

Training programs and educational programs that increase emotional intelligence have been highly sought-after in various service-work fields such as corrections (Kjerulf, 2014). Literature regarding the abilities of effective leaders in corrections parallels the abilities found in 
emotional intelligence (Newman, Guy \& Mastracci, 2009). For example, correctional officers must exhibit emotional intelligence when communicating with offenders and interpreting offender body language to prevent volatile situations (Stys \& Brown, 2004). Additionally, officers are responsible for the safety of their peers, themselves, the institution, and offenders, making emotionally intelligent decision-making imperative. (Manzella \& West, 2003). Despite the importance of high correctional officer emotional intelligence, literature suggests that correctional officer burnout rates are high, which may make monitoring one's emotions and engaging in emotionally intelligent thinking and actions difficult (Fin, 2000; Morgan, Van Haveren \& Pearson, 2002; Parker, 2009).

When combining the importance of emotional intelligence, with the issues of burnout, finding methods of supporting correctional officer emotional well-being, such as mental health training, is important. The data gathered through this study may assist in the development and implementation of training and policies within correctional settings. Additionally, it may facilitate further research in examining the problem more closely.

\section{Purpose of the Study}

The purpose of this study was to identify correctional officer opinions of offenders suffering from mental illness, and to understand the relationships among officer opinions, burnout, and emotional intelligence. Additionally, this study aimed to understand if mental health training for correctional officers can lead to higher emotional intelligence, more positive opinions about mental illness, and lower levels of burnout. This information could be useful for mental health professionals and administrative personnel to better understand the dynamic interplay between officers and offenders who suffer from SMI. This may consequently assist mental health professionals and facility administrators in working effectively as part of 
interdisciplinary teams and in determining interventions and training modalities for correctional officers.

\section{Significance of the Study}

Correctional officers endorse high levels of job stress, a lack of understanding of individuals affected by mental illness, as well as a desire for tools that can be employed when managing offender mental health problems and crises (Kropp, Cox, Roesch \& Eaves, 1989; Parker, 2009). The perspectives, concerns, and needs of correctional officers should be considered, as they play a crucial role in the operation of prisons.

The roles of correctional institutions are multidimensional. Within prisons, the maintenance of safety, prevention of escape, and minimization of suffering are paramount (Dvoskin \& Spiers, 2004). Exceptional safety operations, escape prevention, and suffering minimization can also result in higher staff morale and exceptional daily prison operations. This is a daunting task for correctional facilities' administrative leadership. Understanding the experiences of correctional officers and working to improve such experiences are in the best interest of correctional facilities at large.

Systemic issues, such as limited funding, often leave mental health departments in correctional environments understaffed. Additionally, psychologists spend nearly one-third of their time in correctional environments performing administrative responsibilities (Boothby \& Clements, 2000). This makes collaboration with prison staff an important component of management of the mental health symptoms of incarcerated individuals. Correctional officers have the most contact with offenders and may be the first to identify changes in offender behavior or mental status. Correctional officers may consequently be the first to alert mental 
health staff of such mental health changes when they occur (Appelbaum, et al., 2001; Dvoskin \& Spiers, 2004).

The current deficits in our understanding of correctional officer attitudes toward mentally ill offenders can lead to practical, interactional problems between officers and offenders that negatively impact the day to day operations of correctional facilities (Adams \& Ferrandino, 2008; Fellner, 2006; Rhodes, 2004). It is becoming increasingly important to gain a fundamental understanding of the contextual role and experiences of correctional officers, as well as their opinions about and ability to effectively interact with the growing number of mentally ill offenders housed in prisons across the United States. This quantitative study examined correctional officer opinions about mentally ill offenders, as well as the relationships between opinions, emotional intelligence, burnout, and mental health training.

\section{Research Questions and Hypotheses}

The following research questions and hypotheses were examined in the study:

1. What is the relationship between correctional officer opinions about mentally ill offenders and emotional intelligence (EI), as measured by the Opinions about Mental Illness scale (OMI) and the Schutte Self-Report Emotional Intelligence Test (SSEIT)?

a. Hypothesis 1: Higher, more negative attitude scores on the OMI will predict lower emotional intelligence scores (SSEIT).

2. Is there a significant relationship between correctional officer burnout, as measured by the CBI, and emotional intelligence, as measured by the SSEIT?

a. Hypothesis 2: Higher burnout scores on the CBI will predict lower emotional intelligence scores (SSEIT) 
3. Does burnout moderate the relationship between opinions about mental illness and emotional intelligence?

a. Hypothesis 3: Burnout (CBI) scores will moderate the relationship between opinion (OMI) scores and emotional intelligence (SSEIT) scores such that when the moderator is present, there will be a significantly stronger relationship between emotional intelligence and opinions.

4. Is there a significant relationship between exposure to mental health training and emotional intelligence as measured by the SSEIT?

a. Hypothesis 4: Greater exposure to mental health training will predict higher emotional intelligence (SSEIT).

5. When controlling for mental health training, is there a significant relationship between opinions about mental illness, as measured by the OMI, and emotional intelligence, as measured by the SSEIT?

a. Hypothesis 5: When controlling for mental health training, higher, more negative opinions about mental illness (OMI) scores will predict lower emotional intelligence (SSEIT) scores.

6. Does exposure to mental health training moderate the relationship between opinions about mental illness and emotional intelligence in correctional officers?

a. Hypothesis 6: Exposure to mental health training will moderate the relationship between opinion scores (OMI) and emotional intelligence (SSEIT), such that when the moderator is present, there will be a significantly stronger relationship between emotional intelligence and opinions about mental illness. 


\section{Definitions of Correctional Concepts}

1. Offender/Inmate - an individual who is legally held in prison as punishment for being convicted of a crime.

2. Correctional Officer (CO) - an individual employed by prisons who is responsible for the supervision, safety, and security of offenders and the facilities that house offenders.

3. Corrections - a network of agencies that are responsible for the administration of prisons and community-based programs such as probation and parole.

4. State Prison - a prison operated and maintained by a state in the United States of America that is used to house and rehabilitate offenders.

5. Federal Prison - a prison operated and maintained by the federal government of the United States of America that is used to house and rehabilitate offenders.

6. Recidivism - a relapse in criminal behavior such as a re-arrest, re-conviction, or re-entry into prison after having received legal sanctions and served a sentence for a previous crime (National Institute of Justice, 2014).

\section{Operational Definitions}

1. Serious Mental Illness (SMI) - a diagnosable mental, behavioral, or emotional disorder that meets diagnostic criteria specified within DSM-5 and that significantly interferes with functioning (National Institute of Health, 2017).

2. Opinions - the correctional officer's perspective about and attitude toward persons with mental illness, specifically regarding the etiology, prognosis and treatment of mental illness, as reported on the Opinions about Mental Illness (OMI) scale (Cohen, \& Struening, 1962), ranging from 1 (strongly disagree) to 6 (strongly agree). 
3. Emotional Intelligence (EI) - an individual's ability to be conscious of, control, and express one's emotions, and the ability to effectively manage interpersonal relationships with wisdom and empathy as reported on the Schutte Self-Report Emotional Intelligence Test (SSEIT) (Schutte, et al., 1998), ranging from 1 (strongly disagree) to 5 (strongly agree).

4. Mental Health Professional - a health care provider who offers services aimed at improving an individual's mental health or treating mental disorders (National Alliance of Mental Illness, 2017).

5. Interdisciplinary team - a group of professionals from differing fields who work collaboratively toward common goals for mentally ill offenders (Bronstein, 2003).

6. Mental Health Training - programming offered by a facility that enables participants to identify, understand, and respond to signs of mental health problems or crises.

\section{Summary of the Study}

This study attempted to add to the existing literature and expand the current knowledge about correctional officer opinions of mentally ill offenders. The study further attempted to examine the relationships between officer opinions about mentally ill offenders, emotional intelligence, burnout, and mental health training. Further research on these factors may assist in the development and implementation of training opportunities and policies within correctional settings that enhance correctional officer EI and decrease officer burnout. 


\section{CHAPTER 2: REVIEW OF THE LITERATURE}

\section{Historical Context}

Currently, approximately 20 percent of incarcerated individuals in the United States suffer from a serious mental illness (SMI) (Aufderheide, 2014; Baillargeon et al., 2009; Prins, 2014). In jails and state prisons alone, this equates to over 356,000 affected individuals (Center, 2014). Glaze and Herberman (2012), commissioned by the Bureau of Justice Statistics, found that within state prisons, up to four percent of offenders have a psychotic disorder, up to 18 percent have major depression, and up to four percent have bipolar disorder. In the United States, SMI individuals are three times more likely to be incarcerated than placed in a mental health facility. Further research suggests that, of individuals with a SMI, 40 percent will encounter community corrections, jail or prison at some point in their lives (Aufderheide, 2014). Correctional systems have become America's largest mental health facilities, a purpose for which they were not intended (Baillargeon et al., 2009).

Prior to the 1960's, individuals with SMI living in the community were primarily treated in psychiatric hospitals and institutions (Novella, 2010). Such facilities were controversial due to issues such as understaffing, over-crowding, human rights violations, and generally poor conditions (Burnham, 2006). In the 1940's, various investigative publications, including Deutsch's 1948 The Shame of the States, were released, uncovering deplorable conditions in mental institutions (Burnham, 2006; Deutsch, 1948; Primeau, Bowers, Harrison, \& Xuxu, 2013). This served as a catalyst for conversations regarding treatment of the mentally ill. In the United States, the civil rights movement of the 1960's created a social climate that was conducive to systemic change in the way that society approached the treatment of those suffering from mental illness (Kliewer, Melissa, \& Trippany, 2009). As a result, a push toward deinstitutionalizing 
individuals with mental illness began. The 1960's brought a shift in focus from mental institution warehousing to seeking community-based, outpatient services (Novella, 2010). Concurrently with this social change came the introduction of antipsychotic medication. By the mid-1950's, effective antipsychotic drugs were becoming readily available to the public (Anfang \& Applebaum, 2006; Novella, 2010). The culmination of these changes brought about the Community Mental Health Centers Act of 1963 (Feldman, 2003).

The Community Mental Health Centers Act of 1963 (Feldman, 2003) significantly altered the way that mental health services were delivered. In addition, more effective psychotropic medications were developed, and there was increased innovation in approaches to psychotherapy. Additionally, community mental health centers were funded and established across the United States, and institutionalized individuals were re-integrated into their communities (National Council for Behavioral Health, 2015).

\section{Mental Illness and Corrections}

For people suffering from mental illness, crises are not always solved through the efforts of community mental health centers or inpatient treatment facilities. Individuals with a SMI are more likely to encounter law enforcement than to seek medical treatment (National Alliance on Mental Illness, 2015). Because of these interactions, nearly 400,000 individuals with SMI find themselves in jails and state prisons each year (Torrey, et al., 2014). Jails and prisons now house more mentally ill individuals than ever. In fact, jails and prisons hold 10 times more individuals suffering from SMI than state hospitals (Kaeble, Glade, Tsoutis, \& Minton, 2016).

Various systemic problems arise from the incarceration of individuals with mental illness. Once placed in jail, individuals may not have access to needed mental health services and are more likely to decompensate (National Alliance on Mental Illness, 2015). Further, individuals 
with SMI often stay in jail longer than their non-SMI counterparts (Fuller et al., 2016). Once released, a criminal record may prevent them from accessing health care, employment, or housing. This may lead to homelessness or elevated levels of recidivism (Geller \& Curtis, 2011; Visher, 2007). Thousands of offenders with SMI and limited access to psychiatric services may experience the revolving door of re-incarceration (Baillargeon et al., 2009).

Once individuals with SMI have entered into a correctional setting, they begin to interact with various systems present within correctional settings. One such system they encounter is safety and security. Ensuring the safety and security of a correctional facility is primarily tasked to correctional officers (COs) (Bureau of Labor Statistics, 2017). COs have a range of responsibilities, including enforcing rules, maintaining order, supervising activities, searching for contraband, inspecting facilities, and reporting inmate conduct violations

(CorrectionalOfficer.org, 2016). Of staff within correctional settings, COs have the most direct contact with offenders, including offenders with SMI (Galanek, 2015).

\section{Mental Health Treatment in Corrections}

In the 1970's, the United States Supreme court ruled that "deliberate indifference" regarding medical necessities of incarcerated individuals was a violation of the eighth amendment to the United States constitution (Rold, 2008). The eighth amendment bans "cruel" and "unusual" punishments of criminal defendants (Stinneford, 2017). As a direct result, jails and prisons are now required to establish medical practices that promote "decency and dignity" (Shenson, Dubler, \& Michaels, 1990). To adequately treat offenders with SMI in correctional settings, treatment units have been created at some facilities (Anasseril, 2007). A 2001 Survey found that in 1,394 of the United States' 1,558 adult state prisons, 51 percent provide 24-hour acute mental health care (Beck \& Maruschak, 2001). These numbers are low when compared to 
other services offered. The same survey found that 70 percent of these prisons screen offenders for mental health concerns at intake, 65 percent provide psychological assessments, 71 percent provide therapy, and 73 percent provide psychotropic medications (Beck \& Maruschak, 2001). The American Psychological Association (APA) and the National Commission on Correctional Health Care (NCCHC) have established recommended guidelines for assisting correctional facilities in creating mental health services (American Psychological Association, 2017; National Commission on Correctional Health Care, 2015). Per their recommendations, facilities should offer mental health screenings, crisis intervention, substance abuse treatment, psychiatric medication management, and specialized treatment units (Hills, Sigfried, \& Ickowitz, 2004). Within state prisons, many facilities have shifted funding to create acute psychiatric units (Lamb \& Weinberger, 2005). There are both limitations and benefits of acute psychiatric units within a correctional setting.

\section{Disadvantages of Acute Units}

Within corrections, concerns may arise regarding how to best implement acute psychiatric care units. Areas of concerns include deciding criteria for admission, level of care, types of therapies used, and access to medical records (Anasseril, 2007; Cloyes, 2007). Additionally, the handling of conduct violations by offenders with SMI can be challenging. For example, it may be difficult to determine if an SMI offender was competent at the time of the conduct violation (Anasseril, 2007).

Acute psychiatric units can create expenses and security concerns related to transferring offenders back and forth between treatment units and the general population (Anasseril, 2007). Interdepartmental miscommunications can also occur regarding where an offender should be housed, and the level of treatment they should be receiving. This is an inherent challenge of the 
correctional environment. For example, handling and moving offenders is much different than handling traditional non-offender psychiatric patients (Anasseril, 2007).

\section{Advantages of Acute Units}

Despite the disadvantages of acute units in prisons, there are also identifiable advantages. Acute units can create a therapeutic milieu and provide treatment while respecting the facility's mission of ensuring the safety and security of offenders, staff, and the institution (Anasseril, 2007). Additionally, they can promote the safe and appropriate implementation of psychiatric treatments. For example, acute units can safely address medication non-compliance. In the Supreme Court case of Washington v. Harper, the court ruled that dangerous offenders cannot refuse psychiatric treatment via antipsychotic medication (Applebaum, 1990). Involuntary medications can be administered to non-compliant, gravely ill offenders per this ruling, and this can be done effectively in acute units (Anasseril, 2007).

\section{Mental Health Professional and Correctional Officer Differences}

Mental health providers generally have a culture that values individualized treatment, empathic concern, informed consent, and negotiation (Behnke, 2001). Mental health professionals (MHPs) may often feel at odds with safety and security in correctional settings due to divergent perspectives regarding job title and professional culture. The primary objective of the $\mathrm{CO}$ is to maintain safety and security, while the primary objective of the MHP is to promote health and psychiatric treatment (Appelbaum, Hickey, \& Packer, 2001; Mauro, 2009; Sun, 2013).

Although COs and MHPs work collaboratively, these contrasting ideologies can cause conflict. Appelbaum et al. (2001) suggested that COs often perceive MHPs as overly trusting, excessively lax, and coddling toward offenders. Further, they stated that officers view mental 
illness as "character flaws" and perceive that mental health treatment shelters offenders from receiving consequences for negative behaviors (Appelbaum, et al., 2001). It is important for these interacting systems to mediate this perception of difference to work effectively as a team in the best interest of institutions and the offenders they house (Start, 1998; Steadman, 1992; Steadman, McCarthy, \& Morrissey, 1989).

\section{Correctional Officer Perceptions and Attitudes}

Kropp, Cox, Roesch, and Eaves (1989) sought to better understand CO perceptions, given the frequency of interaction that officers have with offenders with SMI. Utilizing a perception scale, researchers found that, as compared to non-psychiatrically disordered offenders, offenders with mental illness were perceived as more dangerous and violent, but less manipulative and less able to control their environment. Namely, the study found that COs endorsed mentally ill offenders as unpredictable, irrational, and mysterious (Kropp, et al., 1989). Despite mental health training and education related to psychopathology, COs still maintained negative perceptions of offenders with mental illness, and an assumption of the uniform dangerousness of offenders with mental illness (Kropp, et al., 1989).

Published, peer-reviewed research regarding CO perceptions of offenders with mental illness has expanded only minimally since Kropp et al.'s research in 1989. In 2004, Callahan utilized a vignette type scale to assess CO perceptions of offenders with mental illness and obtained a large sample of over 1800 responses. All respondents had had some exposure to mental health training, per the mandates of their respective facilities (Callahan, 2004). Results of the study suggested that officers were able to correctly differentiate between schizophreniarelated symptoms and symptoms of depression. Additionally, officers identified violence as being associated with mental illness in the vignettes and they perceived that offenders who 
experience schizophrenia should not have autonomy in treatment decisions (Callahan, 2004).

While the study reveals that COs are effective in correctly identifying schizophrenia and depression, it is also noted that COs strongly favor coercion of treatment. This may be due to the perception that severely mentally disordered offenders are violent (Callahan, 2004).

Since Callahan's (2004) study, various dissertations and academic works have attempted to better understand CO attitudes toward offenders with mental illness. Keeler (2017) compared $\mathrm{CO}$ and licensed psychologist perceptions of offenders with mental illness. The results suggested that $\operatorname{COs}(N=141)$ have more negative perceptions of general mental illness and inmates that have mental illness than licensed psychologists. Further, the results indicate that the more negatively COs viewed mental illness, the more likely they were to perceive individuals with mental illness as dangerous, a burden to society, lacking self-discipline, and needing to be hospitalized (Keeler, 2017). Additional results of the study suggest that more negative perceptions of offenders with mental illness were associated with viewing mental health services as a waste of money, and the view that offenders with mental illness are manipulative and stressful (Keeler, 2017).

A dissertation conducted by Harmon-Nary (2013) surveyed 108 COs at a maximumsecurity state prison in Indiana. The study examined perceptions of mental illness but also allowed COs to indicate their perceptions across different psychiatric diagnoses. Results suggested that COs felt that offenders with depression were more likely to be rehabilitated than offenders with psychosis, mania, and personality disorders. Offenders with a psychotic diagnosis were viewed as unsafe and too dangerous to be around other inmates as compared to other diagnoses. Offenders with antisocial personality disorder were viewed as more predictable and less stressful to work with than offenders with psychotic disorders. Overall, offenders with 
psychotic disorders were seen as the least predictable, as compared to individuals with other diagnoses. Participating COs indicated that they could benefit from more mental health-related training and agreed that they could influence treatment by offering offenders positive support (Harmon-Nary, 2013).

Powers-Margo (2015) examined California jail COs'stress, job satisfaction, and perceptions of mentally ill inmates in a qualitative dissertation. There were 11 participants in the study, and all worked within a psychiatric unit in a county jail. Various themes emerged from the study, including that officers viewed offenders with mental illness as child-like, and requiring more patience than mainline offenders. Participants also reported that training, camaraderie and support were among factors that decreased their stress and increased job satisfaction (PowersMargo, 2015).

Some of the studies of CO perceptions of offenders with mental illness have found that mental health training for COs has many potential benefits, including the mitigation of negative perceptions (Applebaum, Hickey, \& Packer, 2001; Davidson, 2004; Powers-Margo, 2015). Having an understanding of and the necessary skills to assist in mental health crises can promote the safety of officers and offenders. Additionally, facility security and the humane treatment of offenders are promoted with proper training (Parker, 2009). Understanding the utility and implementation of mental health training can help us to better serve the needs of both officers and the offenders that they serve (Parker, 2009).

\section{Correctional Officer Personality Traits}

In the field of corrections, it is valuable to understand the personality variables associated with officers (Holland, Heim, \& Holt, 1976). Identifying character traits enables us to better understand the world view and experiences of those working in the field. This may have an 
impact on the interactions that COs have with the SMI offenders that they assist. Holland and colleagues (1976) attempted to identify personality patterns among applicants for CO positions. As part of the pre-employment application process, a large sample of California applicants was given the Minnesota Multiphasic Personality Inventory (MMPI). Results were based on average applicant scores and indicated that applicants completed the assessment in a defensive manner (Holland, Heim \& Holt, 1976). Further, the average profile revealed elevations on scales 9 (Hypomania) and 4 (Psychopathic Deviate), followed by elevations on scales 3 (Hysteria) and 5 (Masculinity). Based on the results of the study, applicants fitting these personality profiles were likely to be outgoing and talkative as well as manipulative within their social environments. Results suggest that COs with this profile would be goal driven, yet have difficulty understanding the needs and concerns of others (Holland et al., 1976).

Research conducted by Borman, Penner, Allen, and Motowidlo (2001) suggests that, as some COs begin to adjust to their roles, they are less likely to exhibit job enthusiasm, rule following, and cooperation with others. Personality and temperamental factors can also influence stress and coping. Strelau (1998) defined temperament as personality traits and related behaviors that are present in early childhood. Temperament has been found to play a regulative role when individuals are under stress and can play a moderating role in coping and stress outcomes. Cieslak, Korczynska, Strelau, and Kaczmarek (2008) found that correctional officer temperament can moderate the relationship between work stress and burnout. The personality trait of endurance, defined as level of efficiency of behavior under stress, was specifically found to play an important role in stress (Cieslak et al., 2008). Correctional officers with lower endurance reported higher levels of work stress, less social support from peers, higher levels of emotional exhaustion, and lower levels of sense of accomplishment (Cieslak et al., 2008). 


\section{Correctional Officer Burnout}

The job-related demands of COs have increased along with the growth of the U.S. prison population, and research has been dedicated to how COs respond to changes in their responsibilities (Misis et al., 2013). An area of interest has been relationships between COs, and the physiological and psychological aspects of stress (Brough \& Williams, 2007; Carlson, Anson, \& Thomas, 2003; Schaufeli \& Peeters, 2000). Research suggests correctional work is demanding, stressful and poses risks to individual health and safety (Cullen, Link, Wolfe, \& Frank, 1985; Liebling, Price, \& Shefer, 2011; Spinaris et al., 2012).

Identifying the unique job characteristics that have the most impact on COs is foundational in understanding the effect of the prison environment on officers. Lambert, CluseTolar, and Hogan (2007) attempted to assess job characteristics and their impact on CO stress. Researchers utilized self-report measures to survey $\mathrm{CO}$ job stress variables as well as job characteristic variables. Results suggested that, as job characteristics such as job-related danger, role stress, and job involvement increase, COs report higher job stress. Further, results suggest that variables within job characteristics have a more significant impact on COs than personal characteristics, suggesting that the job climate may play a role in shaping CO stress (Lambert, Cluse-Tolar \& Hogan, 2007). Another notable result was that the provision of feedback on CO job performance can have a significant effect on $\mathrm{CO}$ stress, suggesting that effective, meaningful performance feedback reduces stress (Lambert et al., 2007). Results suggest a need for better intervention within prisons at a systemic level to reduce job related stress.

Although CO burnout is high, there are methods by which to ease the impact of jobrelated stressors. Finn (2000) argued that managing officer stress is imperative to the functioning of correctional facilities. Stress management programs have various benefits, including 
improvement in officer performance, increased safety of the institution, and more positive inmate interactions. Finn (2000) also offered key elements of programs that successfully manage CO stress, including dedicated staff, administration and stakeholder support, confidentiality, and program evaluation. Stress reduction may lead to healthier and better equipped officers who are committed to their roles and interact more effectively with the offenders they encounter each day (Finn, 2000).

Additional inquiry into CO stress has yielded information regarding $\mathrm{CO}$ burnout. Burnout can lead to professional difficulty and stems from the exhaustion of both physical and mental resources. Lindquist and Whitehead (1986), as cited in Morgan, Van Haveren, and Pearson (2002, p. 146), found that “... 68\% of correctional officers considered their job to be at least moderately stressful, and approximately 33\% reported experiencing symptoms of burnout." Participants in Morgan et al.'s (2002) research completed a burnout questionnaire. Results suggest various demographic and work-related variables that may further impact CO stress. Younger COs endorsed higher levels of burnout, as did those with higher potential for exposure to violence. One mitigating factor of burnout identified in the literature was peer and social support. Quality of contact with offenders may also be a mitigating factor for officers, as the potential of violent encounters leads to higher levels of burnout (Morgan, Van Haveren \& Pearson, 2002). This factor may support the need for officer training in offender interactions.

According to Parker (2009), COs play an essential role in maintaining the safety and security of a correctional facility; however, various challenges are associated with this occupation. Research suggests that COs may experience various stressors associated with their careers. Long work hours, low pay, and risk of violence can all contribute to stress among officers (Parker, 2009). These added stressors make the management of SMI and conduct issues 
in prisons a balancing act. Conflicts within the treatment-custody paradigm often require that COs use a great deal of judgment. Exposure to mental health training may mitigate the impact of CO stressors and SMI offender conduct (Adams \& Ferrandino, 2008).

\section{Mental Health Training in Corrections}

Mental health training has various benefits for correctional officers, including effective collaboration and an increased knowledge of ethical issues, legal issues, and national standards of care (American Correctional Association, 2017; Applebaum, Hickey, \& Packer, 2001). Unfortunately, there is no uniform mental health training mandated for correctional officers across state correctional facilities. However, most states have begun to include components such as mental health education into classroom-based training (Correctional Officer Education, 2018). The limitation of this classroom-based training is that the only exposure to mental health issues may be discussed in a cursory manner in pre-service training (Spearlt, 2009). Some facilities do offer specialized mental health training and certification to officers working in mental health units, or other interested COs (Adams-Flores, et al., 2017; Parker, 2009). As COs have extensive contact with offenders, in and out of treatment units, knowledge of psychiatric disorders as well as effective interventions within this population may be useful (Applebaum, Hickey, \& Packer, 2001). More research is needed to better understand the impact of mental health training on CO dispositions and attitudes toward offenders with mental illness.

As recently as 2012, researchers have attempted to better understand the efficacy of mental health training for CO’s. Petracek (2012) found that COs often report not feeling adequately trained to manage the challenges that may be associated with offenders with mental illness. To better understand CO perceptions and the efficacy of mental health training, Petracek's (2012) research surveyed officers and found that COs, in a county jail setting in 
Minnesota, who received crisis intervention training (CIT), maintained similar perceptions of offenders with mental illness as their non-trained counterparts. However, CIT-trained officers felt better equipped to work with offenders with mental illness. Perceived ability to work with offenders with mental illness scores also improved with length of time working at the facility. More than 60 percent of surveyed officers indicated that the facility did not offer enough training regarding mental health, while 80 percent indicated a belief that mental health training contributes to the safety and security of the facility (Petracek, 2012). The results of this study suggest that, while officers may maintain negative perceptions of offenders with mental illness, they perceive mental health training to be beneficial.

Currently, there are various forms of mental health training that have been implemented in jails and state prisons across the United States. In association with the National Alliance on Mental Illness (NAMI), Indiana correctional facilities implemented mental health training for COs working on special housing units for offenders with SMI (Parker, 2009). The program included two-hour sessions, once per week, for five weeks. First session topics included an introduction to diagnostic categories for mental illnesses, along with case examples. The second session addressed the biology of mental illness, including neurological diagrams and examples. The third session discussed treatment of mental illness, including therapy and medication management. The fourth session included effective means of interacting with individuals with SMI. The final session was an integration of the prior sessions, and included role-plays (Parker, 2009). The training curriculum was field tested by correctional officials at a training conference hosted by NAMI. Typically, when the work-related pressures of COs combine with the unique challenges associated with interactions with offenders with SMI, the potential use of force increases. However, Parker (2009) suggested that mental health training can significantly 
decrease the use of force toward SMI offenders. This research also found that after mental health training was completed, the number of incidents involving use of force and battery with bodily waste decreased significantly in the year that followed, as compared to the months preceding the training. COs may be able to play an integral role in the treatment and maintenance of offenders with SMI if they receive the proper training, especially in special and segregated housing units.

Another approach that has been taken in the effort to train COs in working with offenders who have mental disorders is "Mental Health First Aid (MHFA)" (Gibb, 2015). In Pennsylvania, the Department of Corrections implemented the use of MHFA and have trained over 15,000 officers since its implementation (Gibb, 2015). MHFA has various benefits, including its availability to the public and its use of a skills-based, in-person curriculum. This eight-hour training encompasses five steps, including recognizing signs and symptoms of mental illness, identifying tools and resources, referring to supportive services, and de-escalation (Gibb, 2015). Although no outcome data are available at this time, correctional staff provided feedback for the training program. Feedback suggests that COs felt more educated and empowered to implement the intervention strategies that they learned (Gibb, 2015). In the future, MHFA will be included in pre-employment training as a competency requirement in the Pennsylvania Department of Corrections.

Not only did Pennsylvania incorporate MHFA training for COs, they engaged in an overhaul of the policies and procedures regarding the treatment of offenders with mental illness (Pennsylvania Department of Corrections, 2016). The Department of Corrections implemented Crisis Intervention Team training, Mental Health Performance Contracting for accountability, Mental Health Advocates, Suicide Prevention committees, and established an ongoing partnership with NAMI. These actions, spurred from a federal investigation into the treatment of 
offenders with mental illness, were so effective that the investigation was closed (Penn. DOC, 2016). Pennsylvania's new-found commitment to mental health care is expected to significantly improve the quality of care given to inmates with mental illness and intellectual disabilities.

The Department of Corrections in Washington State also attempted to address the increasing need for COs to receive mental health training (Gillepie, 2015). The Crisis Intervention Training, implemented in Washington jails, was one week-long, and consisted of 40 hours of crisis training. The program, created by a facility sergeant and former president of the local chapter of NAMI, was comprised of lectures, hands-on training, and role-play activities (Gillepie, 2015). Community partners engaged in the process and provided actors for the roleplay activities. Actors exhibited symptoms of various disorders, including schizophrenia and posttraumatic stress disorder. The program was perceived as effective by correctional staff, and the Department of Corrections intends to offer the course twice a year for officers (Gillepie, 2015).

With training, officers are better able to engage in therapeutic communication, consultation, and medication and side-effect observations (Dvoskin \& Spiers, 2004). Because of this, mental health training may be beneficial for not only offender interactions, but also for consulting and working in an interdisciplinary setting with mental health professionals (Compton, et al., 2006; Dvoskin \& Spiers, 2004; Steadman et al., 2000). It may be useful to consider effective training modalities that have been employed in other law enforcement careers to better understand the available interventions that have been empirically tested.

\section{Mental Health Training in Related Fields}

With the increase in individuals with mental illness encountering law enforcement, police officers have also landed on the front lines of mental health crises. Prior to their interactions with 
COs, people with SMI have likely encountered members of the police force (Lamb, Weinberger, \& DeCuir, 2005). With the growing demand to meet the needs of SMI community members, many police departments across the country have employed training to effectively interact with affected persons (Lamb, et al., 2005). In addition to interacting with individuals with mental illness, police officers may make decisions regarding entry into correctional systems, or mental health systems (Lamb et al., 2005). For example, after a police referral is made, officers may be required to decide if incarceration or psychiatric treatment is the most appropriate option for the person in crisis. Further, police have a duty to protect the safety of their communities, as well as to protect persons with disabilities. The duty to protect and maintain order in communities in general parallels that of correctional communities. Therefore, it may be valuable to understand the mental health training required of front-line officers to better inform the mental health training practices provided to COs.

Law enforcement respond to community mental health crises in many ways. One program that is widely referenced in existing literature is Crisis Response Training (CRT), a model that has also been implemented in some correctional settings (Compton et al., 2006; Steadman et al., 2000; Teller, Munetz, Gil \& Ritter, 2006). The primary objective of CRT is to educate and provide skill sets to officers who encounter individuals with mental illness. Compton and colleagues (2006) assessed the impact of CRT on police officer attitudes toward individuals with schizophrenia. The CRT certification consists of a 40-hour course, and officers are given pre- and post-tests to assess changes in attitudes following the training. Researchers administered the program to 159 officers in the Atlanta Metropolitan Area. The results of the study suggest that CRT does have an impact on officer perceptions. Officers reported improved attitudes regarding the aggressiveness of individuals with schizophrenia $(p=0.1)$, increased support for 
mental health treatment programs $(p<.01)$, increased support for substance abuse treatment programs $(p<.01)$, increased knowledge about schizophrenia $(p<.001)$, and lower perceived social distance from individuals with schizophrenia $(p<.001)$ (Compton et al., 2006). It appears that CRT can have a profound impact on the way that law enforcement officers view SMI, which may influence the way in which interactions occur.

Teller, Munetz, Gil, and Ritter (2006) assessed the utility of CRT through the analysis of police dispatch calls for two years pre-and four years post-CRT certification. The study was conducted in Akron, Ohio, and the training was implemented through NAMI. Police Department calls between May 1998 and April 2004 were included in the study. Of the 1,527,281 calls, 10,004 were associated with mental illness. Throughout the duration of the study, the rate of mental health-related calls increased. Despite the increase in demand for response to mental health crises, researchers found that, as compared to responding officers not CRT-certified, CRT responding officers were more likely to transport individuals to treatment facilities than jail (Teller et al., 2006). CRT officers were also more likely to identify a call as a mental health crisis and to de-escalate a situation. The intention for police forces to implement CRT may be to promote the safety of officers, but the outcomes are two-fold. Not only is officer safety enhanced, the well-being of individuals with mental illness is improved (Teller, et al., 2006). Individuals with mental illness are less likely to have punitive outcomes and are more likely to receive treatment. This is an outcome that often better serves affected individuals.

Another unique approach that law enforcement teams have utilized is multi-disciplinary response. A multi-disciplinary approach, or "co-response" occurs when community mental health providers and police offers work collaboratively to respond to a person who is emotionally disturbed (Shapiro, et al., 2015). The primary objectives of co-response teams are de-escalation, 
prevention of injury to those responding, linking those in crisis into mental health services, and the removal of pressure from law enforcement. Shapiro and colleagues (2015) conducted a metaanalysis, reviewing programs that co-respond to mental health crises with law enforcement and mental health professionals. The study included 11 peer-reviewed articles, seven reports, and three dissertations. All resources were reviewed, and data was collected that assisted researchers in identifying important aspects of co-responding police and mental health programs. The primary elements of this type of program are officer mental health training, police and mental health partnerships, and organizational buy-in (Shapiro et al. 2015). Further, the elements that make these programs effective are awareness of mental health issues among officers, and awareness of co-response teams as a resource within police departments and the community. The primary outcome for this approach was fewer arrests of persons with mental illness. Through a review of the literature, this study also found several key themes associated with co-response teams, including the aversion of crisis escalation, positive effects of mental health treatment connections, reduction of hospital admissions, more positive officer perceptions of mental illness, and cost effectiveness (Shapiro et al., 2015). A multi-disciplinary approach or "coresponse" seems to mitigate the burden of crisis response on police officers, as well as creating positive outcomes for those in crisis while creating a sense of community among mental health professionals and law enforcement.

As law enforcement has utilized various methods to tackle the concern of the rising need for mental health intervention, Steadman, Deane, Borum, and Morrissey (2000) attempted to compare various response models for efficacy. Researchers assessed three different police departments to examine response programs. Birmingham Alabama and Memphis Tennessee 
utilized a police-based specialized response team, while Knoxville Tennessee utilized a mental health-based response.

In the Birmingham model, law enforcement affiliates wore plain clothes, and had a degree or specialized training in social work and related fields. Additionally, they participated in six weeks of crisis training. However, they were not sworn in as police offices, did not make arrests, and did not carry weapons (Steadman et al., 2000). These affiliates solely responded to mental health-related calls. Similarly, the Memphis model is police-based, but this response approach used a 40-hour CRT developed through partnerships with nearby universities and mental health agencies. In addition to regular patrol duties, Memphis officers certified in CRT responded to mental health crises (Steadman et al., 2000). In a contrasting manner, the Knoxville model utilized a mobile, nine-person mental health service team, serving five counties (Steadman et al., 2000). This model employed services around the clock, typically through a triage drop-off center with a "no refusal" policy. This provided services to all individuals who appeared to be in need of emergent mental health services.

Information was gathered from each department through dispatch calls and specialized incident reports (Steadman et al., 2000). Each model posed different barriers to serving mentally ill community members in crisis. The Birmingham model also had limited officer resources due to the small number of community officers trained to respond to crises (Steadman et al., 2000). The Knoxville model, because of the small number of team members, had a slow response time, preventing officers from utilizing the service. Because a majority of the officers in the Memphis CIT model were trained to respond to mental health crisis, the officers were readily available to be dispatched to mental health crises. Although the response rate of the Memphis CIT model was more efficient, crises were resolved on scene or referred for services less often than in the 
remaining two models (Steadman et al., 2000). There appear to be benefits and barriers in each of the three models. Small, specialized mental health teams appear to be more successful at resolving mental health crises; however, such teams may become overwhelmed by the demand for responses within their communities (Steadman et al., 2000).

The research regarding CO perceptions of offenders with mental illness, as well as the efficacy of $\mathrm{CO}$ mental health training, although limited, seems to have drawn similar conclusions. The intersection of criminality and mental illness appear to have a profound impact on the perceptions that COs maintain regarding SMI offenders, and limit CO's perceived ability to manage this population. Despite these challenges, the literature suggests that more correctional facilities are beginning to see the value of implementing mental health training (Compton et al., 2006; Shapiro et al., 2015; Steadman et al., 2000; Teller et al., 2006). Various types of programs emphasizing mental health and crisis have been utilized and are reviewed positively by correctional staff. One promising line of inquiry into improved interpersonal functioning is emotional intelligence (EI). The literature surrounding EI suggests that emotions play an integral role in our ability to understand ourselves and others. Because of the unique nature of correctional work, it is important to assess the value of EI on stress and interpersonal effectiveness within this profession.

\section{Emotional Intelligence}

History and context. The concept of EI was first introduced in 1990 by Salovey and Mayer, who purported that EI is a form of social intelligence. According to Salovey and Mayer (1990, p. 189), EI is defined as “the ability to monitor one's own and others' feelings and emotions, to discriminate among them and to use this information to guide one's thinking and actions." The pair posited that life tasks and cognition are infused with emotional information 
that must be processed. How people process emotional information differs from person to person. Emotional data is not limited to that of the self, but also includes information from our social environments. From this perspective, EI is used to perceive and utilize emotion for both problem-solving and monitoring behavior (Salovey \& Mayer, 1990).

The theory was expanded by Goleman (1996) and gained further popularity because of this work. In his best-selling book Emotional Intelligence: Why It Can Matter More Than IQ, Goleman defined EI as a skill set which includes one's ability to control their impulses, and one's ability to be self-motivated, empathic, and competent in social relationships. The implications of this publication were far reaching, as EI became a foundational theory in socioemotional learning which is applicable across various populations and settings. EI has an array of practical benefits, as research has linked high EI to prosocial behavior, self-confidence, motivation to achieve, and conscientiousness (Brackett, Rivers, \& Salovey, 2011). This took EI outside of the realm of basic problem solving into a broader, more pervasive context.

EI differs from traditional forms of intelligence. Our knowledge of the intelligence quotient (IQ) is vast and well researched. Broadly, IQ can be defined as "The aggregate or global capacity of the individual to act purposefully, to think rationally, and to deal effectively with his [sic] environment" (Wechsler, 1958, p. 3). Intelligence, from this perspective, pertains to an individual's cognitive abilities, whereas EI pertains to an individual's emotional abilities. Theories of EI were developed, in part, because traditional measures of intelligence did not account for the perception, processing, and management of emotional information (Mayer \& Salovey, 1997). EI does not fundamentally oppose cognitive definitions of intelligence, but rather acknowledges the unique intersection of the two concepts (Brackett, Rivers, \& Salovey, 2011). 
Conceptual framework. Conceptually, EI can be understood as mental processes that deconstruct emotional information. According to Salovey and Mayer (1990), such mental processes occur in three ways, "appraising and expressing emotions in the self and others, regulating emotion in the self and others, and using emotions in adaptive ways" (p. 190-191). The appraisal and expression of emotions begins when individuals first perceive affective information. EI, in turn, allows emotional information to be correctly evaluated and conveyed (Salovey \& Mayer, 1990). The means by which emotion is transmitted is often verbal expression. The appraisal of emotion in others is more closely linked to perceiving nonverbal emotions in others and experiencing and conveying empathy (Salovey \& Mayer, 1990). This allows individuals to gauge the emotions of others and to behave in socially formative ways. In appraisal, emotion is evaluated in an internal, subjective manner. This conceptual process requires emotional intellect, insofar as it necessitates internal processing, and accurate perception and expression of emotion.

The second mental process outlined by Salovey and Mayer (1990) is the regulation of emotions as it relates to the self and others. Regulating one's own emotions and mood states requires monitoring, gauging, and adapting. One's ability to navigate emotion influences the way in which they are perceived by others, making this process central in mood regulation (Salovey \& Mayer, 1990). An individual with high levels of EI is able to not only appraise and regulate emotion, but also to utilize it effectively. The final mental process in EI is utilization of emotions, which allows for problem solving. Using emotions can occur in several ways, including flexible planning, creative thinking, mood re-directed attention, and motivation (Salovey \& Mayer, 1990). This conceptual framework, comprised of appraisal regulation, and use, has operationalized EI for further research. 
Primary models. From the conceptual framework of EI, differing models of measuring the construct have been derived. There are two primary models of EI that are widely used in existing literature: the ability model and mixed models (Brackett, Rivers, \& Salovey, 2011). From the perspective of the ability model, EI is no different than traditional models of intelligence (Mayer, Roberts, \& Barsade, 2008). Per the criteria of intelligence, EI is a mental ability and can be measured via assessment of performance. These assessments contain criteria for correct and incorrect response. Ability models differ from mixed models, in that mixed models account for personality traits in addition to abilities. According to Cherniss (2010), personality traits can include constructs such as optimism, emotional self-efficacy, and selfesteem. Mixed model information is gleaned through self-report assessments, rather than performance-based measures.

Theoretical perspectives. From the conceptual framework and general models, three major theories of EI have emerged (Emmerling \& Goleman, 2003). Bar-On (2004) created the term emotional quotient (EQ), analogous to the IQ. His ability model is comprised of four components, including: 1) abilities to be aware of, understand, and express emotion; 2) abilities to understand and relate to others; 3) abilities to manage strong emotions and impulses; and 4) abilities to adapt and solve social problems. Within this model are five domains, which are as follows: "intrapersonal skills, interpersonal skills, adaptability, stress management, and general mood" (Bar-On, 2004, p. 116). In conjunction with his theory, Bar-On created an assessment by which to measure EI called the Emotional Quotient Inventory (EQI; Bar-On, 2004).

Mayer and Salovey (1997) took a different approach to theorizing EI. The pair suggested that EI is an ability by which we perceive emotions and access emotional content. Through this process, we generate emotions that assist our thought processes, which in turn aids in 
understanding emotions. From cultivating an understanding of emotions, we are better able to regulate them in a manner which promotes emotional and intellectual growth (Mayer \& Salovey, 1997). As Mayer and Salovey view EI as ability-based, the EQ is measured through standardized assessment in this theory. The measure developed to support this theory is the Mayer-SaloveyCaruso Emotional Intelligence Test (MSCEIT; Mayer, Salovey, Caruso, \& Sitarenios, 2003).

The third and most recent theory of EI was introduced by Goleman (1995). This theory suggests that EI occurs in four domains, including self-awareness, self-management, social awareness, and relationship management. According to Goleman, the aforementioned domains are foundational in the development of competency within that domain. In essence, individuals use emotional intelligence to learn and develop competencies related to themselves and others that improve their functioning. This theory differs from Bar-On's (2004) and Mayer and Salovey's (1997) in that it is competency based and was developed in the context of performance in the workplace (Goleman, 1995). The measure by which Goleman assesses EI is a selfassessment measure called the Emotional Competence Inventory (ECI) (Sala, 2002).

Measures and assessments. Within the two models of EI assessment are a multitude of measures. Ability-based assessments include tests such as Diagnostic Analysis of Nonverbal Accuracy Scales (Nowicki, \& Duke, 1994) and the Mayer-Salovey-Caruso Emotional Intelligence Tests (MSCEIT) (Mayer et al., 2003). These assessments are defined as abilitybased because they require task solving related to EI abilities in various domains. Self-report measures that would fall under the mixed model category are measures such as the Schutte SelfReport Emotional Intelligence Test (Schute et al., 1998) and the Emotional Quotient Inventory (EQ-i) (Bar-On, 1997). With the ability to measure EI, comes an opportunity to explore the utility of the construct in everyday life. 
EI has many practical applications and is relevant in every-day situations. Where mental health is concerned, EI scores on the MSCEIT were negatively correlated with depression, anxiety, and schizophrenia, meaning that higher EI scores indicate lower levels of mental health concerns (David, 2005). If higher EI is related to better mental health outcomes, it is no surprise that EI has a positive impact on various domains of life. In intimate partner relationships where both individuals are low on EI, poorer relational outcomes are likely to result (Brackett, Warner, \& Bosco, 2005). Similar findings exist among peer and familial relationships, suggesting that higher EI is associated with perceptions of supportive relationships (Lopes, et al, 2004). It can also be predictive of maladaptive choices such as drug and alcohol use and antisocial behaviors (Brackett, Mayer, \& Warner, 2004). It appears that EI plays an important role in helping individuals to effectively manage their internal experiences and social world, while also helping to direct behaviors.

Development and learning. Based on the existing literature, it appears that high levels of EI can have numerous benefits for individuals, both personally and interpersonally. This has led researchers to aim to understand if EI can be developed. Based on our knowledge regarding personality factors, it appears that there is a strong genetic influence on traits such as the Big 5 (Reimann, Angleitner, \& Strelau, 1997). McCrae and Costa (1991) developed the Big 5 personality traits, which are extraversion, agreeableness, conscientiousness, neuroticism, and openness. Further, research suggests that personality is constant through adulthood. Personality is not the only construct that is not malleable. Research suggests that IQ is relatively stable over time (Hewer, 2016). These factors have caused some doubt regarding the idea that EI can be developed over the lifespan. 
Despite the role that genetics appear to play in EI, it is important not to neglect the impact of nurture. Despite past research about the ability to develop EI, research suggests that EI can improve moderately. However, continuous effort and attention are required to make such improvements. Evidence supports the notion that training programs (Marrow, Jarrett, \& Rupinski, 1981; Nelis et al., 2009), psychotherapy (Barlow, 1985; Eack et al., 2007), and executive education (Boyatzis, Cowen, \& Kolb, 1995; Boytaz, Stubbs, \& Taylor, 2002) all improve EI. The field of neuroscience has also yielded promising results which may indicate that increases in EI are associated with changes in the brain. Davidson, Jackson, and Kalin (2000) found that plasticity exists in brain structures central in emotion. The outcomes of research in therapeutic, educational, and training settings, combined with advances in our knowledge of neuroscience, provide evidence that EI can likely be developed and learned.

Criticisms. One of the primary criticisms of EI is a general lack of consensus about the operational definition of the construct. Over time, many theorists have generated definitions and models of emotional intelligence. Among such theorists, none seem to agree on one, definitive description of the construct (Cherniss, 2010). In addition to this, EI is closely related to other concepts such as emotional and social competence, or personality traits. For the consumer, it becomes difficult to parse through the literature and come to an understanding of what EI is.

An additional criticism of EI is regarding measurement of the construct. There is concern that existing measures lack validity. There are numerous measures of EI, both ability-based and self-report. When reviewing the psychometric properties of the measures, limitations do exist. The context and criteria studied can cause fluctuations in the validity of EI measures (Cherniss, 2010). This may be especially relevant in the workplace. For example, work and academic performance are better predicted by IQ than EI (Van Rooy, \& Viswesvaran, 2004). However, EI 
is a better indicator of effective leadership, and interpersonal functioning (Goleman, 2002).

These differences in findings indicate that the context in which EI is studied affects the validity of the measures.

\section{Emotional Intelligence in the Workplace}

EI has been heavily researched in the context of work relations and productivity. The rationale for the importance of EI in the workplace is practical. Kjerulf (2014) hypothesized that EI is important for nine reasons. First, we use emotions to make decisions. Our emotions also guide our behavior within our working environments, affecting our relationships with others and our ability to engage in teamwork. His third hypothesis is that engagement and motivation are rooted in EI. Further, EI is related to creativity and innovative practices. Kjerulf (2014) also suggested that emotions are integral in the learning that takes place while we are on the job. The final four hypotheses regarding the importance of EI suggest that our emotions cannot be left at home. We carry them wherever we go. Additionally, it is important to note that stifled negative emotions gain strength, while stifled positive emotions grow increasingly weak. Finally, the expression of emotion means that individuals are invested in their workplaces.

EI in the workplace can be understood through two dimensions, focus and valence (Pekrun \& Frese, 1992). Focus is broken into two categories, task-related and social-related. These categories are also associated with valence, meaning the experience of positive or negative emotions within one of the focus categories. For example, within the task focus of a job, one could experience the valence of pride or enjoyment. Within the social-related focus, one could experience gratitude or admiration (Pekrun \& Frese, 1992).

Following these dimensions, research has identified many roles that EI plays in the workplace. A study by Salovey, Bedell, Detweiler, and Mayer (1999) found that individuals with 
higher EI are more effective at managing job related stress. Similarly, Slaski and Cartwright (2002) discovered that higher EI is associated with lower perceived stress in the workplace. Implications can also be seen in job performance. EI assesses an individual's performance at work with good predictive validity (Lopes, Salovey, Côté, \& Beers, 2005). EI is also linked to leadership in the workplace. Research suggests that EI is related to change-oriented leadership styles and intuition as well as effective leadership (Downey, Papageorgiou, \& Stough, 2006; George, 2000).

\section{Emotional Intelligence in Corrections}

There are various professions that require an individual to effectively manage personal stress and emotions while engaging in effective interactions with others (Denhof, Spinaris, \& Morton, 2014; Manzella \& West, 2003; Stys \& Brown, 2004). If the benefits of EI in the workplace are far reaching and impact most occupational domains, the value of the construct is without question. There are many identifiable occupations that are social in nature, are high stress, and require emotional fortitude. One such occupation is COs (Denhof, et al., 2014; Manzella \& West, 2003; Stys \& Brown, 2004). Higher levels of EI in corrections could facilitate effectiveness in this challenging work environment (Denhof, et al., 2014).

Literature regarding effective leadership in corrections parallels the abilities found in emotional intelligence (Newman, Guy, \& Mastracci, 2009). Goleman's (2006) explanation of this phenomenon is as follows, “The very best officers are adept at exercising influence, in the sense of constructively shaping the outcome of an interaction, using tact and self-control ... They approach volatile people with a professional demeanor, calmly and attentively" (p. 95). There are numerous situations in which a CO must display a degree of emotional intelligence. For example, COs must effectively communicate with offenders, and be able to interpret body 
language to prevent volatile situations (Stys \& Brown, 2004). As officers are responsible for the safety of their peers, themselves, the institution, and offenders, emotionally intelligent decision making is imperative. Manzella and West (2003) stated, "Officers with emotional intelligence will be much better equipped to successfully confront the challenges of their assignments when responding to demanding individuals and situations" (p. 85).

The benefits of EI have been seen in community corrections work as well. Andrews and Kiessling (1980) found that parole officers with high levels of empathy and social efficacy were more satisfied with their work roles, engaged in more prosocial dialogue, and produced probationers with lower recidivism rates and more respect for law enforcement. Ogińska-Bulik (2005) found that probation officers with higher emotional intelligence experienced lower perceived stress in the workplace, and had fewer negative health outcomes, specifically depressive symptoms. Examining emotional intelligence from an interpersonal lens, in the context of COs, may give us valuable insight into their understanding of relational factors that play a role in their correctional work environments, specifically as it relates to the offenders they encounter. Despite the seemingly important role of EI in correctional work, little research exists that examines the construct within this population.

\section{Emotional Intelligence in Related Fields}

Police officers have frequent interactions with the public, and encounter individuals from various walks of life. Because of their integrated role in communities, police officers experience significant levels of stress. The sources of officer stress are two-fold, including both organizational operations and the nature of police work (Martelli, Waters, \& Martelli, 1989). Organizational level stressors encompass issues related to lack of responsiveness and lack of support, while nature level stressors include issues such as danger, shift work, purpose, and 
frequently dealing with death and despair (Kroes, 1986; Ma, et al., 2015; Weber Brooks \& Leeper Piquero, 1998). Similar to work as a correctional officer, the nature of police work is subjectively stressful. Officers are required to fulfill their duties while maintaining effective interpersonal interactions.

Much like research on EI in corrections, there is a limited body of work that examines the role of EI in policing and law enforcement organizations. A study conducted by Al Ali, Garner, and Magadley (2012) found that higher EI enables officers to manage the emotions of themselves and others. The management of emotion promotes effective communication in their communities and within their agencies. This, in turn, helps to predict officer efficacy in other aspects of their job. This was further supported by Brunetto et al. (2012), who found that EI is predictive of perceived well-being and job satisfaction in officers. Further, these findings influenced officer engagement and lowered turnover plans. The authors suggested that, as EI has an impact on behavioral regulation, it can be beneficial in officer selection criteria, making EI just as valuable as knowledge of law and physical fitness.

Because of the distinct challenges associated with law enforcement, correctional officer work is a related occupation that may benefit from the promotion of EI (Stys \& Brown, 2004). In a setting where emotions have not historically been an integral part of the job, a shift in culture may be beneficial (Waldron \& Krone, 1991). A concerted focus on understanding and interpreting the emotions of self and others could create dynamic, lasting changes in the climate of correctional safety and security forces.

\section{Conclusions of the Literature}

An estimated 20 percent of individuals who are incarcerated in the United States suffer from mental illness. Institutions have made efforts to manage and assist this population in 
various ways, including the creation of acute mental health housing units. COs are tasked with ensuring the safety and security of facilities and are met with the unique challenge of managing offenders with mental illness. Because of the stressful nature of work in corrections, COs often experience significant stress and burnout. In recent decades, there has been a push to provide COs with mental health training to alleviate stress, increase knowledge of mental illness, and assist in the development of intervention skills.

Many forms of training include components of EI. The literature surrounding EI is vast. Conceptual and theoretical frameworks and models of the construct have been developed over the past 25 years and have yielded an abundance of research. Through this research, we have learned that EI has positive impacts on mental health, interpersonal relationships, lifestyle behaviors, and workplace performance, among other things. Based on our existing knowledge of EI in the workplace, the careers that meaningfully benefit from emotion regulation, interpersonal effectiveness, and stress management, are those that are interpersonal in nature. When utilized in correctional officer training, increased EI can yield positive outcomes. 


\section{CHAPTER 3: METHODS}

The attitudes and experiences of correctional officers (COs), as they pertain to interactions with offenders with mental illness, are not well understood at this time (Applebaum, Hickey \& Packer, 2001; Dvoskin \& Spiers, 2004; Galanek, 2015). As COs have frequent contact with affected offenders and play important roles in the treatment and management of mental illness within this population, the relationship between $\mathrm{CO}$ opinions about mental illness were of paramount concern in this study (Mauro, 2009; Sun, 2013).

The goal of this study was to identify correctional officer opinions about offenders with mental illness, and to understand the relationships among officer opinions, burnout, and emotional intelligence. Additionally, this study aimed to understand if mental health training for correctional officers led to higher emotional intelligence, more positive opinions, and lower levels of burnout. The Opinions about Mental Illness Scale (OMI) (Cohen \& Struening, 1962) was used to measure opinions, the Schutte Self-Report Emotional Intelligence Test (SSEIT) (Schutte, et al., 1998) was used to measure EI, and the Copenhagen Burnout Inventory (CBI) (Kristensen, Borritz, Villadsen, \& Christensen, 2005) was used to measure burnout. What follows is a review of the research questions that guided this study, and an in-depth discussion of the instruments that were used, and the process by which this research was conducted.

\section{Research Questions}

This in-depth, descriptive, correlational study surveyed correctional officers to examine the following research questions:

1. What is the relationship between correctional officer opinions about mentally ill offenders and emotional intelligence (EI), as measured by the Opinions about Mental Illness scale (OMI) and the Schutte Self-Report Emotional Intelligence Test (SSEIT)? 
2. Is there a significant relationship between correctional officer burnout, as measured by the CBI, and emotional intelligence, as measured by the SSEIT?

3. Does burnout moderate the relationship between opinions about mental illness and emotional intelligence?

4. Is there a significant relationship between exposure to mental health training and emotional intelligence as measured by the SSEIT?

5. When controlling for mental health training, is there a significant relationship between opinions about mental illness, as measured by the OMI, and emotional intelligence, as measured by the SSEIT?

6. Does exposure to mental health training moderate the relationship between opinions about mental illness and emotional intelligence in correctional officers?

\section{Participants}

For this study, eligible participants were individuals over the age of 18 , who were employed as correctional officers. Convenience sampling was used to identify participants. To estimate the sample size necessary for this quantitative study, an a priori power analysis was calculated using G*Power software (Faul, Erdfelder, Buchner, \& Lang, 2009). A moderated regression analysis was entered into $\mathrm{G}^{*}$ Power software with four predictor variables and one criterion variable. Based on an alpha $(\alpha)$ level of .05 , the power analysis calculated a minimum estimation of 77 participants to obtain statistical power of .80 and a medium effect size. The statistical power of .80 was selected for this study because of its prevalence within psychological research (Aron, Aron, \& Coups, 2014). An additional 50 participants were added to the sample size to increase statistical power and to account for the possibility of missing or incomplete data. The total estimated sample size was 127 participants. After collecting from 127 participants, the 
data was reviewed for missing and incomplete data. Missing and incomplete data were removed from the sample, leaving a total of 105 completed surveys. The total sample size in the study was $105(n=105)$.

\section{Measures}

Opinions about Mental Illness Scale (OMI; Cohen, \& Struening, 1962). The OMI scale (Appendix G) was developed in 1959 to assess opinions that healthcare workers maintained regarding mental illness. Responses to the OMI scale indicate attitudes about etiology, treatment, and prognosis of psychiatric disorders. The OMI scale consists of 51 items in the form of opinion statements. Items are on a 6-point Likert-type scale, and responses range from 1 (strongly agree) to 6 (strongly disagree). The scale also consists of five subscales which are as follows:

1. Factor A: authoritarianism (11 items). High scores indicate the opinion that individuals with mental illness are dangerous, should be controlled by society.

2. Factor B: unsophisticated benevolence (14 items). High scores refer to opinions that are paternal. Mental illness is viewed as a misfortune.

3. Factor C: mental hygiene ideology (9 items). High scores refer to a treatment orientation and the idea that mental illness is similar to physical illness and should receive specialized treatment.

4. Factor D: social restrictiveness (10 items). High scores refer to the attitude that individuals with mental illness should have restrictions in some social domains for the protection of society.

5. Factor E: interpersonal etiology (9 items). High scores refer to the idea that mental illness is the result of problematic interpersonal relationships, and the recognition of life experiences in the development of abnormal behavior. 
Factors A and D are negatively worded, where higher scores represent more negative opinions about mental illness, and Factors B, C, and E are positively worded, where higher scores represent more positive opinions about mental illness. The construct validity of the OMI scale was established using a 200 -item pool. Items were analyzed by over 8,000 mental health professionals. The final 51-item version of the scale was selected after extensive factor analysis. Higher scores on subscales indicate a stronger endorsement of the dimension. The OMI scale has sound psychometric properties. The scale shows acceptable internal consistency reliability, with values between .65 and .80 on authoritarianism (.77), unsophisticated benevolence (.70), social restrictiveness (.71), and interpersonal etiology (.65). Factor C, mental hygiene ideology, showed low reliability (.39). Extensive factor analytic studies have been conducted by Cohen and Struening $(1962 ; 1963)$ to establish evidence of validity.

The OMI scale was selected for use in this study because of its sound psychometric properties, its scope of coverage of prominent domains of stigma, and its accessibility. The OMI is free for public use and no permissions are required. Additionally, the scale has been used extensively in research of opinions of mental illness for nearly 60 years in populations including a broad range of hospital personnel, police officers, college students, and the general public (Cohen \& Struening, 1962; Drolen, 1993; Kazantzis et al., 2009).

For this study, a modified version of the scale was used. Modification was chosen for several reasons, including to decrease the amount of time that participants would spend completing survey measures and to eliminate Factor C, as it shows poor reliability. Additionally, previous literature suggests that COs maintain negative attitudes toward offenders with mental illness. Because of this finding, and for the purpose of this study, participants only completed negatively worded items, Factor A: Authoritarianism and Factor D: Social Restrictiveness. The 
modified version of the scale was comprised of 21 items. These scales are negatively worded, where higher scores represent more negative opinions about mental illness. Prior to beginning analysis, a Pearson Correlation was conducted to determine if OMI subscales Factor A:

Authoritarianism and Factor D: Social restrictiveness were correlated. The scales consisted of 21 items and showed an acceptable level of internal consistency, as determined by a Cronbach's alpha of .77. As the subscales were found to be correlated, scores on these items of the OMI were averaged to create a single, composite score.

Schutte Self-Report Emotional Intelligence Test (SSEIT; Schutte, et al., 1998). The SSEIT (Appendix H) was developed to measure emotional intelligence, as conceptualized by Salovey and Mayer (1990). The SSEIT is a 33-item self-report measure that produces scores in three categories: appraisal and expression of emotion in the self and others, regulation of emotion in the self and others and utilization of emotions in solving problems. Items are scored on a 5 point, Likert-type scale, ranging from 1 (strongly disagree) to 5 (strongly agree). Items 5 , 28, and 33 are reverse scored. Each sub-test score is then added together to give a total score for participants. Scores can range from 33-165, with higher scores indicating more emotional intelligence. The SSEIT is individually administered and is written at a fifth-grade reading level.

The SSEIT was originally normed on 346 participants and showed good internal consistency reliability $(a=.90)$. To confirm these findings of internal consistency, a cross-check of 32 college students was conducted and yielded a Cronbach's alpha of 0.87. Subsequent studies have shown internal consistency reliability ranging from .70 to .85 (Jonker \& Vosloo, 2008; Petrides \& Furnham, 2000). Two-week test-retest reliability indicated that the scores were fairly stable over time $(r=.78)$. 
The SSEIT has also been tested for correlation with theoretically related constructs.

Higher scores on the SSEIT were found to be associated with less alexithymia, increased attention to feelings, greater optimism, less pessimism, less depression, and less impulsivity. The SSEIT has been used in many studies, including with populations such as adolescents, university students, human service professionals, and customer service employees (Abraham, 2000; Austin, Saklofske, Huang, \& McKenney, 2004; Ciarrochi, Chan, \& Bajgar, 2000; Oginska-Bulk, 2005). The SSEIT was used as a measure of global, trait EI. The scale was chosen for inclusion in this study due to its sound psychometric properties, its brevity, and its accessibility. The SSEIT is free for public use, and no permissions are required.

Copenhagen Burnout Inventory (CBI; Kristensen, Borritz, Villadsen, \& Christensen, 2005). The CBI (Appendix I) is a 19-item self-report measure. It contains items that are both positively and negatively worded, covering three areas which are as follows: personal, work and client-related, where personal refers to the degree of physical and psychological fatigue, work refers to degree of physical and psychological fatigue at work, and client-related refers to client, patient, student, etc., burnout. Physical and psychological fatigue is composed of six items, work-related fatigue consists of seven items, and client-related burnout consists of six items. Responses to scale items are scored on a 5 point, Likert-type scale. The response options are recorded into scores of 0-100 (100 = always/ to a very high degree, $75=$ often/to a high degree, $50=$ sometimes/somewhat, $25=$ seldom/to a low degree, $0=$ never/to a very low degree). Scores are then averaged. The CBI does not have cutoff scores. However, higher scores indicate higher levels of burnout.

The CBI was normed in a larger study, the Project on Burnout, Motivation, and Job Satisfaction (PUMA) (Kristensen et al., 2005). The PUMA study was a five-year study of 1,914 
employees working in human service sectors. Participants included employees at a state psychiatric prison, employees at a social welfare office, homecare service providers, and employees at psychiatric hospitals.

As part of the PUMA study, the CBI was assessed for both reliability and validity. Internal consistency scores from 0.85 to 0.87 were found. Validity was demonstrated through correlations with the Short Form 36 instrument (-0.75). When compared to the Maslach Burnout Inventory (MBI), the CBI was found to be equal in determining burnout levels. There are also correlations between the CBI at baseline and three-year follow-up. Correlations above .50 were found between the first and second administration. It is important to note that burnout levels may change over time. Subsequent studies have found that higher CBI scores predicted absence due to sickness, intent to resign, and poor sleep hygiene (Borritz et al., 2006; Hoigaard, Giske, \& Sundsli, 2012; Toker \& Melamed, 2017).

The CBI originated in Denmark. Since its inception, the scale has been translated into eight languages, including English. It was chosen for inclusion in this study due to its sound psychometric properties, its use within various human service sectors, and its accessibility. The CBI is free for public use, and no permissions are required.

Demographic Questionnaire. A five-item demographic questionnaire (Appendix F) was given to participants. Participants were asked their age and gender. They were also asked if they are currently employed as a correctional officer, their length of employment as a correctional officer, and how many hours of mental health training they have completed. Demographic information is reported in Tables 1, 1.1, and 1.2. Additionally, hours of mental health training served as both an independent variable and moderating variable during analyses. 


\section{Procedures}

Approval to collect data for the study was acquired from the West Virginia University (WVU) Institutional Review Board (IRB) (Appendix J). After approval was obtained, an electronic message containing a cover letter (Appendix B) and the link to a survey including Informed Consent (Appendix A), and the randomly counter-balanced OMI, SSEIT, CBI (Appendices G, H, and I), and demographic questionnaire (Appendix F) was disseminated to closed correctional officer groups on Social Media Platforms, including Facebook and LinkedIn, notifying them of the opportunity to voluntarily participate in the study. The instruments were randomly delivered to address order effects. The survey was created through WVU Qualtrics survey software.

The content of social media posts followed Dillman's (1978) recommendations for creating a well-composed letter. Per Dillman's (1978) recommendations, the post provided prospective participants with the usefulness, significance, purpose, risks and benefits of the study. Additionally, the informed consent alerted them to their right to exit the study at any point, of the confidential nature of their responses, that their participation was voluntary, and that the researcher was conducting the study in conjunction with a doctoral dissertation. A reminder post was created two weeks after the original contact for recruitment (Appendix C). A third and final reminder post was created two weeks following the second contact (Appendix D).

After completion of the OMI, SSEIT, CBI (Appendices G, H and I), and demographic questionnaire (Appendix F), participants were provided with post-study information. The post study information form (Appendix E) thanked participants for completing the study and included an anonymity and confidentiality statement. While adverse effects related to participation were not expected, the post-study information included services for participants who may have 
experienced distress following involvement in the study. Participants were also provided with researcher contact information in the event that they had questions regarding the results of the study.

Participants who completed the questionnaire then received information about the opportunity to enter a drawing to receive one of 120, \$10 Amazon gift cards. Participants who indicated that they wished to enter the drawing were redirected to a distinct link, separate from the survey, to ensure anonymity and privacy. They were prompted to enter their email address at that time. Participants who did not wish to enter the drawing were thanked for their participation and prompted to close the window. Once finished, participants had no further contact with the researcher, with the exception of those who chose to participate in the gift card incentive. There were 127 initial participants in the study. After accounting for missing and incomplete data, there were 105 participants. Each participant who completed the survey and provided an email address received an email with an electronic link to the Amazon gift card.

\section{Design}

The quantitative study utilized a cross sectional design and was descriptive and correlational. Survey measures were used to collect data from participants. Participants completed the SSEIT, OMI, CBI, and a demographic questionnaire. All participants were asked to complete all measures, and measures were randomly counterbalanced to address order effects. Campbell and colleagues (1981) recommended that a pilot test should include 12 to 50 participants. Per their recommendations, the survey was piloted on 12 participants. Following a pilot test of the survey, it was estimated that the survey would take approximately 20 minutes to complete. 


\section{Data Analysis and Hypotheses}

Data were analyzed using Statistical Package for Social Sciences (SPSS) software.

Descriptive statistics were calculated and reported in Table 1. Data was assessed for normality prior to conducting the analyses. Data and analyses were stored on an encrypted flash drive to ensure safety and confidentiality. Each hypothesis and its respective analysis strategy are described below:

Hypothesis 1: Higher, more negative attitude scores on the OMI will predict lower emotional intelligence scores (SSEIT). A simultaneous regression was used to test this hypothesis.

Hypothesis 2: Higher burnout scores on the CBI will predict lower emotional intelligence scores (SSEIT). A simultaneous regression was used to test this hypothesis.

Hypothesis 3: Burnout (CBI) scores will moderate the relationship between opinion (OMI) scores and emotional intelligence (SSEIT) scores such that when the moderator is present, there will be a significantly stronger relationship between emotional intelligence and opinions. A moderated regression was used to test this hypothesis.

Hypothesis 4: Greater exposure to mental health training will predict higher emotional intelligence (SSEIT). A simultaneous regression was used to test this hypothesis.

Hypothesis 5: When controlling for mental health training, higher, more negative opinions about mental illness (OMI) scores will predict lower emotional intelligence (SSEIT) scores. A simultaneous regression was used to test this hypothesis.

Hypothesis 6: Exposure to mental health training will moderate the relationship between opinion scores (OMI) and emotional intelligence (SSEIT), such that when the moderator is 
present, there will be a significantly stronger relationship between emotional intelligence and opinions about mental illness. A moderated regression was used to test this hypothesis.

\section{Summary}

This study sought to better understand the relationships among emotional intelligence, burnout, mental health training, and correctional officer opinions about offenders with mental illness. The study was a descriptive, correlational survey design, with solicited participants who are employed as correctional officers in state prisons, regional and county jails, and federal prisons. Participants were selected through convenience sampling and were recruited through social media outlets. Participants in the study were asked to provide demographic information, complete a measure of opinions of mental illness, complete a measure of burnout, and complete a measure of emotional intelligence. A pilot test of the survey indicated that the estimated time to complete the survey was approximately 20 minutes. Participants were provided with post-study information and given the opportunity to choose to enter a drawing for an Amazon gift card. Data was analyzed using SPSS software. The aim of the study was to add to the existing literature regarding $\mathrm{CO}$ experiences, as they relate to mentally ill offenders. Additionally, it aimed to assist in the development and implementation of training opportunities and policies within correctional settings. 


\section{CHAPTER 4: RESULTS}

Existing literature suggests that correctional officers have high levels of burnout and generally maintain negative opinions about inmates with mental illness (Applebaum, Hickey \& Packer, 2001; Dvoskin \& Spiers, 2004; Galanek, 2015). Mental health training that includes components of emotional intelligence has shown to be effective in decreasing burnout and improving perceptions of inmates with mental illness (Andrews \& Kiessling, 1980; Manzella \& West, 2003; Stys \& Brown, 2004). The goal of this study was to expand the literature by analyzing the relationships among the identified factors of correctional officer opinions about mental illness, burnout, and emotional intelligence. Additionally, burnout and hours of mental health training were used as moderating variables in the study.

\section{Preliminary Analyses}

Participant descriptive statistics were calculated and can be found in Table 1. Upon initial data collection, there were 127 participants in the study. After accounting for missing and incomplete data, there were a total of 105 completed surveys. Of the sample, $65 \%$ were male and $35 \%$ were female. Additionally, $88 \%$ were employed in State Prisons, and Regional and County Jails, while the remaining $12 \%$ were employed in Federal Correctional Facilities. The average age of participants was $40(\mathrm{SD}=10.2$, Range $=18-63)$. On average, participants were employed as correctional officers for 11 years $(\mathrm{SD}=7.4$, Range $=1-30)$ and completed 22 hours of mental health training $(\mathrm{SD}=27.6$, Range $=0-110)$, separate from pre-service training.

A Pearson bivariate correlation matrix was created to identify possible relationships between the primary variables in the study and participant demographics. Of note, the strength of correlational relationships was established by Aaron, Aaron, and Coups (2014). Aaron and colleagues reported that correlation coefficient effect sizes are as follows: Small $r=.10$, Medium 
$r=.30$, and Large $r=.50$ (2014). The results of the bivariate correlation matrix are seen in Table 2.

As seen in Table 2, during preliminary analyses, the six primary hypotheses in the study are not supported. Although there are no significant results in the preliminary correlational analyses that support the hypotheses in the study, interesting relationships between variables do exist. Results indicate that Burnout, as measured by the CBI, and Emotional Intelligence, as measured by the SSEIT, have a significant, small, positive association. This finding contrasts with Hypothesis 2 and suggests that higher levels of burnout are associated with higher levels of emotional intelligence $(r=.19, p<.05)$. This finding will be further addressed in the discussion. Additionally, results indicate that there is a significant, small, positive association between Opinions About Mental Illness (OMI) and age, $(r=.19, p<.05)$, indicating that older participants endorsed more negative opinions about mental illness. This may be of interest in future or exploratory studies.

Table 1

Correctional Officer Descriptive Statistics

\begin{tabular}{lccccc}
\hline Variable & N & $\begin{array}{c}\text { Sample } \\
\text { Mean }\end{array}$ & SD & Range & $\begin{array}{c}\text { Cumulative } \\
\text { Percent }\end{array}$ \\
\hline 1. Age & 105 & 40.68 & 10.23 & $18-63$ & -- \\
2. MHtrn & 105 & 22.22 & 27.61 & $0-110$ & -- \\
3. EmpLen & 105 & 10.86 & 7.45 & $1-30$ & -- \\
4. Gender: Male & 68 & -- & -- & -- & 64.8 \\
& & & & -- & 35.2
\end{tabular}


6. Facility: State

92

Prison/ Regional Jail/

County Jail

7. Facility: Federal

Prison

Note. $N=105 ;$ MHtrn $=$ Hours of Mental Health Training; EmpLen = Employment Length .

Table 2

Bivariate Correlation Matrix: Correlations Among

Correctional Officer Demographic Variables

\begin{tabular}{lcc}
\hline Variable & CBI & OMI \\
\hline 1. SSEIT & $.196^{*}$ & .084 \\
2. Mental Health Training & .032 & .249 \\
3. Employment Length & -.139 & .104 \\
4. Age & .005 & $.193^{*}$
\end{tabular}

Note. $n=105 ;$ SSEIT = Schutte Self-Report Emotional Intelligence Test; Mental Health Training = Hours of Mental Health Training; CBI = Copenhagen Burnout Inventory; OMI = Opinions About Mental Illness Scale.

$* p<.05$

\section{Primary Analyses}

The following section outlines the major findings for the hypotheses in the study. For this study, the research questions were analyzed in two distinct blocks. Research questions one, two, and three were analyzed in a series of regression models, as each research question builds on the previous question to determine if $\mathrm{OMI}, \mathrm{CBI}$, and the interaction term predict emotional intelligence. 
Correspondingly, research questions four, five, and six were analyzed in a series of regression models, as each research question builds on the previous question to determine if OMI, hours of mental health training, and the interaction term predict emotional intelligence. The statistical analyses and relevant findings of the analyses are discussed in the following sections.

\section{CBI as a Moderator of OMI and SSEIT}

For this study, a modified version of the Opinions About Mental Illness (OMI) scale was chosen to decrease the amount of time that participants would spend completing survey measures and to eliminate Factor C: Mental Hygiene Ideology, as it has shown poor reliability. Additionally, previous literature suggests that COs maintain negative attitudes toward offenders with mental illness. Because of this finding, and for the purpose of this study, participants only completed negatively worded items, Factor A: Authoritarianism and Factor D: Social Restrictiveness. The modified version of the scale was comprised of 21 items. These items are negatively worded, where higher scores represent more negative opinions about mental illness. Prior to beginning analysis of hypothesis one, a Pearson Correlation was conducted to determine if OMI subscales Factor A: Authoritarianism and Factor D: Social restrictiveness were correlated. The scales consisted of 21 items and showed an acceptable level of internal consistency, as determined by a Cronbach's alpha of .77. As the subscales were found to be correlated, scores on these items of the OMI were averaged to create a single, composite score. 
Table 3

Summary of Multiple Regression Analyses for Variables Predicting Emotional Intelligence: Burnout as a Moderating Variable

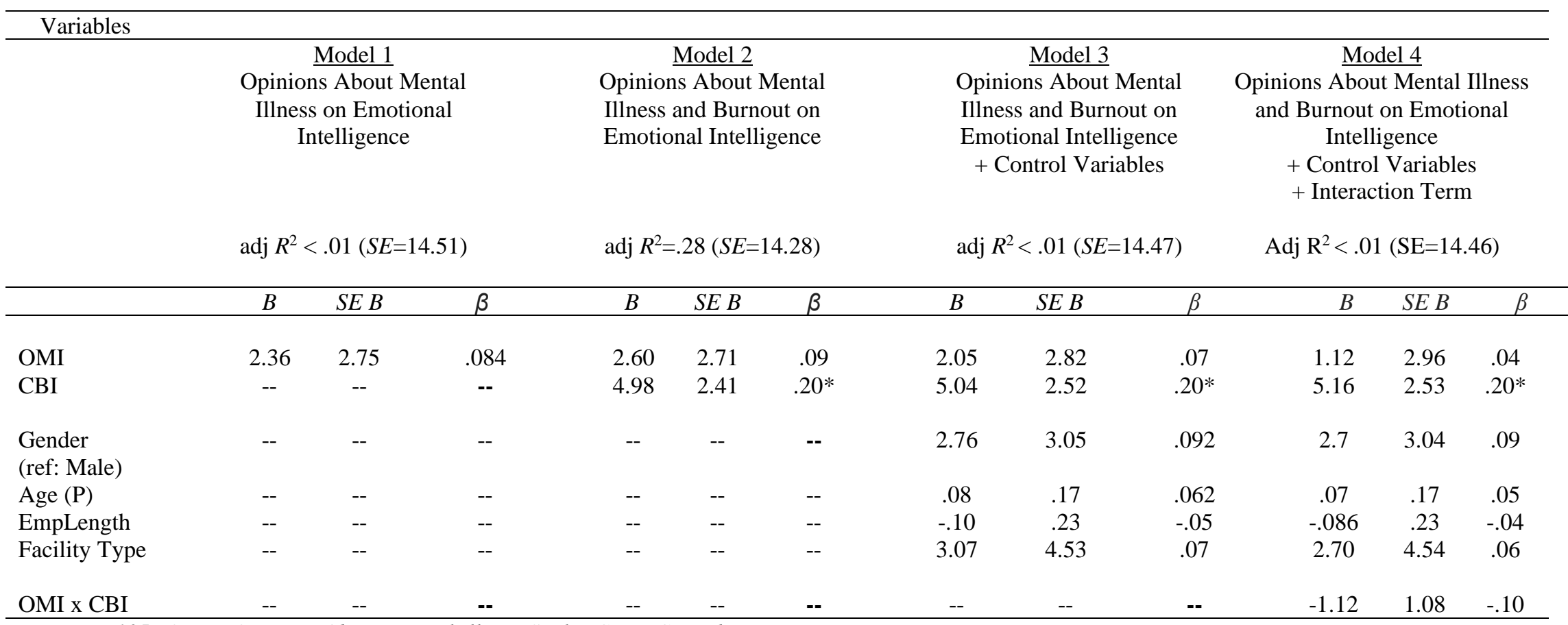

Note. $N=105 ;$ OMI = Opinions About Mental Illness Scale; CBI= Copenhagen Burnout Inventory;

EmpLength=Employment Length.

$* p<.05 ; * * p<.01$ 
Hypothesis one states that higher attitude scores (OMI) will predict lower emotional intelligence scores (SSEIT). A linear regression was used to test this hypothesis (Table 3, Model 1). In Model 1, the predictor variable OMI was regressed onto the SSEIT outcome variable using the regression equation SSEIT $=112.406+(2.361 \times$ OMI $)$. Model 1 suggests that opinions about mental illness do not significantly predict levels of emotional intelligence $F(1,103)=.733, p=$ .394. These findings do not support hypothesis one.

Hypothesis two states that higher burnout scores (CBI) will predict lower emotional intelligence scores (SSEIT). This was addressed using a linear regression analysis and can be found in Model 2 of the regression analysis in Table 3. The predictor variables CBI and OMI were regressed onto the SSEIT outcome variable. Results from the analysis suggest that, when accounting for opinions about mental illness (OMI) scores, burnout (CBI) independently predicts emotional intelligence $F(2,102)=2.509, p<.05$. There is a significant, positive relationship between the variables, suggesting that, as levels of burnout increase, levels of emotional intelligence also increase. This was also found during preliminary analysis $(r=.196, p<.05)$. These findings suggest the inverse of hypothesis two is true, therefore not supporting the proposed hypothesis.

Control variables were added into the model to account for participant demographic variables (Table 3, Model 3). Results suggest that burnout remained a significant predictor in the model when controlling for demographic variables $\mathrm{F}(6,98)=1.05, p=.04$. Among the demographic variables, none were found to statistically significantly predict emotional intelligence.

Hypothesis three states that burnout will moderate the relationship between opinions and emotional intelligence, such that when the moderator is present, there will be a significantly 
stronger relationship between emotional intelligence and attitudes. A moderated regression analysis was used to test this hypothesis. The interaction term (OMI x CBI) was included in the model to determine if burnout is a moderating variable in the relationship between opinions about mental illness and emotional intelligence (Table 3, Model 4). In the model, the interaction term was not found to be a significant predictor, indicating that burnout does not moderate the relationship between opinions about mental illness and emotional intelligence $\mathrm{F}(7,97)=1.05, p$ $=.30$.

\section{Mental Health Training as a Moderator of OMI and SSEIT}

Hypothesis four states that more hours of mental health training will predict higher levels of emotional intelligence (SSEIT). A linear regression was used to test this hypothesis (Table 4, Model 1). In Model 1, the predictor variable, mental health training, was regressed onto the SSEIT outcome variable using the regression equation SSEIT $=112.406+(2.361 \mathrm{x}$ OMI $)$. Model 1 suggests that hours of mental health training do not significantly predict levels of emotional intelligence $F(1,103)=.733, p=.394$. These findings do not support hypothesis four.

Hypothesis five states that, when controlling for hours of mental health training, opinions of mental illness (OMI) will predict higher levels of emotional intelligence (SSEIT). A linear regression analysis was used to test this hypothesis and is outlined in Table 4, Model 2. The predictor variables Mental Health Training and OMI were regressed onto the SSEIT outcome variable. Results from the analysis indicate that, when accounting for Mental Health Training scores, OMI does not predict emotional intelligence $F(2,102)=1.847, p=.51$. These findings do not support hypothesis five.

The control variables in the study were added into the model to account for participant demographic variables (Table 4, Model 3). Results suggest that mental health training and OMI 
remained non-significant predictors of SSEIT in the model, when controlling for demographic variables. Among the demographic variables, none were found to statistically significantly predict emotional intelligence.

Hypothesis six states that mental health training will moderate the relationship between attitude scores (OMI) and emotional intelligence (SSEIT), such that when the moderator is present, there will be a significantly stronger relationship between emotional intelligence and opinions about mental illness. A moderated regression was used to test this hypothesis. The interaction term (OMI x Mental Health Training) was included in the model to determine if Mental Health training is a moderating variable in the relationship between opinions about mental illness and emotional intelligence (Table 4, Model 4). In the model, the interaction term was not found to be a significant predictor, indicating that mental health training does not moderate the relationship between opinions about mental illness and emotional intelligence $F(7$, 97) $=.86, p=.93$. 
Table 4

Summary of Multiple Regression Analyses for Variables Predicting Emotional Intelligence: Mental Health Training as a Moderating Variable

\begin{tabular}{|c|c|c|c|c|c|c|c|c|c|c|c|c|}
\hline \multicolumn{13}{|l|}{ Variables } \\
\hline & \multicolumn{3}{|c|}{$\begin{array}{l}\text { Model } 1 \\
\text { Mental Health Training on } \\
\text { Emotional Intelligence }\end{array}$} & \multicolumn{3}{|c|}{$\begin{array}{l}\text { Model } 2 \\
\text { Mental Health Training and } \\
\text { Opinions About Mental Illness } \\
\text { on Emotional Intelligence }\end{array}$} & \multicolumn{3}{|c|}{$\begin{array}{c}\text { Model } 3 \\
\text { Mental Health Training and } \\
\text { Opinions About Mental } \\
\text { Illness on Emotional } \\
\text { Intelligence } \\
\text { + Control Variables }\end{array}$} & \multicolumn{3}{|c|}{$\begin{array}{c}\text { Model 4 } \\
\text { Mental Health Training and } \\
\text { Opinions About Mental Illness } \\
\text { on Emotional Intelligence } \\
\text { + Control Variables } \\
\text { + Interaction Term }\end{array}$} \\
\hline & \multicolumn{3}{|c|}{$\operatorname{adj} R^{2}=.02(S E=14.33)$} & \multicolumn{3}{|c|}{$\operatorname{adj} R^{2}=.01(S E=14.37)$} & \multicolumn{3}{|c|}{$\operatorname{adj} R^{2}<.01(S E=14.49)$} & \multicolumn{3}{|c|}{$\operatorname{adj} R^{2}<0.1(S E=14.56)$} \\
\hline & $B$ & $S E B$ & $\beta$ & $B$ & $S E B$ & $\beta$ & $B$ & $S E B$ & $\beta$ & $B$ & $S E B$ & $\beta$ \\
\hline MH Training & .09 & .05 & .17 & .08 & .05 & .16 & .10 & .05 & .19 & .10 & .05 & .20 \\
\hline OMI & -- & -- & -- & 1.82 & 2.74 & .06 & 1.20 & 2.84 & .04 & 1.18 & 2.87 & .04 \\
\hline $\begin{array}{l}\text { Gender } \\
\text { (ref: Male) }\end{array}$ & -- & -- & -- & -- & -- & -- & 1.91 & 3.01 & .06 & 1.91 & 3.03 & .06 \\
\hline Age $(\mathrm{P})$ & -- & -- & -- & -- & -- & -- & .17 & .17 & .12 & .17 & .17 & .12 \\
\hline EmpLength & -- & -- & -- & -- & -- & -- & -.31 & .24 & -.16 & -.31 & .24 & -.16 \\
\hline Facility Type & -- & -- & -- & -- & -- & -- & 3.94 & 4.51 & .09 & 3.87 & 4.61 & .08 \\
\hline $\begin{array}{l}\text { MH Training } \mathrm{x} \\
\text { OMI }\end{array}$ & -- & -- & -- & -- & -- & -- & -- & -- & -- & -.14 & 1.75 & -.00 \\
\hline
\end{tabular}

Note. $N=105 ;$ MH Training = Mental Health Training; OMI = Opinions About Mental Illness Scale; EmpLength =

Employment Length

$* p<.05 ; * * p<.01$ 


\section{Summary}

The findings indicate that there are no statistically significant relationships between correctional officer opinions of mental illness and emotional intelligence, or mental health training and emotional intelligence. The results also suggest that burnout does not moderate the relationship between opinions of mental illness and emotional intelligence. Likewise, mental health training was not found to moderate the relationship between opinions of mental illness and emotional intelligence. Of the six hypotheses, the only significant finding was that there is a positive relationship between burnout and emotional intelligence. These surprising results suggest that, as levels of burnout increase, levels of emotional intelligence also increase, which was the inverse of hypothesis two. 


\section{CHAPTER 5: DISCUSSION}

\section{Discussion of Participant Demographics}

Results suggest that the sample acquired for the study differs somewhat from the reported general population of correctional officers in the United States but is consistent with employment trends. In 2008, women accounted for only $14 \%$ of correctional officers across the United States (Winters, 2014). Statistics from 2020 indicate that women now account for approximately $28 \%$ of correctional officers (Federal Bureau of Prisons, 2020). The American Correctional Association suggested there has been a $40 \%$ increase in female correctional officers over the previous eight years (Gibson, 2018; MTC institute, 2017). In the present study, 35\% of the sample were female correctional officers (Table 1). This is consistent with the trend toward more women seeking employment in correctional settings. This study used social media resources to recruit participants. Based on the percentage of women in the current sample, this population may be more inclined to seek membership in online supportive groups for correctional officers and may be more accessible through social media outlets than their male counterparts. Given this study's finding, it is possible that the profession is again seeing a growth in the number of female COs. Therefore, future research would benefit from targeting the perspectives of female officers.

Consistent with nationwide trends, the study's sample is comprised primarily of COs working in state prisons and regional and county jails. Of COs surveyed in the study, $92 \%$ were employed in state prisons, regional jails, or county jails, and $8 \%$ were employed in Federal Prisons (Table 1). Statistics from 2016 indicate that federal prisons account for only $10 \%$ of national correctional institutions, with the remaining $90 \%$ being operated by states (Bureau of Justice Statistics, 2016; Sawyer \& Wagner, 2020). Inmates with mental illness are most likely to be housed in regional and county jails, and account for $20 \%$ of their general prison populations 
(Kaeble et al., 2016; Steadman et al., 2009; Torrey et al., 2014). Working with inmates with mental illness may be especially common for officers working in jail settings. Jail COs are often the first to recognize inmate behavioral change related to mental illness. They are instrumental in alerting mental health staff to such changes, making it important that these officers have fundamental training in recognizing the signs and symptoms of mental illness.

The average age of participants in the study was $40(\mathrm{SD}=10.22)$ (Table 1). This is consistent with the general correctional officer population whose average age for males is 39.9 and is 40.2 for females (Data USA, 2017). Data on correctional officer employment was also gathered during the study and revealed an average length of employment of 10 years, with a standard deviation of 7.45 (Table 1). The personal and professional toll of employment as a correctional officer has been discussed at length and includes demanding and stressful work and the potential risks to individual health and safety (Cullen et al., 1985; Liebling, Price, \& Shefer, 2011; Spinaris et al., 2012). COs have the highest turnover rate of all law enforcement employees (Isele, 2020) and have one of the highest rates of illness and injury of any profession (Kaufman, 2019). Given the demands and potential negative consequences of the job, state and federal correctional facilities often experience high rates of turnover and officers often do not remain in their positions long-term (Fifield, 2016; Kaufman, 2019). In the present study, many of the officers were longer-term career veterans, differing somewhat from the general population of correctional officers. Given that participants were recruited from $\mathrm{CO}$ supportive groups on social media, the individuals surveyed may feel a stronger connection to their professional identity and have a greater inclination to maintain supportive contact with their fellow officers. This could serve as a potential protective factor in these officers and provide some explanation for lowering turn-over and their longevity of employment. 
Within the study, information regarding hours of completed mental health training, separate from pre-service training, was obtained from COs. Hours of mental health training was used as both a predictor and moderating variable in the study. Officers were asked to self-report the total number of hours of mental health training they had acquired over their tenure as a CO. Participants reported an average of 22.22 hours of mental health training (Table 1). In this figure, the standard deviation is 27.61 , suggesting considerable variability among completed hours. Variation among the sample is expected, given that state prisons, regional jails and county jails do not have a national standard of providing mental health training to which they adhere, and 92 percent of the sample work in these settings (Dvoskin \& Spiers, 2004; Kois, Hill, Gonzales, Hunter, \& Chauhan, 2020).

There are, however, other areas of concern with these self-reported data. In the federal system, COs across institutions and regardless of location, are required to complete the same training, including mental health training. Within the sample, officers in the federal system, having worked similar lengths of time, reported a vastly different number of acquired hours of mental health training. For example, one participant employed as a federal $\mathrm{CO}$ for 15 years reported 100 total hours of mental health training. Another participant who was employed for 13 years as a federal $\mathrm{CO}$ reported zero hours of mental health training. This same pattern of discrepancy was seen throughout the participants employed in federal facilities. The question regarding hours of mental health training was problematic, which is supported by the inconsistencies found within the data. This could be for a several reasons. Participants may have misunderstood the information the question attempted to gather, or participants may have attempted to make an educated guess regarding total hours of training if they were unsure. 
Regardless, the data gathered on this demographic item may not be an accurate representation of actual mental health training.

Conversely, it is also possible that officers did accurately report their completed hours of mental health training. This would be problematic for several reasons. If officers are required to complete an established number of hours of mental health training, and the hours are not being offered or completed, COs are left to manage difficult inmates with little to no education and training. COs should have, at minimum, a baseline understanding of mental illness and how it can impact inmate behavior. Without some foundational knowledge, COs are likely ill prepared to manage and intervene with inmates who are suffering from mental illness. It is a disservice to both affected officers and the inmates with whom they work if appropriate training is not provided.

\section{Discussion of Preliminary Analyses}

The findings of the preliminary analyses indicate that, on average, COs in the study maintained negative opinions about offenders with mental illness. Officer mean OMI scores were 4.27 on a 6-point scale $(\mathrm{SD}=.51$, Range=3.00-5.38), indicating that $\mathrm{COs}$ tend to be of the opinion that individuals with mental illness are dangerous and should be controlled by society and that individuals with mental illness should have restrictions in some social domains for the protection of society. This is consistent with previous research that found COs maintain negative attitudes toward inmates with mental illness, often viewing them as dangerous and unpredictable (Callahan, 2004; Harmon-Nary, 2013; Keeler, 2017; Kropp, et al., 1989).

In the present study, a significant, small, positive correlation was found between OMI scores and age, suggesting that older correctional officers maintained more negative opinions about inmates with mental illness than their younger counterparts. There are a few possible 
explanations for this relationship. It may be reflective of the general changes in the current culture's perceptions of mental illness that have occurred over time. Research suggests that the general population of older adults have poorer mental health literacy, more negative attitudes about depression, and more negative attitudes about mental health care, as compared to younger adults (Connery \& Davidson, 2006; Hasan \& Link, 1988; Robb, et al., 2003; Segal, et al., 2005). There was also an expected, significant positive correlation between age and length of employment. Another possible explanation for the relationship between OMI and age could be that, as correctional officers gain more exposure to mentally ill inmates, they develop more negative opinions about the population. Previous research has found that negative opinions are associated with concerning standpoints, including that mental health services are a waste of money, that coercion should be used in treatment, and that mentally ill inmates are more violent and dangerous than the general inmate population (Callahan, 2004; Keeler, 2017).

Kristenson and colleagues (2005) normed the CBI on over 1,900 participants working in various settings including a state prison, social welfare offices, psychiatric hospitals, homecare, and institutions for the severely disabled in Denmark. The average scores for the COs in the norming sample for the CBI are consistent with the average scores of COs in the present study. COs endorsed moderate levels of burnout, most notably client-related burnout $(\mathrm{M}=3.02, \mathrm{SD}=$ .58 , Range $=1.58-4.42$ ). Despite reporting some symptoms of burnout, officers did not endorse high levels of burnout across personal, work-related, and client-related domains. Previous studies surveying COs in the United States have found that officers report high levels of stress and symptoms of burnout (Lambert, Cluse-Tolar \& Hogan, 2007; Lindquist \& Whitehead, 1986; Morgan, Van Haveren \& Pearson, 2002). Based on the previous literature, the results of the present study may not be reflective of the general population of COs. As previously noted, the 
participants were recruited from social media-based, supportive groups for officers. Research conducted by Morgan and colleagues (2002) found that officers identified peer and social support as one mitigating factor of burnout. The population surveyed for this study may be more aware of their professional burnout, and therefore actively seek out and acquire higher rates of peer support, which may, in turn, help to allay burnout symptoms. This may, in part, account for inconsistencies between the present results and previous research regarding levels of $\mathrm{CO}$ burnout.

Preliminary analysis of SSEIT scores suggest that COs perceive themselves to have high emotional intelligence. The range of possible scores for the measure is $33-165$. In the present study, the mean score was $122.50(\mathrm{SD}=14.49$, Range=79-156), suggesting that $\mathrm{COs}$ have high self-reported propensity for appraisal and expression of emotion in self and others, a high ability to regulate self-emotions and the emotions of others, and have high utilization of emotions in problem solving. The scores of COs in this study were comparable to those reported by general university students, university students in emotional intelligence training programs, senior managers, parents of university students, and human service professionals such as social workers (Guastello \& Guastello, 2003; Ogin’ska-Bulik, 2005; Saklofske et al., 2007; Schutte \& Malouff, 2002; Totterdell \& Holman, 2003). The implications of COs' reported levels of emotional intelligence will be further addressed in the discussion of the primary hypotheses of the study.

\section{Discussion of CBI as a Moderator of OMI and SSEIT}

Results of the primary analysis suggest that $\mathrm{CO}$ opinions about mental illness do not significantly predict levels of emotional intelligence. In previous research, no clear relationship between the two constructs has been established. The premise of emotional intelligence is "the ability to monitor one's own and others' feelings and emotions, to discriminate among them and 
to use this information to guide one's thinking and actions" (Salovey \& Mayer, 1990, p. 189). It was hypothesized that having high levels of emotional intelligence would be correlated with having more positive opinions about individuals with mental illness, as the ability to appraise and understand the emotions of others may allow for increased empathy and mental flexibility (Salovey \& Mayer, 1990).

There are several criticisms of EI. Among them is the lack of consensus about an operational definition of the theoretical construct. Many definitions have been generated, and it has been suggested that EI is difficult to differentiate from other constructs such as social competence. From the perspective of this criticism, officers may have an ability to recognize and respond to a typical range of emotions. However, typical emotional responses differ from mental illnesses, which are conditions marked by changes in thinking, behavior, and emotion (American Psychiatric Association, 2018). The literature suggests that EI is an indicator of effective leadership and interpersonal functioning (Goleman, 2002). These abilities may not extend into the realm of changes in perception and opinions about constructs such as mental illness.

Interestingly, results suggest that when accounting for OMI scores, burnout independently predicts emotional intelligence, suggesting that, as levels of burnout increase, levels of emotional intelligence also increase. The original hypothesis was the inverse of these findings and predicted that lower levels of burnout would be associated with higher levels of EI. COs who have higher levels of EI would theoretically have an increased ability to appraise and understand their own emotions (Salovey \& Mayer, 1990). Having an increased awareness of one's emotional state may enable correctional officers to be more cognizant of their levels of burnout. They may be more effective at recognizing the symptoms of burnout including 
psychological exhaustion as it relates to their personal lives, jobs, and inmates (Kristensen, 2005).

If COs with higher levels of EI are better able to recognize burnout as it occurs, there could be potential benefits. Awareness and willingness to communicate about the physical and emotional toll of burnout may increase the likelihood that COs seek supportive service or engage in activities that reduce the symptoms of burnout. Being aware of and expressing burnout opens the door for administrative staff and psychologists to intervene. These officers may be more motivated to speak with their fellow officers about their own experiences, empathize with the experiences of other officers, and participate in developing further dialogue and helpful interventions to support one another. There could be the potential for collaboration among COs and administrators to address the critical problem of burnout in correctional settings.

This finding also potentially speaks to the need for increasing $\mathrm{CO}$ emotional intelligence through related trainings. It is not ideal for COs to experience the increase in burnout that may accompany being more aware of emotions. However, working to increase EI may subsequently create a unique opportunity for supervisory and administrative staff to provide officers with the resources and education they need to effectively manage burnout and related symptoms as they arise, or come into their awareness.

Just as there may be a need for providing COs with training to increase emotional intelligence, it may also be equally as important to address the high levels of burnout that are found in this profession. Correctional facilities would likely benefit from developing strategies to provide burnout education and helpful intervention strategies. This could have several benefits, including those supported in previous literature such as decreased turnover, decreased 
absenteeism, increased productivity, and better health outcomes for officers (Brough \& Williams, 2007; Finn, 2000; Lambert et al., 2007).

Although the relationship between EI and burnout was not found to be significant in the direction that was originally hypothesized, this interesting finding provided valuable insight into the perspectives of COs. The finding still supports the need for increasing EI among officers, if not for decreasing burnout, at least for helping COs become more aware of the experience of burnout.

\section{Discussion of Mental Health Training as a Moderator of OMI and SSEIT}

The results of the study suggest that mental health training does not significantly predict levels of EI. As previously discussed, there appear to be inconsistencies in self-reported hours of mental health training, rendering this variable potentially problematic. It may not be an accurate reflection of the actual hours of mental health training, separate from pre-service training, that COs have completed. However, if officers are accurately reporting their hours, this speaks to systemic problems in the delivery of mental health training. If officers are not being provided with fundamental education on mental illness in correctional facilities, their ability to effectively work with SMI inmates could be significantly diminished.

Despite the concerns with this variable, the results are consistent with what has been found in some previous studies. COs who have completed mental health training feel more equipped to interact with inmates with mental illness than their non-trained counterparts (Gibb, 2015; Parker, 2009; Petracek, 2012). An increased skillset does not necessarily equate to a changed mindset. Petracek's research found that COs who received crisis intervention training (CIT) maintained similar perceptions of offenders with mental illness as their non-trained counterparts, despite an improved perceived ability to work with the population (2012). The 
literature suggests that COs benefit from mental health training as it relates to improving jobrelated skills such as decreased use of force and management of mentally ill offenders. However, the type of training received may account for the lack of change in opinions and perceptions. To change opinions about mental illness, specific training that addresses increasing empathy for individuals with mental illnesses, such as CRT may also be required.

The use of mental health training in other law enforcement fields has shown more promising results related to improvement in perceptions about individuals with mental illness. For example, Compton and colleagues (2006) found that police officers who receive Crisis Response Training (CRT) reported improved attitudes about the aggressiveness of individuals with schizophrenia, increased support for mental health treatment programs, increased support for substance abuse treatment programs, and increased knowledge about schizophrenia. It may be beneficial for correctional facilities to implement training protocols such as CRT, as this is an evidence-based model that has shown promise in improving both attitudes and skill sets.

The present study suggests that officers maintain negative opinions about mental illness while also reporting high levels of emotional intelligence. Results from the analysis also indicate that, when accounting for Mental Health Training scores, OMI scores do not predict EI. The theoretical framework of emotional intelligence has yielded three primary models for understanding emotional intelligence. Bar-on's (2004) model is an ability model, which includes the ability to be aware of, understand, and express emotion, understand and relate to others, manage strong emotion and impulses, and adapt solve interpersonal problems or conflicts. Mayer and Salovey's (1997) model suggests that EI is the ability to perceive emotions and access emotional content. Through this process, emotions can be generated that assist our thought processes, which promotes emotional and intellectual growth. Goleman's (1996) model was 
developed based on workplace performance, and includes self-awareness, self-management, social awareness, and relationship management. Although each theoretical model differs slightly, they each maintain that EI includes the ability to effectively understand emotions and apply this understanding to various life domains and interactions. The application of emotional intelligence is where the models diverge. Previous literature seems to suggest that there are interpersonal benefits of high EI for COs, in the sense that they have the skills necessary to effectively interact with inmates. The intrapersonal aspects of EI do not appear to be well understood in the context of $\mathrm{COs}$

Bar-on's (2004) model of EI is an ability model that includes the ability to understand and relate to others. Based on this theoretical perspective, it would be appropriate to hypothesize that COs with higher levels of emotional intelligence would also have more positive opinions about individuals with mental illness, by virtue of empathy and perspective-taking. This is not, however, supported in the present study. It is possible that the measures used to assess emotional intelligence are better equipped to measure the functional and skill-based aspects of the construct, rather than the perception and opinions-based aspects that are more likely to be associated with more positive attitudes toward different groups, including individuals with mental illness.

An additional criticism of EI pertains to the way that the construct is measured. The validity of various measures of emotional intelligence have been shown to vary greatly based on the populations surveyed. Additionally, it has been argued that EI is merely a facet of constructs such as general intelligence, social conformity, or personality characteristics, rather than an independent construct. This argument suggests that, when measuring EI, researchers may be studying other constructs. 
Given the criticism that measuring EI may be better understood as a survey of personality characteristics, consideration must also be given to personality theory. Gordon Allport (1961) posited that, "Personality is the dynamic organization within the individual of those psychophysical systems that determine his [sic] characteristics, behavior, and thought” (p. 28). Personality theory and assessment have been used in previous research to better understand the personality characteristics of COs. When conducting cluster analyses of CO MMPI personality profiles, officers engage in mildly defensive responding. They tend to portray themselves in a positive light. Additionally, cluster analyses have found that profiles suggest that officers are likely to be outgoing, ambitious, and action oriented. They also tend to be manipulative in social relationships, have difficulty experiencing concern for the feelings and needs of others, and are infrequently thought-oriented (Holland, Heim \& Holt, 1976; Shusman, Inwald \& Landa, 1991). These findings support the literature that suggests that increased EI may be related to skill acquisition, rather than empathic responding. The findings also seem to support the results of the current study. Results suggest that officers maintain negative opinions about inmates with mental illness, which is consistent with difficulty experiencing concern for the feelings and needs of others. However, COs in the study perceive that they have somewhat high levels of emotional intelligence, consistent with skill-related, emotionally intelligent behaviors.

In the current study, mental health training was not found to moderate the relationship between OMI and EI. As previously discussed, it is possible that the study measured perception of skill in interacting with inmates with mental illness, rather than levels of empathy and perspective taking. Because the sample contained COs from various states and federal correctional systems, there is considerable variability in the type of mental health training received. One possible explanation is that there was no way of controlling for the quality of the 
curriculum or the instructor. A trained, qualified, interested, and engaged correctional professional should ideally implement the trainings. Accounting for the delivery of training is a key factor in ensuring that information is disseminated in a meaningful way. The absence of high-quality training provided by a high-quality trainer could account for the lack of significance in the effect of mental health training on the relationship between OMI and EI.

The development and use of evidence-based mental health training for correctional officers should be addressed, so that the training COs receive not only addresses skill-related behaviors, but also aims to improve the attitudes that officers have about this population. Improved attitudes are related to improved cohesion between officers and mental health professionals, aversion of crisis escalation, and decreases in use of force (Applebaum, Hickey, \& Packer, 2001; Davidson, 2004; Packer, 2009; Powers-Margo, 2015). Officer safety is subsequently improved if crises and use of force are mitigated.

\section{Limitations of The Study}

\section{Threats to Internal Validity}

Within the study, there are potential threats to internal and external validity that may have affected the results. Subject characteristics are one consideration. In the study, a national sample was acquired through recruitment on closed, social media-based, supportive groups for COs. The aim of this recruitment method was to collect a broad and diverse sample for generalizability of the results. As COs who participated in the study were recruited from supportive groups, the sample may be representative of officers who seek out support from their peers and have a strong sense of professional identity and connection. Their current support structures may serve to improve some of the factors the study attempted to measure such as their levels of burnout and levels of emotional intelligence. 
Data collector characteristics may have also affected the results of the study. As discussed in the literature review, mental health professionals and COs are often at odds in correctional environments due to the differences in their roles and professional responsibilities. The researcher was identified as a student completing a dissertation project in the field of psychology. Research suggests that COs perceive that psychologists and mental health professionals shelter offenders from receiving consequences for negative behaviors (Appelbaum, et al., 2001). Knowing that the researcher was associated with the field of psychology, participants' willingness to be forthcoming in their responses regarding perceptions of mental illness, levels of burnout, and personal EI may have been affected.

Instrumentation may also have impacted the results of the study. When selecting scales for this study, all measures were chosen based on their adequate psychometric properties and prior use with COs or human service professionals. In addition to these important components, they were also chosen for their accessibility and cost effectiveness. All measures were selfreport, which can be accompanied by a number of limitations such as social desirability bias or exaggeration. The measures selected for the study also lack validity scales. It is not possible to detect if participants responded in a forthright manner if measures do not contain these indicators. This is a significant limitation of the study.

\section{Threats to External Validity}

One concern of the study was the mental health training variable and the accuracy of the figures reported. The method of sampling could have also played a role in the problematic nature of this variable. To decrease the variation seen in the mental health training variable, it may have been beneficial to collect data from officers at one identified institution, rather than collecting a national sample. Recruitment from one location would help to refine what was considered mental 
health training and identify the exact type of training received. Additionally, uniform training would likely be offered or mandated to all officers at an individual correctional facility. Ensuring that the sample had received similar training may have increased the reliability of the variable. However, limiting the study in this way would inevitably detract from the generalizability to larger CO populations, threatening external validity.

For this study, electronic means of data collection were used to survey participants. There are several drawbacks to using this method of data collection. Poor response rate is often a consequence of online survey administration. Ways to combat low response rates include sending frequent follow-up requests for participation and providing monetary incentives for participation (Coughlan, Cronin, \& Ryan, 2009). Both suggestions were used in the study. Despite implementing suggestions found in the research, a small, but adequate sample size was obtained. A larger sample size would have increased power and subsequently increased the generalizability of the results to the larger $\mathrm{CO}$ population.

Another emerging drawback of internet-based data collection is the potential of fraudulent participation in studies to acquire compensation. This can pose significant concerns to the validity of the study. Over the past decade, the use of incentives for research has become more popular as a result of a decrease in research study participation. This presents a unique opportunity for "fraudsters" to create fake IP addresses, complete surveys, and receive incentives. This was a problem that was encountered, addressed, and remedied during the present study. There are several types of internet-based fraud that can occur. According to Teitcher and colleagues (2015), the three primary problems are as follows: "1) eligible individuals who take a study twice, presumably without malicious intent; 2) eligible individuals who take a study 
repeatedly to receive additional compensation; and 3) ineligible individuals who take a study once or repeatedly to profit from compensation" (p. 116).

The current study remedied these problems through using recommendations outlined by Teitcher and colleagues (2015) to minimize the likelihood of fraudulent data, including checking for consistencies, adding a captcha verification, providing compensation information at the end of the survey, checking for duplicate IP addresses, adding language that states that fraudulent surveys will not be compensated, not compensating all participants, and paying less money to participants. Despite taking several measures to protect the data from "fraudsters," there is no way to ensure that the entire data set is legitimate and creates a potential threat to the validity of the study.

\section{Strengths of the Study}

Despite lacking statistically significant findings, the study is relevant in the current climate in the United States and our criminal justice system. The number of individuals with mental illness who are housed in correctional settings is high. This has led to several challenges related to managing and providing mental health services to the population. On the front lines of managing this challenge are COs. $\mathrm{CO}$ experiences and perceived competence for working with this population should be considered. If nothing is done to reduce the large number of SMI offenders found in correctional settings, COs should be provided with the knowledge and resources to adequately understand and intervene when mental health crises emerge. The study aimed to better understand $\mathrm{CO}$ perceptions of mental illness and factors that may influence their ability to effectively interact with SMI inmates, including EI, burnout, and mental health training. The intent was to contribute to the existing literature and to provide possible direction for improving existing mental health training models, such as the incorporation of EI training. 
The primary strength of the study is that it is grounded in peer reviewed literature and psychological theory. Using research and theory as a foundation provided a clear structure and sound research questions and hypotheses. Although the six primary hypotheses of the study were not found to be statistically significant, preliminary analyses suggest that COs endorsed burnout and negative opinions about mental illness. This is consistent with previous literature and serves to confirm that more work should be done to provide COs with the knowledge and resources to mitigate burnout and improve opinions about a population with which they work closely.

\section{Implications for Counseling Psychology}

In recent years, counseling psychologists have found themselves working in nontraditional settings with diverse clientele. The values of counseling psychology, including focuses on strength-based approaches, developmental approaches and culturally competent treatment, make working in correctional settings a unique and good fit for counseling psychologists. In corrections, the work of counseling psychologists is varied. In addition to providing clinical services to the inmate population, psychologists are tasked with working as effective members of administrative and correctional interdisciplinary teams as well as providing education and training to staff regarding psychological concepts.

Identifying CO opinions about offenders with mental illness and better understanding the relationships among those opinions and contextual factors such as burnout and emotional intelligence could assist psychologists with understanding the dynamic interplay between officers and the inmates they encounter daily. Counseling psychologists often rely on COs to identify acute changes in inmates with mental illness. Developing an understanding of, and empathy for the $\mathrm{CO}$ experience could assist in forging stronger relationships with correctional staff. Additionally, understanding the relationships among these constructs could assist 
psychologists and facility administrators in working effectively as part of interdisciplinary teams to determine appropriate interventions and mental health training modalities for COs. Counseling psychologists have a unique opportunity to apply their knowledge and skills in correctional settings to make service delivery and system level changes that benefit both COs and inmates

\section{Future Research and Conclusion}

This study aimed to expand the current literature around $\mathrm{CO}$ opinions about inmates with mental illness, through determining if relationships exist among opinions, burnout, and EI. Additionally, the study attempted to better understand the role of mental health training in levels of emotional intelligence and opinions about mental illness. The purpose of the study was to assist mental health professionals and administrative personnel in better understanding the unique perceptions COs maintain about inmates with mental illness. A better understanding of these relationships could assist psychologists when working as part of interdisciplinary teams alongside COs. Additionally, this knowledge could assist in the development of mental health training for correctional officers. The results of the study were found to be insignificant, indicating that no relationships among the identified variables exist.

Future studies may benefit from a larger sample size to increase power. The total sample size was 105 . To estimate the sample size for the study, an a priori power analysis was calculated using $\mathrm{G}^{*}$ Power software (Faul, et al., 2009). Based on an alpha $(\alpha)$ level of .05, the power analysis calculated a minimum estimation of 77 participants to obtain statistical power of .80 and a medium effect size. The results of the study were meant to be generalized to the larger United States CO population. Although the study surpassed the minimum estimation of participants, a larger sample would have been beneficial in increasing the accuracy and reliability of results by providing more precise mean values and identifying outliers in the data. 
Future researchers should consider several areas on which to focus their studies. It may be beneficial to identify or develop training for COs that address skill acquisition as well as interventions aimed at improving perceptions of offenders with serious mental illness. Several lines of inquiry have been established in other law enforcement fields. Implementation and evaluation of such programs in correctional settings may provide new avenues of training for COs.

A pertinent concern that was identified in the study is $\mathrm{CO}$ burnout. Officers experience high levels of burnout and should be provided with education and resources to identify and combat burnout, as decreasing burnout has benefits for both correctional institutions and officers. Future research should aim to better understand $\mathrm{CO}$ burnout and identify practical, effective means of mitigating symptoms in this population.

Future researchers may also benefit from conducting research at a targeted, limited number of correctional institutions to account for variation in factors such as hours and type of mental health training. This would also likely ensure that a homogenous group of participants is not identified, such as was found in this study. As officers were recruited from support groups, they may differ in experiences and perceptions from the general $\mathrm{CO}$ population.

There are several theoretical considerations to account for when studying emotional intelligence. Future research should attempt to determine if relationships between opinions about mental illness and EI exist. Additionally, operationalizing the definition of EI in correctional settings may be beneficial when conducting future research. Qualitative studies or scales that assess the traits identified in the operational definition should be used. It may be beneficial to focus efforts on talking to COs to better understand their experiences with SMI inmates, the trainings they have completed, and what training they believe would be beneficial in assisting 
them in working with this population. If future researchers utilize self-report measures, they should consider the number of associated limitations. Using instruments with validity indices may increase the likelihood that researchers are acquiring results that are an accurate reflection of participants' experiences. 


\section{REFERENCES}

Abraham, R. (2000). The role of job control as a moderator of emotional dissonance and emotional-intelligence-outcome relationships. The Journal of Psychology, 134 (1), 169184. doi: 10.1080/00223980009600860

Adams-Flores, N., Catlett, T., Montoya, E., Richman, M., Toche, D., \& Washington, T. (2017). Behavioral health resource guide by the Correctional Behavioral Health Working Group, Coalition of Correctional Health Authorities, and American Correctional Association $[\mathrm{PDF}]$. Retrieved from http://www.aca.org/ACA_Prod_IMIS/DOCS/OCHC/CCHA\%20Behavioral\%20Health\% 20Resource\%20Guide.pdf

Adams K, \& Ferrandino J. (2008). Managing mentally ill inmates in orisons. Criminal Justice and Behavior. 35(9),13-927. doi: 10.1177/0093854808318624

Al Ali, O. E., Garner, I., \& Magadley, W. (2012). An exploration of the relationship between emotional intelligence and job performance in police organizations. Journal of Police and Criminal Psychology, 27(1), 1-8. doi:10.1007/s11896-011-9088-9

Allport, G. W. (1937). Personality: A psychological interpretation. New York: H. Holt and. Company.

American Correctional Association. (2017). Public Correctional Policies [PDF]. Retrieved from http://www.aca.org/ACA_Prod_IMIS/docs/GovernmentAffairs/ACA_PUBLIC_CORRE CTIONAL_POLICIES_BOOK.pdf?WebsiteKey=139f6b09-e150-4c56-9c66-

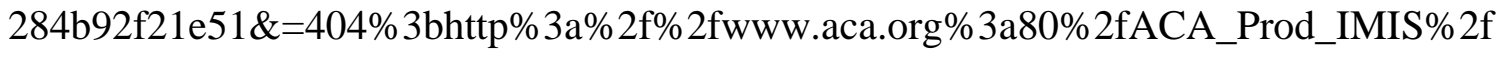
ACA_Member\%2fdocs\%2fGovernmentAffairs\%2fACA_PUBLIC_CORRECTIONAL_ POLICIES_BOOK.pdf 
American Psychiatric Association. (2018). What is mental illness? [Webpage] Retrieved from https://www.psychiatry.org/patients-families/what-is-mental-illness

American Psychological Association. (2017). Position statement on psychiatric services in jails and prisons [PDF]. Retrieved from file://C:/Users/HC8191/Downloads/Position-2007Jails-Prisons.pdf

Anasseril, D. (2007). Care of the mentally ill in prisons: Challenges and solutions. Journal of the American Academy of Psychiatry and the Law Online, 35(4) 406-410. doi: $10.1177 / 2158244013497029$

Anderson, C. (2010). Presenting and evaluating qualitative research. American Journal of Pharmaceutical Education, 74(8), 141. doi: 10.5688/aj7408141

Anfang, S. A., \& Applebaum, P. S. (2006). Civil commitment- the American experience. The Israel Journal of Psychiatry and Related Sciences, 43(3), 209-218. doi: $10.3109 / 10398562.2010 .507816$

Appelbaum, P. S. (1990). Washington v. Harper: Prisoners' rights to refuse antipsychotic medication. Psychiatric Services, 41(7), 731-732. doi: 10.1176/ps.41.7.731

Appelbaum, K. L., Hickey, J. M., \& Packer, I. (2001). The role of correctional officers in multidisciplinary mental health care in prisons. Psychiatric Services, 52(10), 1343-1347. doi: 10.1176/appi.ps.52.10.1343

Aron, A., Aron, E., \& Coups, E. J. (2014). Statistics for psychology (6th ed.). Harlow: Pearson. doi: 10.1965367/138861_14

Aufderheide, D. (2014). Mental illness in America's jails and prisons: Toward a public safety/public health model [Website]. Retrieved from 
http://healthaffairs.org/blog/2014/04/01/mental-illness-in-americas-jails-and-prisonstoward-a-public-safetypublic-health-model/

Austin, E. J., Saklofske, D. H., Huang, S. H. S., \& McKenney, D. (2004). Measurement of trait emotional intelligence: Testing and cross-validating a modified version of Schutte et al.'s (1998) measure. Personality and Individual Differences, 36(1), 555-562. doi: $10.1016 / \mathrm{s} 0191-8869(03) 00114-4$

Baillargeon, J., Binswanger, I. A., Penn, J. V., Williams, B. A., \& Murray, O. J. (2009). Psychiatric disorders and repeat incarcerations: The revolving prison door. American Journal of Psychiatry, 166(1), 103-109. doi: 10.1176/appi.ajp.2008.08030416

Barlow, D. H. (Ed.). (1985). Clinical handbook of psychological disorders: A step-by-step treatment manual. New York, NY: Guilford Press

Bar-On, R. (1997). Bar-On Emotional Quotient Inventory (EQ-i): A test of emotional intelligence. Toronto, Canada: Multi-Health Systems. doi: 10.1037/t04985-000

Bar-On, R. (2004). The Bar-On Emotional Quotient Inventory (EQ-i): Rationale, description and summary of psychometric properties. In G. Geher (Ed.), Measuring emotional intelligence: Common ground and controversy (pp. 115-145). Hauppauge, NY, US: Nova Science Publishers. doi: 10.1007/978-3-319-32637-5_4

Beck, A. J., \& Maruschak, L. M. (2001). Mental health treatment in state prisons, 2000. Washington, DC: US Department of Justice, Office of Justice Programs, Bureau of Justice Statistics [Webpage]. Retrieved from https://www.prisonpolicy.org/scans/bjs/mhtsp00.pdf

Behnke, S. (2001). A question of values. Monitor on Psychology, 32(9), 1-2. doi: 10.1037/e303952004-057 
Boothby, J. L., \& Clements, C. B. (2000). A national survey of correctional psychologists. Criminal Justice and Behavior, 27(6), 716-732. doi: 10.1177/0093854800027006003

Borman, W. C., Penner, L. A., Allen, T. D., \& Motowidlo, S. J. (2001). Personality predictors of citizenship performance. International Journal of Selection and Assessment, 9(1-2), 5269. doi: $10.1111 / 1468-2389.00163$

Borritz, M., Rugulies, R., Christensen, K. B., Villadsen, E., \& Kristensen, T. S. (2006). Burnout as a predictor of self-reported sickness absence among human service workers: prospective findings from three year follow up of the PUMA study. Occupational and Environmental Medicine, 63(2), 98-106. doi: 10.1097/jom.0b013e3181f12f95

Boyatzis, R. E., Cowan, S. S., \& Kolb, D. A. (1995). Innovations in professional education: Steps on a journey to learning. San Francisco, CA: Jossey-Bass.

Boyatzis, R. E., Stubbs, E. C., \& Taylor, S. N. (2002). Learning cognitive and emotional intelligence competencies through graduate management education. Academy of Management Learning \& Education, 1(2), 150-162. doi:10.5465/amle.2002.8509345

Brackett, M. A., Mayer, J. D., \& Warner, R. M. (2004). Emotional intelligence and its relation to everyday behavior. Personality and Individual Differences, 36, 1387-1402. doi: $10.1016 / \mathrm{s} 0191-8869(03) 00236-8$

Brackett, M. A., Rivers, S. E., \& Salovey, P. (2011). Emotional intelligence: Implications for personal, social, academic, and workplace success. Social and Personality Psychology Compass, 5(1), 88-103. doi: 10.1111/j.1751-9004.2010.00334.x

Brackett, M. A., Warner, R. M., \& Bosco, J. S. (2005). Emotional intelligence and relationship quality among couples. Personal Relationships, 12(1) 197-212. doi: 10.1111/j.1350-4126.2005.00111.x 
Bronstein, L. R. (2003). A model for interdisciplinary collaboration. Social work, 48(3), 297306. doi: 10.1093/sw/48.3.297

Brough, P., \& Williams, J. (2007). Managing occupational stress in a high-risk industry:

Measuring the job demands of correctional officers. Criminal Justice and Behavior, 34(4), 555-567. doi: 10.1177/0093854806294147

Brunetto, Y., Teo, S. T., Shacklock, K., \& Farr-Wharton, R. (2012). Emotional intelligence, job satisfaction, well-being and engagement: explaining organizational commitment and turnover intentions in policing. Human Resource Management Journal, 22(4), 428-441. doi: 10.1111/j.1748-8583.2012.00198.x

Bureau of Justice Statistics. (2016). Prisoners in 2016 [Webpage]. Retrieved from https://www.bjs.gov/content/pub/pdf/p16_sum.pdf

Bureau of Labor Statistics. (2017). U.S. Department of Labor, occupational outlook handbook, correctional officers and bailiffs [Webpage]. Retrieved from https://www.bls.gov/ooh/protective-service/correctional-officers.htm

Burnham, J. C. (2006). A clinical alternative to the public health approach to mental illness: A forgotten social experiment. Perspectives in Biology and Medicine, 49(2), 220-237. doi: 10.1353/pbm.2006.0021

Callahan, L. (2004). Correctional officer attitudes toward inmates with mental disorders. International Journal of Forensic Mental Health, 3(1), 37-54. doi: $10.1080 / 14999013.2004 .10471195$

Campbell, C., Joiner, B. L., Ferber, R., Sheatsley, P., Turner, A., \& Waksberg, J. (1981). What Is a Survey? The American Statistician, 35(3), 159. doi:10.2307/2683989 
Carlson, J. R., Anson, R. H., \& Thomas, G. (2003). Correctional officer burnout and stress: Does gender matter?. The Prison Journal, 83(3), 277-288. doi: 10.1177/0032885503256327

Center, T. A. (2014). How Many individuals with serious mental illness are in jails and prisons?. Arlington: Treatment Advocacy Center [Webpage]. Retrieved from https://www.treatmentadvocacycenter.org/evidence-and-research/learn-more-about/3695

Cherniss, C. (2010). Emotional intelligence: Toward clarification of a concept. Industrial and Organizational Psychology, 3, 110-126. doi: 10.1111/j.1754-9434.2010.01231.x

Ciarrochi, J., Chan, A.Y.C., \& Bajgar, J. (2001). Measuring emotional intelligence in adolescents. Personality and Individual Differences, 31(1), 1105-1119. doi: $10.1016 / \mathrm{S} 0191-8869(00) 00207-5$

Cieslak, R., Korczynska, J., Strelau, J., \& Kaczmarek, M. (2008). Burnout predictors among prison officers: The moderating effect of temperamental endurance. Personality and Individual differences, 45(7), 666-672. doi: 10.1016/j.paid.2008.07.012

Cloyes, K. G. (2007). Challenges in residential treatment for prisoners with mental illness: A follow-up report. Archives of Psychiatric Nursing, 21(4), 192-200. doi: 10.1016/j.apnu.2007.02.009

Cohen, J., \& Struening, E. L. (1962). Opinions about mental illness in the personnel of two large mental hospitals. The Journal of Abnormal and Social Psychology, 64(5), 349. doi: $10.1037 / \mathrm{h} 0045526$

Compton, M., Esterberg, M., McGee, R., Kotwicki, R., \& Oliva, J. (2006). Brief reports: Crisis intervention team training: Changes in knowledge, attitudes, and stigma related to schizophrenia. Psychiatric Services, 57(8), 1199-1202. doi 10.1176/appi.ps.57.8.1199 
Connery, H., \& Davidson, K. (2006). A survey of attitudes to depression in the general public: A comparison of age and gender differences. Journal of Mental Health, 15, 179-189. doi: 10.1080/09638230600608818.

Correctional Officer Education. (2018). Corrections Officer Training [Webpage]. Retrieved from https://www.correctionalofficeredu.org/training/

Correctional Officer.org. (2016). Correctional officer duties [Webpage]. Retrieved from http://www.correctionalofficer.org/faq/correctional-officer-job-description

Cullen, F. T., Link, B., Wolfe, N., \& Frank, J. (1985). The social dimensions of correctional officer stress. Justice Quarterly, 2(4), 505-533. doi: 10.1080/07418828500088711

David, S. A. (2005). Emotional intelligence: Developmental antecedents, psychological and social outcomes (Doctoral dissertation). University of Melbourne: Melbourne, Australia.

Davidson, M. L. (2014). A Criminal Justice System-Wide Response to Mental Illness. Criminal Justice Policy Review, 27(1), 46-75. doi:10.1177/0887403414554997

Davidson, R. J., Jackson, D. C., \& Kalin, N. H. (2000). Emotion, plasticity, context, and regulation: Perspectives from affective neuroscience. Psychological Bulletin, 126(6), 890. doi: 10.1037//0033-2909.126.6.890

Denhof, M. D., Spinaris, C. G., \& Morton, G. R. (2014). Occupational stressors in corrections organizations: Types, effects and solutions. US Department of Justice, National Institute of Corrections [Webpage]. Retrieved from https://info.nicic.gov/nicrp/system/files/028299.pdf

Deutsch, A. (1948). The shame of the states (1st ed). New York, NY: Harcourt Brace. Dillman, D. A. (1978). Mail and telephone surveys: The total design method. New York, NY: Wiley-Interscience. doi: 10.2307/2068433 
Downey, L. A., Papageorgiou, V., \& Stough, C. (2006). Examining the relationship between leadership, emotional intelligence and intuition in senior female managers. Leadership \& Organization Development Journal, 27(4), 250-264. doi:10.1108/01437730610666019

Drolen, C. S. (1993). The effect of educational setting on student opinions of mental illness. Community Mental Health Journal, 29(3), 223-234. doi: 10.1007/bf00778808

Dvoskin, J. A., \& Spiers, E. M. (2004). On the role of correctional officers in prison mental health. Psychiatric Quarterly, 75(1), 41-59. doi: 10.1023/b:psaq.0000007560.09475.a0

Eack, S. M., Hogarty, G. E., Greenwald, D. P., Hogarty, S. S., \& Keshvan, M. S. (2007). Cognitive enhancement therapy improves emotional intelligence in early course schizophrenia: Preliminary effects. Schizophrenia Research, 89(1), 308-311. doi: 10.1016/j.schres.2006.08.018

Emmerling, R. J., \& Goleman, D. (2003). Emotional intelligence: Issues and common misunderstandings. Issues and Recent Developments in Emotional Intelligence, 1(1), 132. doi: 10.1037/e576082010-001

Faul, F., Erdfelder, E., Buchner, A., \& Lang, A-G. (2009). Statistical power analyses using G*Power 3.1: Tests for correlation and regression analyses. Behavior Research Methods, 41(4), 1149-1160. doi: 10.3758/BRM.41.4.1149.

Federal Bureau of Prisons (2020). Staff Gender [Webpage]. Retrieved from https://www.bop.gov/about/statistics/statistics_staff_gender.jsp

Feldman, S. (2003). Reflections on the 40th anniversary of the US community mental health centers act. Australian \& New Zealand Journal of Psychiatry, 37(6), 662-667. doi: 10.1080/j.1440-1614.2003.01268.x 
Fellner, J. (2006). A corrections quandary: Mental illness and prison rules. Harvard Civil RightsCivil Liberties Law Review,41, 391-412.

Finn, P. (2000). Addressing correctional officer stress: Programs and strategies. Washington, DC: US Department of Justice, Office of Justice Programs, National Institute of Justice.

Fuller, D.A., Sinclair, E., Geller, J., Quanbeck, C., Snook, J. (2016). Going, going, gone: Trends and consequences of eliminating state psychiatric beds, 2016. Arlington, VA: Treatment Advocacy Center [Webpage]. Retrieved from https://www.treatmentadvocacycenter.org/storage/documents/going-going-gone.pdf

Galanek, J. (2015). Correctional officers and the incarcerated mentally ill: Responses to psychiatric illness in prison. Medical Anthropological Quarterly, 29(1), 116-136. doi: 10.1111/maq.12137

George, J. M. (2000). Emotions and leadership: The role of emotional intelligence. Human Relations, 53, 1027-1055. doi: 10.1177/0018726700538001

Geller, A., \& Curtis, M. A. (2011). A sort of homecoming: Incarceration and the housing security of urban men. Social Science Research 40(1), 1196-1213. doi: 10.1016/j.ssresearch.2011.03.008

Gibb, B. (2015). A new skill for corrections professionals: Mental health first aid [Webpage]. Retrieved from http://www.corrections.com/news/article/40837-a-new-skill-forcorrections-professionals-mental-health-first-aid

Gibson, G. (2018). In sight: Women of corrections on their paths, their jobs and advice [Website]. Retrieved from http://www.aca.org/ACA_Prod_IMIS/DOCS/Corrections\%20Today/2018\%20Articles/Ja nuary\%202018/Gibson.pdf 
Gillepie, K. (2015). Training helps Wash. officers deal with mental illness at jail [Webpage]. Retrieved from https://www.correctionsone.com/correctionstraining/articles/8353712-Training-helps-Wash-officers-deal-with-mental-illness-at-jail/

Glaze, L., \& Herberman, E. (2012). Correctional populations in the United States, 2012 [Webpage]. Retrieved from http://www.bjs.gov/index.cfm?ty=pbdetail\&iid=4843

Goleman, D. (1996). Emotional intelligence. Why it can matter more than IQ. Learning, 24(6), 49-50.

Goleman, D. (2006). Social Intelligence: The New Science of Human Relationships. New York, NY: Bantam. doi: 10.1002/jpoc.20099

Guastello, D. D., \& Guastello, S. J. (2003). Androgyny, gender role behaviour, and emotional intelligence among college students and their parents. Sex Roles, 49, 663-673. doi: doi:10.1023/b:sers.0000003136.67714.04

Harmon-Nary, J. (2013). Correctional officer perceptions: Opinions on mental illness, mental health treatment and officer training (Order No. 3536313). Available from ProQuest Dissertations \& Theses Global. (1312510895). Retrieved from http://www.libproxy.wvu.edu/login?url=https://search-proquestcom.www.libproxy.wvu.edu/docview/1312510895?accountid=2837

Hasin, D. (1988). Age and recognition of depression: Implications for a cohort effect in major depression. Psychological Medicine, 18, 683-688. doi:10.1017/s0033291700008369

Hewer, M. (2016). Intelligence over time [Webpage]. Retrieved from https://www.psychologicalscience.org/observer/intelligence-over-time

Hills, H., Sigfried, C., \& Ickowitz, A. (2004). Effective prison mental health services: Guidelines to expand and improve treatment. Washington, DC: US Department of Justice: National 
Institute of Corrections [Webpage]. Retrieved from https://info.nicic.gov/nicrp/system/files/018604.pdf

Hoigaard, R., Giske, R., \& Sundsli, K. (2012). Newly qualified teachers’ work engagement and teacher efficacy influences on job satisfaction, burnout, and the intention to quit. European Journal of Teacher Education, 35(3), 347-357. doi:

$10.1080 / 02619768.2011 .633993$

Holland, T. R., Heim, R. B., \& Holt, N. (1976). Personality patterns among correctional officer applicants. Journal of Clinical Psychology, 32(4), 786-791. doi: 10.1002/1097-4679

Jonker, C. S., \& Vosloo, C. (2008). The psychometric properties of the Schutte emotional intelligence scale. SA Journal of Industrial Psychology, 34(2), 21-30. doi: 10.4102/sajip.v34i2.689

Kaeble, D., Glaze, L., Tsoutis, A., Minton, T. (2016). Correctional populations in the United States, 2014. Bureau of Justice Statistics [Webpage]. Retrieved from https://www.bjs.gov/content/pub/pdf/cpus14.pdf

Kazantzis, N., Wakefield, A., Deane, F. P., Ronan, K. R., \& Johnson, M. (2009). Public attitudes toward people with mental illness in New Zealand, 1995-1996. The Australian Journal of Rehabilitation Counselling, 15(2), 74-91. doi: 10.1375/jrc.15.2.74

Keeler, C. (2018). Correctional officers' and psychologists' personality traits and perceptions of mentally ill inmates (Order No. 10750262). Available from ProQuest Dissertations \& Theses Global. (2029082590). Retrieved from http://www.libproxy.wvu.edu/login?url=https://search-proquestcom.www.libproxy.wvu.edu/docview/2029082590?accountid=2837 
Kiessling, J. J., \& Andrews, D. A. (1980). Behavior analysis systems in corrections: A new approach to the synthesis of correctional theory, practice, management and research. Canadian Journal of Criminology, 22(1), 412. doi: 10.1177/000486587500800409

Kjerulf, A. (2014). Happy hour is 9 to 5: How to love your job, love your life, and kick butt at work. Copenhagen, Denmark: Pine Tribe. doi: 10.978-0991260911

Kliewer, S. P., Melissa, M., \& Trippany, R. L. (2009). Deinstitutionalization: Its impact on community mental health centers and the seriously mentally ill. Alabama Counseling Association Journal, 35(1), 40-45. doi: 10.id=EJ875402

Kristensen, T. S., Borritz, M., Villadsen, E., \& Christensen, K. B. (2005). The Copenhagen Burnout Inventory: A new tool for the assessment of burnout. Work \& Stress, 19(3), 192207. doi: $10.1080 / 02678370500297720$

Kroes, W. (1986). Society's victim: The police officer. Springfield, IL: Charles C. Thomas.

Kropp, P. R., Cox, D., Roesch, R., \& Eaves, D. (1989). The perceptions of correctional officers toward mentally disordered offenders. International Journal of Law and Psychiatry, 12(1), 181-188. doi: 10.1016/0160-2527(89)90006-х

Lamb, H. R., \& Weinberger, L. E. (2005). The shift of psychiatric inpatient care from hospitals to jails and prisons. Journal of the American Academy of Psychiatry and the law Online, 33(4), 529-534. doi: 10.1176/ps.49.4.483

Lambert, E. G., Cluse-Tolar, T., \& Hogan, N. L. (2007). This job is killing me: The impact of job characteristics on correctional staff job stress. Applied Psychology in Criminal Justice, 3(2). doi: 10.4324/9780203832998

Liebling, A., Price, D., \& Shefer, G. (2010). The prison officer. New York, NY: Willan Publishing. 
Liebling, A., Price, D., \& Shefer, G. (2011). The prison officer (2nd ed.). New York, NY: Willan Publishing. doi: 10.281699882

Lopes, P. N., Brackett, M. A., Nezlek, J. B., Schutz, A., Sellin, I., \& Salovey, P. (2004). Emotional intelligence and social interaction. Personality and Social Psychology Bulletin, 30, 1018-1034. doi: 10.1177/0146167204264762

Lopes, P. N., Salovey, P., Côté, S., \& Beers, M. (2005). Emotion regulation ability and the quality of social interaction. Emotion, 5(1), 113-118. doi: 10.1037/1528-3542.5.1.113

Ma, C. C., Andrew, M. E., Fekedulegn, D., Gu, J. K., Hartley, T. A., Charles, L. E., ... \& Burchfiel, C. M. (2015). Shift work and occupational stress in police officers. Safety and Health at Work, 6(1), 25-29. doi: 10.1016/j.shaw.2014.10.001

Manzella, L., \& West, B. (2003). Emotional intelligence and how it contributes to officer safety. Corrections Today, 65(3), 84. doi: 10.10286306

Mauro, M. (2009). My work as a psychologist in prison [Webpage]. Retrieved from https://www.psychologytoday.com/blog/take-all-prisoners/200904/my-workpsychologist-in-prison

Marrow, C. C., Jarrett, M. Q., \& Rupinski, M. T. (1997). An investigation of the effect and economic utility of corporate-wide training. Personnel Psychology, 29, 337- 343. doi: 10.1111/j.1744-6570.1997.tb00902.x

Martelli, T., Waters, L., \& Martelli, J. (1989). The police stress survey: Reliability and relation to job satisfaction and organizational commitment. Psychological Reports, 64, 267-73. doi: 10.2466/pr0.1989.64.1.267 
Mayer, J. D., Roberts, R. D., \& Barsade, S. G. (2008). Human abilities: emotional intelligence. Annual Review of Psychology, 59, 507-536. doi: 10.1146/annurev.psych.59.103006.093646

Mayer, J. D., \& Salovey, P. (1997). What is emotional intelligence? Emotional development and emotional intelligence: Implications for educators. New York, NY: Basic Books. doi: $10.1016 / 0160-2896(93) 90010-3$

Mayer, J. D., Salovey, P., Caruso, D. R., \& Sitarenios, G. (2003). Measuring emotional intelligence with the MSCEIT V2.0. Emotion, 3(1), 97-105. doi: 10.1037/15283542.3.1.97

Mayer, J. D., \& Geher, G. (1996). Emotional intelligence and the identification of emotion. Intelligence, 22(2), 89-113. doi: 10.1016/s0160-2896(96)90011-2

Mayer, J. D., \& Mitchell, D. C. (1998). Intelligence as a subsystem of personality: From Spearman's g to contemporary models of hot processing. Advances in Cognition and Educational Practice, 5, 43-75. doi: 10.2190/ic.30.2.g

Mayer, J. D., Salovey, P., \& Caruso, D. R. (2004). Target articles: Emotional intelligence: Theory, findings, and implications. Psychological Inquiry, 15(3), 197-215. doi: 10.1207/s15327965pli1503_02

McCrae, R. R., \& Costa, P. T., Jr. (1991). Toward a new generation of personality theories: Theoretical contexts for the five-factor model. In J. S. Wiggins (Ed.), The five-factor model of personality: Theoretical perspectives (pp. 51-87). New York, NY: Guilford.

Misis, M., Kim, B., Cheeseman, K., Hogan, N. L., \& Lambert, E. G. (2013). The impact of correctional officer perceptions of inmates on job stress. Sage Open, 3(2), 1-13. doi: 2158244013489695 
Morgan, R. D., Van Haveren, R. A., \& Pearson, C. A. (2002). Correctional officer burnout. Criminal Justice and Behavior, 29(2), 144-160. doi:10.1177/0093854802029002002

MTC Institute. (2017). Women professionals in corrections: A growing asset [Webpage]. Retrieved from https://www.mtctrains.com/wp-content/uploads/2017/06/WomenProfessionals-in-Corrections-A-Growing-Asset.pdf

National Alliance on Mental Illness. (2015). Jailing people with mental illness [Webpage]. Retrieved from http://www.nami.org/Learn-More/Public-Policy/Jailing-People-withMental-Illness

National Alliance on Mental Illness. (2017). Types of mental health professionals [Webpage]. Retrieved from https://www.nami.org/learn-more/treatment/types-of-mental-healthprofessionals

National Commission on Correctional Health Care. (2015). Standards for mental health services in correctional facilities [Webpage]. Retrieved from https://www.ncchc.org/standards

National Council for Behavioral Health. (2015). Community mental health act [Webpage]. Retrieved from https://www.thenationalcouncil.org/about/national-mental-healthassociation/overview/community-mental-health-act/

National Institute of Health. (1993). Fed. Reg.Vol. 58, No. 96. 29422 [Webpage]. Retrieved from http://www.samhsa.gov/sites/default/files/federal-register-notice-58-96-definitions.pdf

National Institute of Health. (2017). Mental Illness [Webpage]. Retrieved from https://www.nimh.nih.gov/health/statistics/mental-illness.shtml

National Institute of Justice. (2014). Recidivism [Webpage]. Retrieved from https://www.nij.gov/topics/corrections/recidivism/pages/welcome.aspx 
Nelis, D., Quoidbach, J., Mokilajczak, M., \& Hansenne, M. (2009). Increasing emotional intelligence: (How) is it possible?. Personality and Individual Differences, 47(1), 36-41. doi: 10.1016/j.paid.2009.01.046

Nelis, D., Kotsou, I., Quoidbach, J., Hansenne, M., Weytens, F., Dupuis, P., \& Mikolajczak, M. (2011). Increasing emotional competence improves psychological and physical wellbeing, social relationships, and employability. Emotion, 11(2), 354. doi:10.1037/a0021554

Nelson, D. B., Low, G. R., \& Ellis, R. (2007). Emotional intelligence: A transformative theory and applied model of positive personal change. Annals of the American Psychotherapy Association, 10(4). doi: 10.12691/education-5-2-15.

Newman, M. A., Guy, M. E., \& Mastracci, S. H. (2009). Beyond cognition: Affective leadership and emotional labor. Public Administration Review, 69(1), 6-20. doi: 10.1111/j.15406210.2008.01935.x

Nowicki, S., \& Duke, M. P. (1994). Individual differences in the nonverbal communication of affect: The Diagnostic Analysis of Nonverbal Accuracy Scale. Journal of Nonverbal Behavior, 18, 9-35. doi: 10.1007/bf02169077

Novella, E. J. (2010). Mental health care and the politics of inclusion: A social systems account of psychiatric deinstitutionalization. Theoretical Medicine and Bioethics, 31(6), 411-427. doi:10.1007/s11017-010-9155-8

Ogińska-Bulik, N. (2005). Emotional intelligence in the workplace: Exploring its effects on occupational stress and health outcomes in human service workers. International Journal of Occupational Medicine \& Environmental Health, 18(2), 167-175. doi: 10.2478/v10001-006-0016-7 
Parker, G. (2009). Impact of a mental health training course for correctional officers on a special housing unit. Psychiatric Services, 60(5), 640-645. doi:10.1176/appi.ps.60.5.640

Pekrun, R., \& Frese, M. (1992). Emotions in work and achievement. Review of Industrial and Organizational Psychology, 7, 153-198. New York, NY: Wiley.

Pennsylvania Department of Corrections. (2016). Mental health services [Webpage]. Retrieved from http://www.cor.pa.gov/General\%20Information/Pages/Mental-HealthServices.aspx\#.WPgbrNLyu01

Petracek, C. (2012). Correctional officers' perceptions of working with inmates with mental illnesses and the effectiveness of mental health training. Retrieved from Sophia, the St. Catherine University repository website: https://sophia.stkate.edu/msw_papers/72

Petrides, K.V. \& Furnham, A. (2000). On the dimensional structure of emotional intelligence. Personality and Individual Differences, 29(2), 313-320. doi: 10.1016/s01918869(99)00195-6

Powers-Magro, C. (2015). Jail correctional officer's stress, job satisfaction, and perceptions of mentally ill inmates (Order No. 10119324). Available from ProQuest Dissertations \& Theses Global. (1805320785). Retrieved from http://www.libproxy.wvu.edu/login?url=https://search-proquestcom.www.libproxy.wvu.edu/docview/1805320785?accountid=2837

Primeau, A., Bowers, T. G., Harrison, M. A., \& Xuxu (2013). Deinstitutionalization of the mentally ill: Evidence for transinstitutionalization from psychiatric hospitals to penal institutions. Comprehensive Psychology, 2(1), 16-22. doi: 10.2466/16.02.13.cp.2.2

Prins, S. J. (2014). Prevalence of mental illnesses in US state prisons: A systematic review. Psychiatric Services, 65(7), 862-872. doi: 10.1176/appi.ps.201300166 
Reimann, R., Angleitner, A., \& Strelau, J. (1997). Genetic and environmental influences on personality: A study of twins reared together using the self-and peer report NEO-FFI scales. Journal of Personality, 65(3), 449-475. doi: 10.1111/j.1467-6494.1997.tb00324.x

Rieck, T., \& Callahan, J. L. (2013). Emotional intelligence and psychotherapy outcomes in the training clinic. Training and Education in Professional Psychology, 7(1), 42. doi:10.1037/a0031659

Rhodes, L. (2004). Total confinement: Madness and reason in the maximum-security prison. Berkeley, CA: University of California Press. doi: 10.2006-v30-n2-as1445/014141ar/

Robb, C., Haley, W., Becker, M., Polivka, L., \& Chwa, H. (2003). Attitudes towards mental health care in younger and older adults: Similarities and differences. Aging Mental Health, 7, 142-152. doi: 10.1080/1360786031000072321.

Rold, W. J. (2008). Thirty years after Estelle v. Gamble: A legal retrospective. Journal of Correctional Health Care, 14(1), 11-20. doi: 10.1177/1078345807309616

Saklofske, D. H., Austin, E. J., Galloway, J., \& Davidson, K. (2007). Individual difference correlates of health-related behaviours: Preliminary evidence for links between emotional intelligence and coping. Personality and Individual Differences, 42, 491-502. doi:10.1016/j.paid.2006.08.006

Sala, F. (2002). Emotional competence inventory (ECI): Technical manual. Boston, MA: Hay/Mcber Group.

Salovey, P., \& Mayer, J. D. (1990). Emotional intelligence. Imagination, Cognition and Personality, 9(3), 185-211. doi: 10.2190/dugg-p24e-52wk-6cdg

Salovey, P., Bedell, B., Detweiler, J. B., \& Mayer, J. D. (1999). Coping intelligently: Emotional intelligence and the coping process. In C. R. Snyder (Ed.), Coping: The psychology 
what works (pp. 141-164). New York, NY: Oxford University Press. doi:

10.1093/med:psych/9780195119343.001.0001

Sawyer, W. \& Wagner, P. (2020). Mass incarceration: The whole pie 2020 [Webpage].

Retrieved from https://www.prisonpolicy.org/reports/pie2020.html

Schaufeli, W. B., \& Peeters, M. C. (2000). Job stress and burnout among correctional officers: A literature review. International Journal of Stress Management, 7(1), 19-48. doi: 10.1023/a:1009514731657

Schutte, N. S., Malouff, J. M., Hall, L. E., Haggerty, D. J., Cooper, J. T., Golden, C. J., \& Dornheim, L. (1998). Development and validation of a measure of emotional intelligence. Personality and Individual Differences, 25(2), 167-177. doi: 10.1016/s01918869(98)00001-4

Schutte, N. S., \& Malouff, J. M. (2002). Incorporating emotional skills in a college transition course enhances student retention. Journal of the First-Year Experience and Students in Transition, 14, 7-21. doi:10.31274/etd-180810-3319

Schutte, N. S., Malouff, J. M., \& Thorsteinsson, E. B. (2013). Increasing emotional intelligence through training: Current status and future directions. International Journal of Emotional Education, 5(1), 56.

Segal, D., Coolidge, F., Mincic, M., \& O'Riley, A (2005). Beliefs about mental illness and willingness to seek help: A cross-sectional study. Aging Mental Health, 9, 363-367. doi: 10.1080/13607860500131047.

Shapiro, G., Cusi, A., Kirst, M., O'Campo, P., Nakhost, A., \& Stergiopoulos, V. (2015). Coresponding police-mental health programs: A review. Administration \& Policy In Mental 
Health \& Mental Health Services Research, 42(5), 606-620. doi:10.1007/s10488-0140594-9

Shenson, D., Dubler, N., \& Michaels, D. (1990). Jails and prisons: The new asylums? The American Journal of Public Health, 80(6), 655-656. doi: 10.2105/ajph.80.6.655

Shusman, E. J., \& Inwald, R. E. (1991). A longitudinal validation study of correctional officer job performance as predicted by the IPI and MMPI. Journal of Criminal Justice, 19(2), 173-180. doi:10.1016/0047-2352(91)90051-v

Skeem, J. L., Manchak, S., \& Peterson, J. K. (2011). Correctional policy for offenders with mental illness: Creating a new paradigm for recidivism reduction. Law and human behavior, 35(2), 110-126. doi: 10.1007/s10979-010-9223-7

Slaski, M., \& Cartwright, S. (2002). Health performance and emotional intelligence: An exploratory study of retail managers. Stress and Health, 18(2), 63-68. doi: 10.1002/smi.926

Slaski, M., \& Cartwright, S. (2003). EI training and its implications for stress health, and performance. Stress and Health, 19(4), 233-239. doi: 10.1002/smi.979

Skeem, J. L., Manchak, S., \& Peterson, J. K. (2011). Correctional policy for offenders with mental illness: Creating a new paradigm for recidivism reduction. Law and Human Behavior, 35(2), 110-126. doi: 10.1007/s10979-010-9223-7

Start, A. (1998). Interaction between correctional staff and health care providers in the delivery of medical care. Clinical Practice in Correctional Medicine, 26-31. doi: 10.2105/AJPH.2017.304248 
Steadman, H. J., Deane, M. W., Borum, R., \& Morrissey, J. P. (2000). Comparing outcomes of major models of police responses to mental health emergencies. Psychiatric Services, 51(5), 645-649. doi: 10.1176/appi.ps.51.5.645

Steadman, H. J. (1992). Boundary spanners: A key component for the effective interactions of the justice and mental health systems. Law and Human Behavior, 16(1), 75-87. doi: 10.1007/bf02351050

Steadman, H. J., McCarty, D. W., \& Morrissey, J. P. (1989). The mentally ill in jail: Planning for essential services. Guilford Press. doi: 10.1016/0047-2352(91)90086-b

Steadman, H.J., Osher, F.C., Robbins, P.C., Case, B., Samuels, S. (2009). Prevalence of serious mental illness among jail inmates. Psychiatric Services, 60(6), 761-765. doi: 10.1176/ps.2009.60.6.761

Stinneford, J. F. (2017). Against cruel innovation: The original meaning of the cruel and unusual punishment clause, and why that matters today [Webpage]. Retrieved from https://constitutioncenter.org/interactive-constitution/amendments/amendmentviii/against-cruel-innovation-the-original-meaning-of-the-cruel-and-unusualpuni/clause/10

Strelau, J. (1998). Temperament: A psychological perspective. Springer Science \& Business Media. doi: 10.4135/9780857025753.n199

Strelau, J. (1998). Temperament: A psychological perspective. New York, NY: Plenum. doi: 10.4135/9780857025753.n199

Stys, Y., \& Brown, S. L. (2004). A review of the emotional intelligence literature and implications for corrections. Research branch correctional service of Canada, 10(1), 168. doi: 10.4236/psych.2015.64042 
Spearlt, A. (2009). Mental illness in prison: Inmate rehabilitation \& correctional officers in crisis. Berkeley Journal of Criminal Law, 14(1), 277-280. doi: 10.15779/Z38JP6M

Spinaris, C. G., Denhof, M. D., \& Kellaway, J. A. (2012). Posttraumatic stress disorder in United States corrections professionals: Prevalence and impact on health and functioning. Florence, CO: Desert Waters Correctional Outreach [Webpage]. Retrieved from http://www.correctionsfatigue.com/images/PTSD_Prev_in_Corrections_2012.pdf

Sun, K. (2013). Correctional counseling: A cognitive growth perspective. Burlington, MA: Jones and Bartlett Learning. doi:10.1080/03601270500493974

Teller, J. L., Munetz, M. R., Gil, K. M., \& Ritter, C. (2006). Crisis intervention team training for police officers responding to mental disturbance calls. Psychiatric Services, 57(2), 232237. doi: 10.1176/appi.ps.57.2.232

Teitcher, J. E., Bockting, W. O., Bauermeister, J. A., Hoefer, C. J., Miner, M. H., \& Klitzman, R. L. (2015). Detecting, preventing, and responding to "fraudsters" in internet research: ethics and tradeoffs. The Journal of Law, Medicine \& Ethics, 43(1), 116-133.

Toker, S., \& Melamed, S. (2017). Stress, recovery, sleep, and burnout. The Handbook of Stress and Health, 168-185. doi: 10.1002/9781118993811.ch10

Torrey, E. F., Zdanowicz, M. T., Kennard, A. D., Lamb, H. R., Eslinger, D. F., Biasotti, M. I., Fuller, D. A. (2014). The treatment of persons with mental illness in prisons and jails: A state survey. Arlington, VA: Treatment Advocacy Center [Webpage]. Retrieved from https://www.treatmentadvocacycenter.org/storage/documents/treatment-behindbars/treatment-behind-bars.pdf

Torrey, E. F., Kennard, A. D., Eslinger, D. F., Lamb, H. R., Pavle, J. (2010). More mentally ill persons are in jails and prisons than hospitals: A survey of the states. Arlington, VA: 
Treatment Advocacy Center [Webpage]. Retrieved from https://www.treatmentadvocacycenter.org/storage/documents/final_jails_v_hospitals_stu dy.pdf

Totterdell, P., \& Holman, D. (2003). Emotion regulation in customer service roles: Testing a model of emotional labor. Journal of Occupational Health Psychology, 8, 55-73. doi:10.1037/1076-8998.8.1.55

Van Rooy, D. L., \& Viswesvaran, C. (2004). Emotional intelligence: A meta-analytic investigation of predictive validity and nomological net. Journal of Vocational Behavior, 65(1), 71-95. doi: 10.1016/s0001-8791(03)00076-9

Visher, C. A. (2007). Returning home: Emerging findings and policy lessons about prisoner re-entry. Federal Sentencing Reporter 20(1), 93-102. doi: 10.1525/fsr.2007.20.2.93

Waldron, V. R., \& Krone, K. J. (1991). The experience and expression of emotion in the workplace: A study of a corrections organization. Management Communication Quarterly, 4(3), 287-309. doi: 10.1177/0893318991004003002

Weber Brooks, L., \& Leeper Piquero, N. (1998). Police stress: does department size matter? Policing: An International Journal of Police Strategies \& Management, 21(4), 600-617. doi: $10.1108 / 13639519810241647$

Wechsler, D. (1958). The measurement and appraisal of adult intelligence. Baltimore, MD: Williams \& Wilkins. doi: 10.1037/11167-000

Whitehead, J. T., \& Lindquist, C. A. (1986). Correctional officer job burnout: A path model. Journal of Research in Crime and Delinquency, 23(1), 23-42. doi: $10.1177 / 0022427886023001003$ 
Winters, R. (2014). Understanding and enhancing the value of female corrections professionals [Webpage]. Retrieved from http://www.corrections.com/news/article/36734understanding-and-enhancing-the-value-of-female-corrections-professionals 


\section{APPENDICES}

\section{Appendix A: Informed Consent}

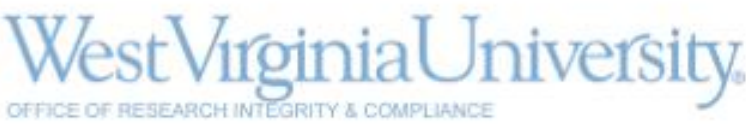

OTFCE OF RESEARCH INTEGRTY S COMPLIANCE
Human Research Protocol Only Minimal Risk Consent Form

Without HIPAA

\section{Only Minimal Risk \\ Consent Information Form (without HIPAA)}

Principal Investigator

Department

Psychology

Protocol Number

Study Title

Relationship

Co-Investigator(s)
Courtney N. Hull

Department of Counseling, Rehabilitation Counseling, and Counseling

Correctional Officer Attitudes toward Offenders with Mental Illness: The

des, Burnout, Emotional Intelligence, and Mental Health Training

Jeffrey Daniels, Ph.D. and Christine Schimmel, Ed.D.

\section{Contact Persons}

In the event you experience any side effects or injury related to this research, you should contact Dr. Jeffrey Daniels at (304)-293-2235 or Dr. Christine Schimmel at (304) 293-2266. If you have any questions, concerns, or complaints about this research, you can contact Dr. Christine Schimmel (304) 293-2266, Dr. Daniels (304)293-2235, or Courtney Hull at (812) 251-8980.

For information regarding your rights as a research subject, to discuss problems, concerns, or suggestions related to the research, to obtain information or offer input about the research, contact the Office of Research Integrity and Compliance at (304) 293-7073. In addition, if you would like to discuss problems, concerns, have suggestions related to research, or would like to offer input about the research, contact the Office of Research Integrity and Compliance at 304-293-7073. In addition, if you would like to discuss problems, concerns, have suggestions related to research, or would like to offer input about the research, contact the Office of Research Integrity and Compliance at 304-293-7073.

\section{Introduction}

This study is being conducted by Courtney Hull, M.S. and supervised by Jeffrey Daniels, Ph.D. and Christine Schimmel, Ed.D., in the Department of Counseling, Rehabilitation Counseling, and Counseling Psychology at West Virginia University.

\section{Purpose(s) of the Study}


The purpose of this study is to identify correctional officer attitudes toward offenders with mental illness, and to understand the relationship between officer attitudes, burnout, and emotional intelligence. Additionally, this study aims to understand if mental health training for correctional officers can lead to higher emotional intelligence, more positive attitudes, and lower levels of burnout. This information will be useful for mental health professionals and administrative personnel to better understand the dynamic interplay between officers and offenders who suffer from SMI. This consequently assists mental health professionals and facility administrators in working effectively as part of interdisciplinary teams and in determining interventions and training modalities for correctional officers.

\section{Description of Procedures}

This study involves answering a few demographic questions about yourself, and then answering a longer survey. It will take approximately 20 minutes for you to complete. You will be asked to fill out a questionnaire regarding you attitudes toward offenders with mental illness. Additionally, you will be asked questions regarding burnout and emotional intelligence in the context of your work environment. You do not have to answer all of the questions.

\section{Discomforts}

There are no known or expected risks from participating in this study.

\section{Benefits}

You may choose to provide your email address to receive 1 of 120, \$10 Amazon ${ }^{\mathrm{TM}}$ gift cards. Should you choose to provide your email it will not be linked in any way to your questionnaire responses.

\section{Financial Considerations}

There are no special fees for participating in this study.

\section{Confidentiality}

Any information about you that is obtained as a result of your participation in this research will be kept as confidential as legally possible. Your research records and test results, just like hospital records, may be subpoenaed by court order or may be inspected by the study sponsor or federal regulatory authorities (including the FDA if applicable) without your additional consent.

In addition, there are certain instances where the researcher is legally required to give information to the appropriate authorities. These would include mandatory reporting of infectious diseases, mandatory reporting of information about behavior that is imminently dangerous to your child or to others, such as suicide, child abuse, etc.

In any publications that result from this research, neither your name nor any information from which you might be identified will be published without your consent.

\section{Voluntary Participation}


Participation in this study is voluntary. You are free to withdraw your consent to participate in this study at any time by simply closing the window.

Refusal to participate or withdrawal will result in no penalty to you.

I willingly agree to be in the study.
o Yes
$\circ$ No 


\section{Appendix B: Participant Invitation Request (Social Media)}

(Post to be disseminated on social media platforms)

Correctional officer:

My name is Courtney Hull, and I am a doctoral candidate in counseling psychology in the College of Education and Human Services at West Virginia University. I am hoping to collect data for my dissertation project which is exploring correctional officer attitudes toward offenders with mental illness, and the relationships between attitudes, burnout, and emotional intelligence. The project is supervised by Dr. Jeffrey Daniels Ph.D., Department Chairperson and Associate Professor, and Dr. Christine Schimmel Ed.D, Assistant Department Chairperson, Associate Professor and Coordinator of the School Counseling Program.

Your participation in this project is greatly appreciated and will take approximately 10 minutes to fill out the attached questionnaire. You will have the opportunity to enter to win one of $120, \$ 10$ gift cards to Amazon ${ }^{\mathrm{TM}}$ as a thank you for your participation! We ask that you complete the survey by [date]. To enter to win a gift card, just click the link provided on the last page of the survey and provide your email. Please note, for your privacy, email addresses are not linked with your survey results.

Your involvement in this project will be kept as private as legally possible. All data will be reported as a collective. You must be 18 years of age or older and currently employed as a correctional officer. We will not ask any information that should lead back to your identity as a participant. Your participation is completely voluntary. You may skip any question that you do not wish to answer, and you may discontinue at any time. West Virginia University's Institutional Review Board approval of this research project is on file.

We hope that you will participate in this research project, as it will be beneficial in understanding relevant attitudes and experiences of correctional officers who have frequent contact with mentally ill offenders in prisons. Thank you very much for your time. Should you have any questions about this letter or the research project, please feel free to contact Courtney Hull at cnh0008@mix.wvu.edu. 
If you are willing to participate in this study, please click the link below:

Sincerely, Courtney Hull, M.S. 


\section{Appendix C: Two Week Participation Invitation Follow-Up (Social Media)}

\section{Correctional Officer:}

Approximately two weeks ago, I made a post asking for your participation in a survey examining correctional officer attitudes toward offenders with mental illness and the relationships between attitudes, emotional intelligence, burnout, and mental health training. Our hope is to survey as many correctional officers as possible.

Again, I appreciate that your time is limited and ask you click on the following URL to be taken to the 10-minute survey:

If you find that the above link does not work, you may copy and paste it to your browser. You will have the opportunity to enter to win one of $120, \$ 10$ gift cards to Amazon ${ }^{\mathrm{TM}}$ as a thank you for your participation! We ask that you complete the survey by [date]. To enter to win a gift card, just click the link provided on the last page of the survey and provide your email. Please note, for your privacy, email addresses are not linked with your survey results. Thank you for your time and willingness to participate in this study.

Sincerely, Courtney Hull, M.S. 


\section{Appendix D: Four Week Participation Invitation Follow-Up (Social Media)}

Correctional Officer:

Approximately four weeks ago, I made a post asking for your participation in a survey examining correctional officer attitudes toward offenders with mental illness and the relationships between attitudes, emotional intelligence, burnout, and mental health training. Our hope is to survey as many correctional officers as possible.

Again, I appreciate that your time is limited and ask you click on the following URL to be taken to the 10-minute survey:

If you find that the above link does not work, you may copy and paste it to your browser. You will have the opportunity to enter to win one of $120, \$ 10$ gift cards to Amazon ${ }^{\mathrm{TM}}$ as a thank you for your participation! We ask that you complete the survey by [date]. To enter to win a gift card, just click the link provided on the last page of the survey and provide your email. Please note, for your privacy, email addresses are not linked with your survey results. Thank you for your time and willingness to participate in this study.

Sincerely, Courtney Hull, M.S. 


\section{Appendix E: Post-Study Information}

Thank you for your participation in this study! Your responses to the survey questions are greatly appreciated.

If you would like to be redirected to an external link for the opportunity to enter a drawing for one of 120, \$10, electronic Amazon ${ }^{\mathrm{TM}}$ gift cards, please click here.

\section{Anonymity and Confidentiality:}

If you choose to enter the drawing for one of several small, Amazon ${ }^{\mathrm{TM}}$ gift cards, please be aware that your email address as having completed the study will be provided to the researcher. While your email address will not be directly associated with your answers, complete anonymity is not possible due to entering the drawing; instead your answers still remain strictly confidential and will be secured stored.

If you choose not to enter the drawing from one of several small, Amazon ${ }^{\mathrm{TM}}$ gift cards, your responses are completely anonymous as no identifying information has been requested from you. In addition, the secure survey software (Qualtrics) that collected your responses has been programmed to anonymize all data by removing respondents' IP addresses.

In order to maintain the quality of this study, please do not disclose research procedures to anyone who might participate in this study in the future as this could bias the results.

\section{Final Report:}

If you would like to receive a copy of a summary of the findings of this study when it is completed, please feel free to contact me at cnh0008@mix.wvu.edu. 


\section{Appendix F: Demographic Questionnaire}

1. Are you currently employed as a correctional officer? (You must be currently employed as a correctional officer to participate in the study)
a. Yes
b. No

2. Please type in your age. (You must be 18 years of age or older to participate)

3. Which of the following best describes your gender?
a. Male
b. Female
c. Other (Please Specify):

4. How long have you been employed as a correctional officer? Please type a number in years.

5. At what type of institution are you currently employed?
a. State Prison
b. Federal Prison
c. Other:

6. Approximately how many hours of specialized mental health training have you received since you began working as a correctional officer? (Separate from pre-service training) 


\section{Appendix G: Opinions About Mental Illness Scales}

(Cohen \& Struening, 1962)

The statements that follow are opinions or ideas about mental illness and mentally ill patients (inmates). There are many differences of opinion about this subject. In other words, many people agree with each of the following statements while many people disagree with each of the statements. We would like to know what you think about these statements. Please Check box that best describes how you feel about each statement. There are no right or wrong answers; we are interested only in your opinion. It is very important that you answer every item.

\begin{tabular}{|c|c|c|c|c|c|c|}
\hline & $\begin{array}{l}\text { Strongly } \\
\text { Agree }\end{array}$ & Agree & $\begin{array}{l}\text { Partly } \\
\text { Agree }\end{array}$ & $\begin{array}{l}\text { Partly } \\
\text { Disagree }\end{array}$ & Disagree & $\begin{array}{l}\text { Strongly } \\
\text { Disagree }\end{array}$ \\
\hline $\begin{array}{l}\text { 1. Nervous breakdowns } \\
\text { usually result when people } \\
\text { work too hard. }\end{array}$ & & & & & & \\
\hline $\begin{array}{l}\text { 2. Although patients } \\
\text { discharged from mental } \\
\text { hospitals may seem all } \\
\text { right, they should not be } \\
\text { allowed to marry. }\end{array}$ & & & & & & \\
\hline $\begin{array}{l}\text { 3. It is easy to recognize } \\
\text { someone who once had a } \\
\text { serious mental illness. }\end{array}$ & & & & & & \\
\hline $\begin{array}{l}\text { 4. People who are mentally } \\
\text { ill let their emotions control } \\
\text { them: normal people think } \\
\text { things out. }\end{array}$ & & & & & & \\
\hline $\begin{array}{l}\text { 5. People who were once } \\
\text { patients in mental hospitals } \\
\text { are no more dangerous than } \\
\text { the average citizen. }\end{array}$ & & & & & & \\
\hline $\begin{array}{l}\text { 6. When a person has a } \\
\text { problem or a worry, it is } \\
\text { best to think about it, but } \\
\text { keep busy with more } \\
\text { pleasant things. }\end{array}$ & & & & & & \\
\hline
\end{tabular}




\begin{tabular}{|c|c|c|c|c|c|c|}
\hline & $\begin{array}{l}\text { Strongly } \\
\text { Agree }\end{array}$ & Agree & $\begin{array}{l}\text { Partly } \\
\text { Agree }\end{array}$ & $\begin{array}{l}\text { Partly } \\
\text { Disagree }\end{array}$ & Disagree & $\begin{array}{l}\text { Strongly } \\
\text { Disagree }\end{array}$ \\
\hline $\begin{array}{l}\text { 7. There is something about } \\
\text { mental patients that makes } \\
\text { it easy to tell them from } \\
\text { normal people. }\end{array}$ & & & & & & \\
\hline $\begin{array}{l}\text { 8. The small children of } \\
\text { patients in mental hospitals } \\
\text { should not be allowed to } \\
\text { visit them. }\end{array}$ & & & & & & \\
\hline $\begin{array}{l}\text { 9. People would not become } \\
\text { mentally ill if they avoided } \\
\text { bad thoughts. }\end{array}$ & & & & & & \\
\hline $\begin{array}{l}\text { 10. A heart patient has just } \\
\text { one thing wrong with him, } \\
\text { while a mentally ill person } \\
\text { is completely different from } \\
\text { other patients. }\end{array}$ & & & & & & \\
\hline $\begin{array}{l}\text { 11. People with mental } \\
\text { illness should never be } \\
\text { treated in the same hospital } \\
\text { with people with physical } \\
\text { illness. }\end{array}$ & & & & & & \\
\hline $\begin{array}{l}\text { 12. A woman would be } \\
\text { foolish to marry a man who } \\
\text { had a severe mental illness, } \\
\text { even though he seems fully } \\
\text { recovered. }\end{array}$ & & & & & & \\
\hline $\begin{array}{l}\text { 13. Anyone who is in a } \\
\text { hospital for a mental illness } \\
\text { should not be allowed to } \\
\text { vote. }\end{array}$ & & & & & & \\
\hline
\end{tabular}




\begin{tabular}{|c|c|c|c|c|c|c|}
\hline & $\begin{array}{l}\text { Strongly } \\
\text { Agree }\end{array}$ & Agree & $\begin{array}{l}\text { Partly } \\
\text { Agree }\end{array}$ & $\begin{array}{l}\text { Partly } \\
\text { Disagree }\end{array}$ & Disagree & $\begin{array}{l}\text { Strongly } \\
\text { Disagree }\end{array}$ \\
\hline $\begin{array}{l}\text { 14. Mental illness is usually } \\
\text { caused by some disease of } \\
\text { the nervous system. }\end{array}$ & & & & & & \\
\hline $\begin{array}{l}\text { 15. Most women who were } \\
\text { once patients in a mental } \\
\text { hospital could be trusted as } \\
\text { baby-sitters. }\end{array}$ & & & & & & \\
\hline $\begin{array}{l}\text { 16. Most patients in mental } \\
\text { hospitals don't care how } \\
\text { they look. }\end{array}$ & & & & & & \\
\hline $\begin{array}{l}\text { 17. College professors are } \\
\text { more likely to become } \\
\text { mentally ill than are } \\
\text { businessmen. }\end{array}$ & & & & & & \\
\hline $\begin{array}{l}\text { 18. Although some mental } \\
\text { patients seem all right, it is } \\
\text { dangerous to forget for a } \\
\text { moment that they are } \\
\text { mentally ill. }\end{array}$ & & & & & & \\
\hline $\begin{array}{l}\text { 19. Sometimes mental } \\
\text { illness is punishment for } \\
\text { bad deeds. }\end{array}$ & & & & & & \\
\hline $\begin{array}{l}\text { 20. One of the main causes } \\
\text { of mental illness is a lack of } \\
\text { moral strength or will } \\
\text { power. }\end{array}$ & & & & & & \\
\hline $\begin{array}{l}\text { 21. All patients in mental } \\
\text { hospitals should be } \\
\text { prevented from having } \\
\text { children by a painless } \\
\text { operation }\end{array}$ & & & & & & \\
\hline
\end{tabular}




\section{Appendix H: Schutte Self-Report Emotional Intelligence Test}

(Schutte, et al., 1998)

Each of the following items asks you about your emotions or reactions associated with emotions After deciding whether a statement is generally true for you, use the 5-point scale to respond to the statement. Please circle the " 1 " if you strongly disagree that this is like you, the " 2 " if you somewhat disagree that this is like you, " 3 " if you neither agree nor disagree that this is like you, the " 4 " if you somewhat agree that this is like you. and the " 5 " if you strongly agree that this is like you. There are no right or wrong answers Please give the response that best describes you.

$$
1=\text { strongly disagree } \quad 2=\text { somewhat disagree } \quad 3=\text { neither agree nor disagree }
$$

$$
4=\text { somewhat agree } \quad 5=\text { strongly agree }
$$

\begin{tabular}{|c|c|c|c|c|c|}
\hline $\begin{array}{l}\text { 1. I know when to speak about my personal } \\
\text { problems to others. }\end{array}$ & 1 & 2 & 3 & 4 & 5 \\
\hline $\begin{array}{l}\text { 2. When I am faced with obstacles, I } \\
\text { remember times I faced similar obstacles } \\
\text { and overcame them. }\end{array}$ & 1 & 2 & 3 & 4 & 5 \\
\hline $\begin{array}{l}\text { 3. I expect that I will do well on most things } \\
\text { I try. }\end{array}$ & 1 & 2 & 3 & 4 & 5 \\
\hline 4. Other people find it easy to confide in me. & 1 & 2 & 3 & 4 & 5 \\
\hline $\begin{array}{l}\text { 5. I find it hard to understand the non-verbal } \\
\text { messages of other people. }\end{array}$ & 1 & 2 & 3 & 4 & 5 \\
\hline $\begin{array}{l}\text { 6. Some of the major events of my life have } \\
\text { led me to re-evaluate what is important } \\
\text { and not important. }\end{array}$ & 1 & 2 & 3 & 4 & 5 \\
\hline $\begin{array}{l}\text { 7. When my mood changes, I see new } \\
\text { possibilities. }\end{array}$ & 1 & 2 & 3 & 4 & 5 \\
\hline $\begin{array}{l}\text { 8. Emotions are one of the things that make } \\
\text { my life worth living. }\end{array}$ & 1 & 2 & 3 & 4 & 5 \\
\hline $\begin{array}{l}\text { 9. I am aware of my emotions as I } \\
\text { experience them. }\end{array}$ & 1 & 2 & 3 & 4 & 5 \\
\hline 10. I expect good things to happen. & 1 & 2 & 3 & 4 & 5 \\
\hline 11. I like to share my emotions with others. & 1 & 2 & 3 & 4 & 5 \\
\hline
\end{tabular}




\begin{tabular}{|c|c|c|c|c|c|}
\hline $\begin{array}{l}\text { 12. When I experience a positive emotion, I } \\
\text { know how to make it last. }\end{array}$ & 1 & 2 & 3 & 4 & 5 \\
\hline 13. I arrange events others enjoy. & 1 & 2 & 3 & 4 & 5 \\
\hline 14. I seek out activities that make me happy. & 1 & 2 & 3 & 4 & 5 \\
\hline $\begin{array}{l}\text { 15. I am aware of the non-verbal messages I } \\
\text { send to others. }\end{array}$ & 1 & 2 & 3 & 4 & 5 \\
\hline $\begin{array}{l}\text { 16. I present myself in a way that makes a } \\
\text { good impression on others. }\end{array}$ & 1 & 2 & 3 & 4 & 5 \\
\hline $\begin{array}{l}\text { 17. When I am in a positive mood, solving } \\
\text { problems is easy for me. }\end{array}$ & 1 & 2 & 3 & 4 & 5 \\
\hline $\begin{array}{l}\text { 18. By looking at their facial expressions, I } \\
\text { recognize the emotions people are } \\
\text { experiencing. }\end{array}$ & 1 & 2 & 3 & 4 & 5 \\
\hline 19. I know why my emotions change. & 1 & 2 & 3 & 4 & 5 \\
\hline $\begin{array}{l}\text { 20. When I am in a positive mood, I am able } \\
\text { to come up with new ideas. }\end{array}$ & 1 & 2 & 3 & 4 & 5 \\
\hline 21. I have control over my emotions. & 1 & 2 & 3 & 4 & 5 \\
\hline $\begin{array}{l}\text { 22. I easily recognize my emotions as I } \\
\text { experience them. }\end{array}$ & 1 & 2 & 3 & 4 & 5 \\
\hline $\begin{array}{l}\text { 23. I motivate myself by imagining good } \\
\text { outcomes to tasks I take on. }\end{array}$ & 1 & 2 & 3 & 4 & 5 \\
\hline $\begin{array}{l}\text { 24. I complement others when they have done } \\
\text { something well. }\end{array}$ & 1 & 2 & 3 & 4 & 5 \\
\hline $\begin{array}{l}\text { 25. I am aware of the non-verbal messages } \\
\text { other people send. }\end{array}$ & 1 & 2 & 3 & 4 & 5 \\
\hline $\begin{array}{l}\text { 26. When another person tells me about an } \\
\text { important event in his or her life, I almost } \\
\text { feel as though I experienced this event } \\
\text { myself. }\end{array}$ & 1 & 2 & 3 & 4 & 5 \\
\hline $\begin{array}{l}\text { 27. When I feel a change in emotions, I tend } \\
\text { to come up with new ideas. }\end{array}$ & 1 & 2 & 3 & 4 & 5 \\
\hline $\begin{array}{l}\text { 28. When I am faced with a challenge, I give } \\
\text { up because I believe I will fail. }\end{array}$ & 1 & 2 & 3 & 4 & 5 \\
\hline $\begin{array}{l}\text { 29. I know what other people are feeling just } \\
\text { by looking at them. }\end{array}$ & 1 & 2 & 3 & 4 & 5 \\
\hline $\begin{array}{l}\text { 30. I help other people feel better when they } \\
\text { are down. }\end{array}$ & 1 & 2 & 3 & 4 & 5 \\
\hline
\end{tabular}




\begin{tabular}{|l|llllll|}
\hline $\begin{array}{l}\text { 31. I use good moods to help myself keep } \\
\text { trying in the face of obstacles. }\end{array}$ & 1 & 2 & 3 & 4 & 5 \\
\hline $\begin{array}{l}\text { 32. I can tell how people are feeling by } \\
\text { listening to the tone of their voice. }\end{array}$ & 1 & 2 & 3 & 4 & 5 \\
\hline $\begin{array}{l}\text { 33. It is difficult for me to understand why } \\
\text { people feel the way they do. }\end{array}$ & 1 & 2 & 3 & 4 & 5 \\
\hline
\end{tabular}




\section{Appendix I: Copenhagen Burnout Inventory}

(Kristensen, Borritz, Villadsen, \& Christensen, 2005).

\section{Part One: Personal burnout}

Definition: Personal burnout is a state of prolonged physical and psychological exhaustion.

Scoring: Always: 100. Often: 75. Sometimes: 50. Seldom: 25. Never/Almost Never: 0. Total score on the scale is the average of the scores on the items. If less than three questions have been answered, the respondent is classified as non-responder.

\section{Questions:}

1. How often do you feel tired?
a) Always
b) Often
c) Sometimes
d) Seldom
e) Never

2. How often are you physically exhausted?
a) Always
b) Often
c) Sometimes
d) Seldom
e) Never

3. How often are you emotionally exhausted?
a) Always
b) Often
c) Sometimes
d) Seldom
e) Never

4. How often do you think: "I can't take it anymore"?
a) Always
b) Often
c) Sometimes
d) Seldom
e) Never

5. How often do you feel worn out? 

a) Always
b) Often
c) Sometimes
d) Seldom
e) Never

6. How often do you feel weak and susceptible to illness?
a) Always
b) Often
c) Sometimes
d) Seldom
e) Never

\section{Part Two: Work-related burnout}

Definition: Work-related burnout is a state of prolonged physical and psychological exhaustion, which is perceived as related to the person's work.

Three first questions: To a very high degree, To a high degree, Somewhat, To a low degree, To a very low degree.

Last four questions: Always, Often, Sometimes, Seldom, Never/almost never. Reversed score for last question.

Scoring as for the first scale. If less than four questions have been answered, the respondent is classified as non-responder.

\section{Questions:}

1. Is your work emotionally exhausting?

a) To a very high degree

b) To a high degree

c) Somewhat

d) To a low degree

e) To a very low degree

2. Do you feel burnt out because of your work?
a) To a very high degree
b) To a high degree
c) Somewhat
d) To a low degree
e) To a very low degree

3. Does your work frustrate you? 

a) To a very high degree
b) To a high degree
c) Somewhat
d) To a low degree
e) To a very low degree

4. Do you feel worn out at the end of the working day?
a) Always
b) Often
c) Sometimes
d) Seldom
e) Never

5. Are you exhausted in the morning at the thought of another day at work?
a) Always
b) Often
c) Sometimes
d) Seldom
e) Never

6. Do you feel that every working hour is tiring for you?
a) Always
b) Often
c) Sometimes
d) Seldom
e) Never

7. Do you have enough energy for family and friends during leisure time?
a) Always
b) Often
c) Sometimes
d) Seldom
e) Never 


\section{Part Three: Client-related burnout}

Definition: Client-related burnout is a state of prolonged physical and psychological exhaustion, which is perceived as related to the person's work with clients*.

*Clients, patients, social service recipients, elderly citizens, or inmates.

The four first questions: To a very high degree, To a high degree, Somewhat, To a low degree, To a very low degree.

The two last questions: Always, Often, Sometimes, Seldom, Never/almost never.

Scoring as for the first two scales. If less than three questions have been answered, the respondent is classified as non-responder.

\section{Questions:}

1. Do you find it hard to work with inmates?

a) To a very high degree

b) To a high degree

c) Somewhat

d) To a low degree

e) To a very low degree

2. Do you find it frustrating to work with inmates?

a) To a very high degree

b) To a high degree

c) Somewhat

d) To a low degree

e) To a very low degree

3. Does it drain your energy to work with inmates?

a) To a very high degree

b) To a high degree

c) Somewhat

d) To a low degree

e) To a very low degree

4. Do you feel that you give more than you get back when you work with inmates?
a) To a very high degree
b) To a high degree
c) Somewhat
d) To a low degree
e) To a very low degree 
5. Are you tired of working with inmates?
a) Always
b) Often
c) Sometimes
d) Seldom
e) Never

6. Do you sometimes wonder how long you will be able to continue working with inmates?
a) Always
b) Often
c) Sometimes
d) Seldom
e) Never 


\section{Appendix J: Acknowledgement of Exemption}

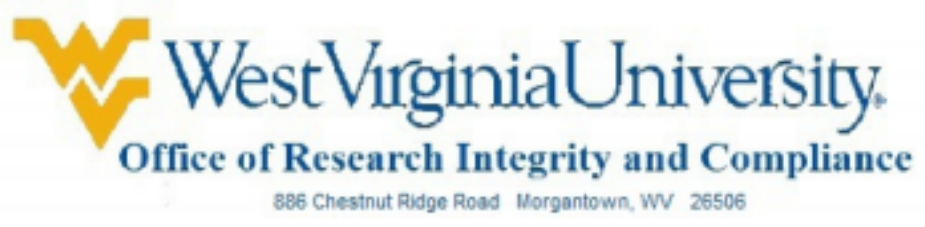

\section{Acknowledgement of Exemption}

$09 / 26 / 2019$

To: Jeffrey Daniels

From: WVU Office of Research Integrity \& Compliance

Protocol Type: Exempt

Submission Type: Initial

Funding: N/A

WVU Protocol \#: 1908686391

Protocol Title: Correctional Officer Attitudes toward Offenders with Mental Illness: The Relationship between Attitudes, Burnout, Emotional Intelligence, and Mental Health Training
Approval Date: 09/26/2019

Expiration Date: 09/25/2024

The West Virginia University Institutional Review Board has reviewed your submission of Exempt protocol 1908686391. Additional details regarding the review are below:

- This research study was granted an exemption because the Research involves educational tests, survey procedures, interview procedures or observation of public behavior and (i) information obtained is recorded in such a manner that human subjects cannot be identified, directly or through identifiers linked to the subjects; and (ii) any disclosure of the human subjects responses outside the research could not reasonably place the subjects at risk of criminal or civil liability or be damaging to the subjects financial standing, employability, or reputation [45 CFR 46.101(2)]. All exemptions are only good for three years. If this research extends more than three years beyond the approved date, then the researcher will have to request another exemption. The following documents have been acknowledged for use in this study and are available in the WVU+ke system:

The following documents were reviewed and approved for use as part of this submission. Only the documents listed below may be used in the research. Please aceess and print the files in the Notes \& Attachments section of your approved protocol.

- Recruitment Letter.docx

Protocol 190868639 i

FWA: 00005078

IORG: 0000194
Fhone: 304-293-7073

Fax: 304-293-3098

Email: IRBgmail.wvu.edu 
- Services Referral List.docx

- Copenhagen Bumout Inventory Appendix I.doex

- Demographic Questionnaire Appendix F.docx

- OMI Scale Appendix G.docx

- Schutte Permission Email.PNG

- Schutte Self Report Emotional Intelligence Test Appendix H.docx

- SSEIT permission request letterdocx

- Post-Study Information Letter.docx

- Four Week Participation Reminder.docx

- Two Week Participation Reminder.docx

\section{WVU IRB acknowledgement of protocol 1908686391 will expire on 09/25/2024.}

If the study is to continue beyond the expiration date, a renewal application must be submitted no later than two (2) weeks prior to expiration date. It is your responsibility to submit your protocol for renewal.

Once you begin your human subjects research, the following regulations apply:

1. Unanticipated or serious adverse events and/or side effects encountered in this research study must be reported to the IRB within five (5) days, using the Notify IRB action in the electronic protocol.

2. Any modifications to the study protocol should be submitted only if there will be an increase in risk to subjects accompanying the proposed change(s).

3. You may not use a modified information sheet until it has been reviewed and acknowledged by the WVU IRB prior to implementation.

The Office of Research Integrity and Compliance will be glad to provide assistance to you throughout the research process. Please feel free to contact us by phone, at 304.293 .7073 or by email at IRB amail.wvu.edu.

Sincerely,

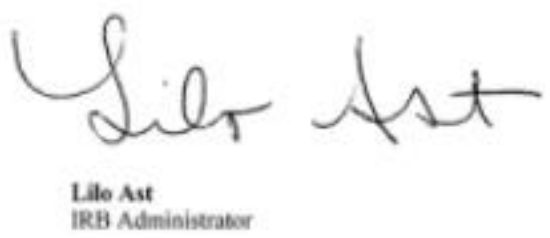




\section{Appendix K: IRB Approval of Protocol Amendment}

\section{WestVirginiaUniversity. \\ OFFICE OF HUMAN RESEARCH PROTECTIONS \\ 836 Chestrut Ridge foad Morgantom, WV 26506}

\section{Approval of Protocol Amendment}

$03 / 03 / 2020$

To: Jeffrey Daniels

From: WVU Human Research Protection Program

Protocol Type: Exempt

Submission Type: Amendment

Funding: N/A

WVU Protocol \#: 1908686391A001

Protocol Title: Correctional Officer Attitudes toward Offenders with Mental Illness: The Relationship between Attitudes, Burnout, Emotional Intelligence, and Mental Health Training

The West Virginia University Institutional Review Board has reviewed your request for modifications to the Exempt protocol 1908686391A001, in accordance with 45 CFR 46, 21 CFR 50, and 21 CFR 56 (when applicable). The amendment wording is as follows:

- After initial data collection through the online survey on social media sites, the data was found to be corrupt based on the following information: The data set contains 112 total participants and one survey preview 12 of the participants did not complete the survey, making the number of participants who completed the survey in it entirety 100. A large portion of the data set provided inconsistent responses to questions regarding age and length of employment as a correctional officer (ex. 19 years of age, employed as a correctional officer for 20 years; 28 years of age, employed as a correctional officer for 17 years; 20 years of age, employed as a correctional officer for 11 years, etc.) 99 of the 100 cases show evidence of corruption. The evidence is as follows: 099 of the 100 cases provided identical responses to all survey items. The data set is nearly entirely uniform. 099 of the 100 cases responded that they are employed in state correctional facilities. There is no variation in the sample. 099 of the 100 cases provided Gmail email addresses for incentives. Email addresses appear to be randomly generated or odd (ex. picturemxhg@gmail.com; extendwhqs@gmail.com; approachotuf@gmail.com; favorablemvru@gmail.com; uncoverxvfc@gmail.com) To address the aforementioned corruption concems, the following amendments will be made to the protocol: A CAPTCHA (Completely Automated Public Turing test to Tell Computers and Humans Apart) verification will be added to the Qualtrics survey as a security check to ensure that only human participants can continue with the survey. Participants will only be recruited through closed 
correctional officer social media groups to ensure that the survey reaches the targeted population. Survey responses will be closely monitored to ensure that the data collected is not corrupt. This will be accomplished by lowering the survey to 25 and collecting data in 25 participant increments. The following language will be added to the recruitment letter to deter data corruption: If the response set is found to be fraudulent, or you are found to have taken the survey more than once, you will be ineligible to be entered into the drawing for a gift card. The paragraph in the recruitment letter regarding incentives will be changed to the following: Your participation in this project is greatly appreciated and will take approximately 10 minutes to fill out the attached questionnaire. You will have the opportunity to be entered into a drawing to win one of $50, \$ 10$ gift cards to Amazon(TM) as a thank you for your participation! We ask that you complete the survey by [date]. To enter to win a gift card, just click the link provided on the last page of the survey and provide your email. Please note, for your privacy, email addresses are not linked with your survey results.

The following documents were reviewed and approved for use as part of this submission. Only the documents listed below may be used in the research. Please access and print the files in the Notes \& Attachments section of your approved protocol.

- IRB Amendment.docx

- wVU Unanticipated Event Form.docx

- Recruitment Letter.docx

- Services Referral List.docx

- Copenhagen Burnout Inventory Appendix Idocx

- Demographic Questionnaire Appendix Fdocx

- OMI Scale Appendix G.docx

- Schutte Permission Email.PNG

- Schutte Self Report Emotional Intelligence Test Appendix Hdocx

- SSEIT permission request letter.docx

- Post-Study Information Letter docx

- Four Week Participation Reminderdocx

- Two Week Participation Reminder,docx

WVU IRB approval of this amendment does not extend the approval period. The protocol retains the expiration date of $09 / 25 / 2024$

As you continue your human subjects research, the following regulations apply:

1. Unanticipated, serious adverse events and/or side effect(s) encountered at WVU or an affiliate site that are relakd to the research must be reported to the WVU IRB within five (5) days using the Notify IRB action in WVU+ke.

2. Any Unanticipated Problem or UPIRTSO or other research related event resulting in new or increased risk of harm to study subjects, occurring at WVU or an affiliate site, must be reported to the WVU IRB within five (5) days using the Notify IRB action in WVU+ke. 
3. Any modifications to the protocol or informed consent form must be reviewed and approved by the IRB priar to implementation. These modifications should be submitted as an amendment.

4. You may not use a modified informed consent form until it has been reviewod and approved by the WVU IRB. Only consent forms with the WVU+ke water mark may be used to obtain informed consent from participants.

The WVU Human Research Protection Program will be glad to provide assistance to you throughout the research process. Please feel free to contact us by phone at 304.293 .7073 or by email at IRB@mail.wyu.edu.

Sincerely,

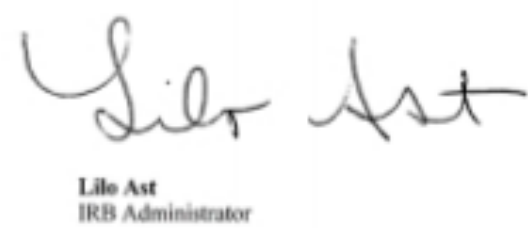




\section{Appendix L: Letter Requesting Access to the SSEIT}

Nicola Schutte, Ph.D.

School of Psychology

University of New England

Armidale NSW 2351, Australia

Dear Dr. Schutte,

My name is Courtney Hull, and I am currently a doctoral candidate in counseling psychology at West Virginia University. I am developing a dissertation proposal titled Correctional Officer Attitudes toward Offenders with Mental Illness: The Relationship between Attitudes, Burnout, Emotional Intelligence, and Mental Health Training. The purpose of this study is to identify correctional officer attitudes toward offenders with mental illness, and to understand the relationship between officer attitudes, burnout, and emotional intelligence. Additionally, this study aims to understand if mental health training for correctional officers can lead to higher emotional intelligence, more positive attitudes, and lower levels of burnout. This information will be useful for mental health professionals and administrative personnel to better understand the dynamic interplay between officers and offenders who suffer from SMI.

I am currently preparing to collect data for this project. I am writing to you to request permission to include in this research the Schutte Self-Report Emotional Intelligence Test, which I believe you hold the copyright. I would greatly appreciate your granting me permission to access and include the SSEIT in my project. If you have any questions or concerns about this research, please contact the project chair, Dr. Christine Schimmel, in the Department of Counseling, Rehabilitation Counseling, and Counseling Psychology at West Virginia University at (304) 2932266, or by e-mail at chris.schimmel@mail.wvu.edu.

Sincerely,

Courtney Hull, M.S.

cnh0008@mix.wvu.edu

(812) 251-8980 


\section{Appendix M: SSEIT Permission Email}

Nicola Schutte

(e) 8:48 PM (0 minutes ago)

to me

Thank you for your message. You are welcome to use the Assessing Emotions (SSEIT) scale. Please find attached the manuscript version of a published chapter that contains the scale and background information, including regarding scoring, reliability and validity. Kind regards, Nicola Schutte

...

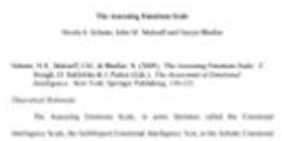

Assessing Emotion... 
Appendix N: Curriculum Vitae

\section{Courtney N. Hull, M.S.}

241 Kennedy Dr. Crowley, TX. 76036

(812) 251-8980

Cnh0008@mix.wvu.edu

\section{Education}

\section{West Virginia University}

502 Allen Hall, Morgantown, WV. 26506

08/2016-Present (Anticipated 05/2021)

Degree: Ph.D.

Program: Counseling Psychology

Accreditation: American Psychological Association

Dissertation: Correctional Officer Attitudes toward Offenders with Mental Illness: The

Relationships between Attitudes, Burnout, Emotional Intelligence, and Mental Health

Training

Indiana State University

200 N. $7^{\text {th }}$ St. Terre Haute, IN. 47809

06/2014-05/2016

Degree: M.S.

Program: Clinical Mental Health Counseling

Accreditation: CACREP

Indiana State University

200 N. $7^{\text {th }}$ St. Terre Haute, IN. 47809

08/2010-05/2014

Degree: B.S.

Major: Psychology

Minor: Human Development and Family Studies

Certificate: Gerontology

\section{Supervised Clinical Experience}

Doctoral Practicum Student

(08/2019-Present)

Federal Correctional Complex, Hazelton

United States Penitentiary and Secure Female Facility

1640 Sky View Dr., Bruceton Mills, WV 26525

- Provide routine and emergent therapy services to CARE1-MH, CARE2-MH, and

CARE3-MH inmates

- Facilitate Basic Cognitive Skills and Criminal Thinking groups

- Complete initial intake screenings and transfer intake screenings

- Co-conduct SFF Special Housing Unit rounds

- Conduct Suicide Risk Assessments, Risk of Sexual Victimization Assessments, and Risk of Sexual Abusiveness Assessments 
- Co-facilitate Suicide Companion Trainings

- Responsible for the completion and maintenance of mental health documentation and records

- Supervisors: Jason Smith, Psy.D.; Alexandra Crouch, Psy.D.

Doctoral Practicum Student

$(08 / 2018-08 / 2019)$

Federal Correctional Institution, Morgantown

446 Green Bag Rd., Morgantown, WV 26501

- Completed initial intake screenings and transfer intake screenings

- Facilitated Cognitive Processing Therapy for Trauma, Basic Cognitive Skills, Acceptance and Commitment Therapy Mindfulness, Dialectical Behavior Therapy, and Residential Drug Abuse Program process groups

- Conducted individual therapy sessions with CARE1-MH and CARE2-MH inmates

- Developed and implemented inmate suicide companion cadre trainings on aging in prison and opioid use

- Administered, scored, and interpreted standardized psychological assessment measures, and developed integrated reports

- Responsible for the completion and maintenance of mental health documentation and records

- Supervisor: Abby McCabe, Ph.D.

\section{Doctoral Practicum Student}

Fremouw, Sigley, and Baker Psychological Associates

$(05 / 2017-08 / 2019)$

1224 Pineview Dr., Morgantown, WV 26505

- Conducted competency and criminal responsibility, parental fitness, and Social Security Administration evaluations

- Administered, scored, and interpreted standardized psychological assessment measures

- Completed comprehensive bio-psycho-social clinical interviews

- Developed integrated reports

- Responsible for the completion and maintenance of mental health documentation and records

- Supervisor: Edward Baker, Ph.D.

Doctoral Practicum Student

United Summit Center

6 Hospital Dr., Clarksburg, WV 26301

- Conducted individual psychotherapy sessions

- Implemented drug and alcohol assessments

- Administered, scored, and interpreted standardized psychological assessment measures, and developed integrated reports

- Conducted comprehensive bio-psycho-social clinical interviews

- Responsible for the completion and maintenance of mental health documentation and records

- Supervisor: Dennis Kojsza, Ph.D.

Masters Student Intern

Wabash Valley Correctional Facility

$(08 / 2015-05 / 2016)$ 
6908 S. Old U.S. Highway 41, Carlisle, IN 47838

- Conducted individual and group counseling sessions with offenders at maximum security facility

- Conducted clinical assessments and developed diagnostic impressions

- Provided supervised crisis intervention services

- Responsible for the completion and maintenance of mental health documentation and records

- 378 direct hours and 904 total clinical internship hours completed

- Supervisors: Bradley Huffey, Ph.D., HSPP; Lisa Isberg, Psy.D.

\section{Masters Practicum Student}

William and Norma Grosjean Clinic at University Hall

$(01 / 2015-12 / 2015)$ 200 N. $7^{\text {th }}$ St., Terre Haute, IN 47809

- Conducted individual, family, couple, and group counseling sessions

- Responsible for the completion and maintenance of client documentation and records

- 74 direct hours and 278 total clinical practicum hours completed

- Supervisors (Live Supervision): Bridget Roberts-Pittman, Ph.D., HSPP, LMFT; Anna Viviani, Ph.D., LMHC; Catherine Tucker, Ph.D., LMHC

\section{Undergraduate Intern}

$(05 / 2013-08 / 2013)$

\section{Harsha Behavioral Center, Geriatric Unit}

1980 E. Woodsmall Dr., Terre Haute, IN 47802

- Monitored geriatric patients with various clinical diagnoses

- Facilitated group memory recall activities

- Collaborated with Recreational Therapist to design therapy templates and assisted in compiling daily participation records

- Supervisor: Christine Watson, LCSW

\section{Publications}

Tucker, C., Schieffer, K., Wills, T. J., Hull, C., \& Murphy, Q. (2017). Enhancing socialemotional skills in at-risk preschool students through Theraplay based groups: The sunshine circle model. International Journal of Play Therapy, 26(4), 185-195.

doi: $10.1037 / \mathrm{pla} 0000054$

\section{Research Presentations}

\section{International Presentations}

Daniels, J. A., Allen, T., Hull, C., Kinder, A., Noble, G., Bridendolph, N., Fanning, S., Kepner, C., \& Nauser, J. (2018, June). Qualitative data meets text analytics: Analyses of kidnapper interview transcripts. Poster presentation at the 2018 International Congress of Applied Psychology. Montreal, Quebec, Canada.

Gardner, L. \& Hull, C. (2016, February). Trauma informed care in conflict areas. Poster presentation at the International Conference on the Impact of Armed Conflict and Terrorism on Children. Nairobi, Kenya. 
Boink, C., Gardner, L., Hull, C. \& Latke, R. (2014, December). Trauma informed care: U.S. perspectives. Presentation at the Art and Expressive Therapies: Indian and U.S. Perspectives Conference. Nagindas Khandwal College, Mumbai, India.

\section{Regional Presentations}

Hull, C. (2019, April). Aging in prison: Health outcomes and provision of care. Poster presentation at the Great Lakes Regional Counseling Psychology Conference. Louisville, KY.

Noble, G. \& Hull, C. (2019, April). An exploratory study of forensic factors affecting parental fitness. Poster presentation at the Great Lakes Regional Counseling Psychology Conference. Louisville, KY.

Hull, C. \& Noble, G. (2018, April). Emotional intelligence and prosocial behavior in adolescents. Poster presentation at the Great Lakes Regional Counseling Psychology Conference. Kalamazoo, MI.

Kinder, A. \& Hull, C. (2018, April). Social comparison in the digital age. Poster presentation at the Great Lakes Regional Counseling Psychology Conference. Kalamazoo, MI.

Thomas, K. \& Hull, C. (2018, April). Motivational interviewing for decreasing maladaptive health behaviors. Poster presentation at the Great Lakes Regional Counseling Psychology Conference. Kalamazoo, MI.

Daniels, J., Bridendolph, N., Hull, C., \& Kinder, A. (2017, August). Counseling psychology areas of emphasis: A survey of APA approved doctoral programs. Poster presentation at the Annual Convention of the American Psychological Association. Washington, D.C.

Latorre, C., Milam, S., Hull, C., \& Kinder, A. (2017, April). Get in on the action: Promoting social justice on campus. Roundtable presentation at the Great Lakes Regional Counseling Psychology Conference. Muncie, IN.

Hull, C., Kinder, A., \& Thomas, K. (2017, April). Multicultural and recruitment issues in dementia caregiver studies. Poster presentation at the Great Lakes Regional Counseling Psychology Conference. Muncie, IN.

Hull, C. \& Kruger, T. (2017, March). Recruiting dementia caregivers for a stress study: Lessons learned through a master's research project. Paper presentation at the Association for Gerontology in Higher Education's 43 ${ }^{\text {rd }}$ Annual Meeting and Educational Leadership Conference. Miami, FL.

Hull, C. \& Kruger, T. (2016, November). Understanding dementia: The effects of supportive services on the perceived stress of caregivers. Poster presentation at The Gerontological Society of America's 69th Annual Scientific Meeting. New Orleans, LA.

Murphy, Q. \& Hull, C. (2015, October). The forgotten, unwanted: The experiences of children of color in the U.S. foster care system. Poster presentation at the 2015 Indiana State University Diversity Symposium. Terre Haute, IN. 


\section{Grants}

\begin{tabular}{|l|l|l|l|}
\hline Grant & Status & Date Received & Amount \\
\hline Robert E. Stitzel Graduate & Approved & $05 / 01 / 2019$ & $\$ 1,200$ \\
\hline $\begin{array}{l}\text { Bayh College of Education } \\
\text { Graduate Student Research and } \\
\text { Development Fund }\end{array}$ & Approved & $12 / 07 / 2015$ & $\$ 300$ \\
\hline $\begin{array}{l}\text { Indiana State University } \\
\text { Graduate and Professional } \\
\text { Studies Student Research Fund }\end{array}$ & Approved & $10 / 20 / 2015$ & \\
\hline
\end{tabular}

\section{Supervised Teaching Experience}

Graduate Instructor, COUN 303 Helping Professions

(08/2019-Present)

West Virginia University, Department of Counseling, Rehabilitation

Counseling, and Counseling Psychology

355 Oakland St., Morgantown, WV 26506

- Implement class curriculum related to helping professions course

- Utilize online e-campus program to monitor student progress and maintain student grades

- Ensure timely and effective communication with students

- Supervisor: Regina Burgess, M.S.

University 101 Instructor

$(08 / 2015-12 / 2015)$

Indiana State University, University College

200 N. $7^{\text {th }}$ St., Terre Haute, IN 47809

- Developed and implemented class syllabus and curriculum related to student adjustment to the university setting

- Monitored student progress and maintained student grades through online Blackboard program

- Managed classroom duties and initiated a collaborative atmosphere of education

- Supervisor: Roberta Allen, M.A.

Sycamore Success Instructor

Indiana State University, Learn, Engage, Achieve, Persist (LEAP) Program

$(07 / 2015)$

200 N. $7^{\text {th }}$ St., Terre Haute, IN 47809 
- Led student success classes on topics including time management, goal setting, communication, university resources, motivation, and growth mindset

- Upheld program standards of attendance, personal conduct, and participation

- Supervisor: Roberta Allen, M.A.

Teaching Assistant, AHS 305 Society and Aging

$(09 / 2013-05 / 2014)$

Indiana State University, Applied Health Sciences Department

200 N. $7^{\text {th }}$ St., Terre Haute, IN 47809

- Provided administrative support for exam, discussion, and writing assignments through grading and maintaining grading records

- Managed and responded to course-related e-mail

- Prepared and maintained web page for course materials

- Supervisor: Tina Kruger, Ph.D.

\section{Clinical Employment}

Mental Health Specialist

\section{Chestnut Ridge Behavioral Health Center}

$(03 / 2017-09 / 2017)$

J. W. Ruby Memorial Hospital

930 Chestnut Ridge Rd., Morgantown, WV 26505

- Facilitated therapeutic groups maintained a therapeutic milieu

- Collaborated with members of the interdisciplinary team

- Utilized master treatment plans for assistance in identifying and implementing developmental age appropriate interventions with patients

- Assisted with therapeutic intervention, including verbal de-escalation and physical intervention

- Implemented appropriate interventions and documented patient's responses in the medical record using the DAR format under the supervision of a registered nurse

Admission Coordinator

$(07 / 2014-07 / 2016)$

\section{Harsha Behavioral Center}

1980 E. Woodsmall Dr., Terre Haute, IN 47802

- Interviewed patients and legal guardians to obtain eligibility information for admission

- Conducted intake assessments and pre-admission screenings

- Gathered and processed admission data in a timely fashion

- Entered patient information and data into electronic medical records

- Demonstrated the ability to be organized and flexible in emergency situations

\section{Mental Health Technician}

$(08 / 2013-07 / 2014)$

Harsha Behavioral Center

1980 E. Woodsmall Dr., Terre Haute, IN 47802

- Observed and recorded patients' compliance, behavior, mental status, and activities, inclusive of various populations and disorders

- Administered guidance in activities of daily living and in de-escalation techniques

- Performed and recorded patient vital signs, intake, output and provided general nursing care 
- Supervised patients in group, individual, and recreational therapies under the direction of program therapists

\section{Academic Mentor}

\section{Indiana State University Academic Opportunity Program}

$(08 / 2012-05 / 2014)$

200 N. $7^{\text {th }}$ St., Terre Haute, IN 47809

- Facilitated the development of students' time management skills

- Assessed students' tutoring needs

- Monitored and recorded academic progress of students

- Assisted in developing a sense of belonging and campus connection for students

\section{Leadership}

Campus Representative

(09/2018-Present)

American Psychology-Law Society

American Psychological Association, Division 41

Student Representative

(05/2017-Present)

Diversity Recruitment and Retention Committee

West Virginia University Department of Counseling,

Rehabilitation Counseling, and Counseling Psychology

Speak Out Reach Out

(08/2016-Present)

- Co-President (2017-2018)

- Treasurer (2016-2017)

Chi Sigma Iota International Honor Society

$(03 / 2015-05 / 2016)$

- Co-President, Iota Sigma Tau (2015- 2016)

Alpha Omicron Pi International Fraternity

- Alumna Advisor, Vice President of Standards (2016-2017)

(08/2010-Present)

- Alumna Advisor, Vice President of Education (2015-2016)

- Chapter President (2013-2014)

- Indiana State University President's Cup 2014, Student Organization of the Year 2013, Indiana State University President's Cup 2013

- New Member Educator (2012-2013)

- $\quad$ Keeper of Ritual (2011-2012)

- 200 hours of community service completed (2010-2014)

\section{Volunteer Work}

Appalachian Prison Book Project

(08/2018-Present)

- Office support assistant

- Mail committee member

Osher Life Long Learning Institute at West Virginia University

$(08 / 2018-08 / 2019)$

- Office assistant and event organizer

Alzheimer's Association, West Virginia Chapter

$(08 / 2016-12 / 2018)$

- Office assistant and community workshop presenter 
- Caregiver support group co-facilitator

- Walk to End Alzheimer's volunteer and team captain

LeadWELL Peer Mentorship Program

$(09 / 2017-05 / 2018)$

- Graduate mentor

- Communication and leadership trainer

Hearts of Gold Service Dog Training Center

$(05 / 2017-08 / 2017)$

- Office assistant

- Community workshop presenter

Maple Center for Integrative Health

$(01 / 2015-01 / 2016)$

- Social media liaison

Annual Convention of the American Psychological Association

$(08 / 2015)$

Toronto, Canada

- ADA Disabilities Center Volunteer

- Assisted attendees with transportation needs, hearing impairment services, and navigation assistance

\section{Professional Affiliations}

American Psychological Association (07/2015-Present)

- Division 17 member, Society of Counseling Psychology

- Division 41 member, American Psychology-Law Society

Psi Chi Academic Fraternity (10/2012-Present)

\section{Awards}

West Virginia University Three Year Provost Fellowship

$(08 / 2016-08 / 2019)$

- 250 community service hours completed

College of Education and Human Services Scholarship

West Virginia University

Alzheimer's Association Early Investigator's Scholarship

Graduate Assistant

$(07 / 2014-05 / 2016)$

Indiana State University Center for Student Success

200 N. $7^{\text {th }}$ St., Terre Haute, IN 47809

- Supervised tutorial and supplemental instruction service employees

- Led one-on-one academic achievement sessions for psychology and counseling courses

- Created weekly tutor/student meeting schedules

- Maintained weekly time logs of employees

- Documented student session statistics

Graduate Assistant

William and Norma Grosjean Clinic at University Hall

$(05 / 2015-07 / 2015)$

401 North 7th Street, Terre Haute, IN 47809 
- Organized professional communications

- Ensured clinic productivity by maintaining calendars and scheduling patient appointments

- Kept office equipment operational by following operating instructions and maintaining supplies

Dr. Michele C. Boyer Professional Development Award

Sycamore Leadership Awards

- Senior leadership Award Recipient

\section{International Experiences}

Indiana State University Faculty-led Study Abroad

$(02 / 2016)$

Nairobi, Kenya; Nakuru, Kenya

- Collaborated with local organizations to address childhood education and childhood poverty

- Facilitated volunteerism workshop with university students

- Presented on trauma informed response to international conflict.

- Supervisor: Catherine Tucker, Ph.D., LMHC

Indiana State University Faculty-led Study Abroad

Mumbai, India; New Delhi, India; Jaipur, India; Agra, India

- Travel in conjunction with COUN 631

- Collaborated with local organizations to address childhood sexual abuse, and childhood poverty in India

- Presented on U.S. perspectives on trauma informed care at the Art and Expressive Therapies: Indian and U.S. Perspectives Conference at Nagindas Khandwal College in Mumbai, India.

- Supervisor: Catherine Tucker, Ph.D., LMHC

Indiana State University Faculty-led Study Abroad

$(07 / 2012-08 / 2012)$

London, England

- Travel in conjunction with PSY 485 and PSY 032

- Participated in group and independent exploration of English perspectives on consciousness, perception, and British counterculture.

- Supervisor: Tom Johnson, Ph.D. 